\title{
Electromagnetic Probes at RHIC-II
}

\author{
G. David ${ }^{a}$, R. Rapp ${ }^{b}$ and Z. Xu ${ }^{a}$ \\ a Physics Department, Brookhaven National Laboratory, Upton, NY 11793-5000, \\ USA \\ ${ }^{\mathrm{b}}$ Cyclotron Institute and Physics Department, Texas A\&M University, College \\ Station, TX 77843-3366, USA
}

\begin{abstract}
We summarize how future measurements of electromagnetic (EM) probes at the Relativistic Heavy Ion Collider (RHIC), in connection with theoretical analysis, can advance our understanding of strongly interacting matter at high energy densities and temperatures. After a brief survey of the important role that EM probes data have played at the Super Proton Synchrotron (SPS, CERN) and RHIC to date, we identify key physics objectives and observables that remain to be addressed to characterize the (strongly interacting) Quark-Gluon Plasma (sQGP) and associated transition properties at RHIC. These include medium modifications of vector mesons via low-mass dileptons, a temperature measurement of the hot phases via continuum radiation, as well as $\gamma-\gamma$ correlations to characterize early source sizes. We outline strategies to establish microscopic matter and transition properties such as the number of degrees of freedom in the sQGP, the origin of hadron masses and manifestations of chiral symmetry restoration, which will require accompanying but rather well-defined advances in theory. Increased experimental precision, an order of magnitude higher statistics than currently achievable, as well as a detailed scan of colliding species and energies are mandatory to discriminate between theoretical interpretations. This increased precision can be achieved through hardware upgrades to the large RHIC detectors (PHENIX and STAR) along with at least a factor of ten increase in luminosity over the next few years, as envisioned for RHIC-II.
\end{abstract}

Key words: direct photon, isolated photon, quark-gluon plasma, relativistic heavy ion collision

PACS: 25.75.-q, 25.75.Nq, 12.38.Mh, 13.85.Qk

Preprint submitted to Elsevier Science 11 July 2018 


\section{Contents}

1 INTRODUCTION

1.1 Toward Discovery and Characterization of Hot and Dense QCD Matter

1.2 Where EM Probes Are Unique or Very Important

1.3 Theory vs Experiment at the CERN-SPS 8

2 THEORETICAL CONSIDERATIONS AND PREDICTIONS

2.1 Objectives and Framework

2.2 QCD Lattice Results 13

2.3 Sum Rules 14

2.4 Temperature and Degrees of Freedom 18

2.5 In-Medium Spectral Functions below and above $T_{c}$

2.6 Low-Mass Dilepton Spectra 29

2.7 Chiral Symmetry Restoration 30

2.8 Electromagnetic Signatures of the Color Glass Condensate 35

3 OBSERVABLES: STATE OF THE ART 40

3.1 Low-Mass Dileptons 40

3.2 The $\phi(1020)$ : Hadronic vs Leptonic Decays and $v_{2}$

3.3 Direct Photon Spectra 43

3.4 Direct Photon $R_{A A}$

3.5 Photon Azimuthal Asymmetries (Elliptic Flow) 47

3.6 Electron $R_{A A}$ and Flow 47

3.7 Direct Thermal Photons via Low-Mass Dielectrons 49

4 THE FUTURE

4.1 Detector upgrades

4.2 New Measurements 
4.3 High Statistics and Energy/Species Scan 59

4.4 From Observables to Pertinent Theoretical Issues 62

5 SUMMARY AND CONCLUDING REMARKS 63

References 


\section{INTRODUCTION}

\subsection{Toward Discovery and Characterization of Hot and Dense QCD Matter}

One of the key goals of ultrarelativistic heavy-ion collisions is the creation of hot and dense strongly interacting matter that: (i) resembles the conditions in the early universe; (ii) can be related to the phase diagram of the underlying theory (Quantum Chromodynamics (QCD)); and (iii) enables the discovery of new phases. Recent surveys of results from the first three years of data taking by the four experiments at the Relativistic Heavy Ion Collider (RHIC) $[1,2,3,4]$ are unanimous in their conclusion that a new form of matter has been created. This matter is very dense, opaque and exhibits a high level of collectivity which has largely been attributed to the expansion of a partonic phase. It is inconsistent with naive expectations based on a weakly-interacting (gas-like) Quark-Gluon Plasma (wQGP), while it is best described in terms of a socalled strongly interacting QGP (sQGP) constituting an almost perfect fluid. Thus, a discovery has been made in a qualitative sense, but the properties of this new state of matter remain under intense debate.

A closer look indeed reveals that we are still quite far from a coherent and quantitative description of the sQGP at RHIC. The wealth and precision of new data from Run-4 and Run-5 [5] is a first step in this direction. Measurements of previously inaccessible signals, such as semileptonic electron-decay spectra, $J / \psi$ production and three-hadron correlations, and improvements on the statistical and systematic errors, as well as the range of the existing data, have been achieved. In addition, new and ongoing analyses of SPS data, most notably from NA60 [6,7] (including low- and intermediate-mass dileptons and $J / \psi$ production), have reached unprecedented levels of precision that now can distinguish between model predictions which were consistent with earlier data sets. Furthermore, issues have been raised again, that seemed to have essentially been settled a few years ago, e.g. the energy-loss mechanism of jet quenching. It therefore appears fair to say that whereas the existence of the new form of matter has been established, we neither understand its microscopic properties nor deduced from it convincing signals of the phase transition itself. While theory has also made substantial progress in the last few years by moving from more signal-specific explanations to a coherent description by connecting different phenomena and improving theoretical tools, a widely accepted "grand scheme" encompassing both bulk and microscopic components has not yet been realized. As ideas get refined (largely steered by data), different theoretical predictions often approach each other, thus increasing the demand for higher quality measurements to differentiate between them. 


\begin{tabular}{|l|cccc|cc|c|}
\hline Upgrades & High $T$ & QCD $\ldots$ & QGP & Spin & Low- $x$ \\
\hline & $e+e-$ & $\begin{array}{c}\text { heavy } \\
\text { flavor }\end{array}$ & $\begin{array}{c}\text { jet } \\
\text { tomog. }\end{array}$ & quarkonia & $\mathrm{W}$ & $\Delta G / G$ & \\
\hline PHENIX & & & & & & & \\
\hline Hadron blind detector & $\mathrm{X}$ & & & & & & \\
Vertex Tracker & $\mathrm{X}$ & $\mathrm{X}$ & $\mathrm{O}$ & $\mathrm{O}$ & & $\mathrm{X}$ & $\mathrm{O}$ \\
Muon Trigger & & & & $\mathrm{O}$ & $\mathrm{X}$ & & \\
Forward Cal (NCC) & & & $\mathrm{O}$ & $\mathrm{O}$ & $\mathrm{O}$ & & $\mathrm{X}$ \\
\hline STAR & & & & & & & \\
\hline Time of Flight (ToF) & $\mathrm{X}$ & $\mathrm{O}$ & $\mathrm{X}$ & $\mathrm{X}$ & & & \\
Heavy Flavor Tracker & $\mathrm{X}$ & $\mathrm{X}$ & & $\mathrm{X}$ & & & \\
Forward Tracker & & $\mathrm{O}$ & & & $\mathrm{X}$ & $\mathrm{O}$ & \\
Forward Cal (FMS) & & & & & & $\mathrm{O}$ & $\mathrm{X}$ \\
DAQ 1000 & $\mathrm{O}$ & $\mathrm{O}$ & $\mathrm{X}$ & $\mathrm{X}$ & $\mathrm{O}$ & $\mathrm{O}$ & $\mathrm{O}$ \\
\hline RHIC Luminosity & $\mathrm{O}$ & $\mathrm{O}$ & $\mathrm{X}$ & $\mathrm{X}$ & $\mathrm{O}$ & $\mathrm{O}$ & $\mathrm{O}$ \\
\hline
\end{tabular}

Table 1

Matrix of detector or accelerator upgrades vs physics measurements. $\mathrm{X}=$ upgrade critical for measurement. $\mathrm{O}=$ upgrade important for measurement.

After very successful operation of RHIC 1 and its detectors over the first five years of data taking, we are approaching a point where further progress requires improved experimental capabilities. Table 1 lists important physics topics that are either beyond our current reach or would be significantly enhanced with the indicated detector and luminosity upgrades. These should be put into context with overarching questions in the investigation of the sQGP and chi$\mathrm{ral} /$ deconfinement transitions, emphasizing those for which electromagnetic probes are particularly relevant, including:

- What are the temperatures and corresponding system sizes of thermalized matter at its early stages?

- How do hadron properties change in hot and dense matter and how are

$\overline{1}$ By 2004 (Run-4) the accelerator exceeded design luminosities by a factor of 2.5 both in $\mathrm{Au}+\mathrm{Au}$ and $p p$ collisions. RHIC collided 4 different systems including asymmetric $\mathrm{d}+\mathrm{Au}$ interactions, collisions at 6 different center-of-mass energies, among others at $19 \mathrm{GeV}(\mathrm{Au}+\mathrm{Au})$ and $22 \mathrm{GeV}(\mathrm{Cu}+\mathrm{Cu})$, establishing the first overlap with the CERN SPS. In 2007 (Run-7) it delivered $3260 \mu \mathrm{b}^{-1}$ integrated $\mathrm{Au}+\mathrm{Au}$ luminosity at $200 \mathrm{GeV}$, in 2008 (Run-5) the first tests with $9 \mathrm{GeV}$ and $5 \mathrm{GeV}$ per nucleon (below injection energy) are planned with $\mathrm{Au}+\mathrm{Au}$. 

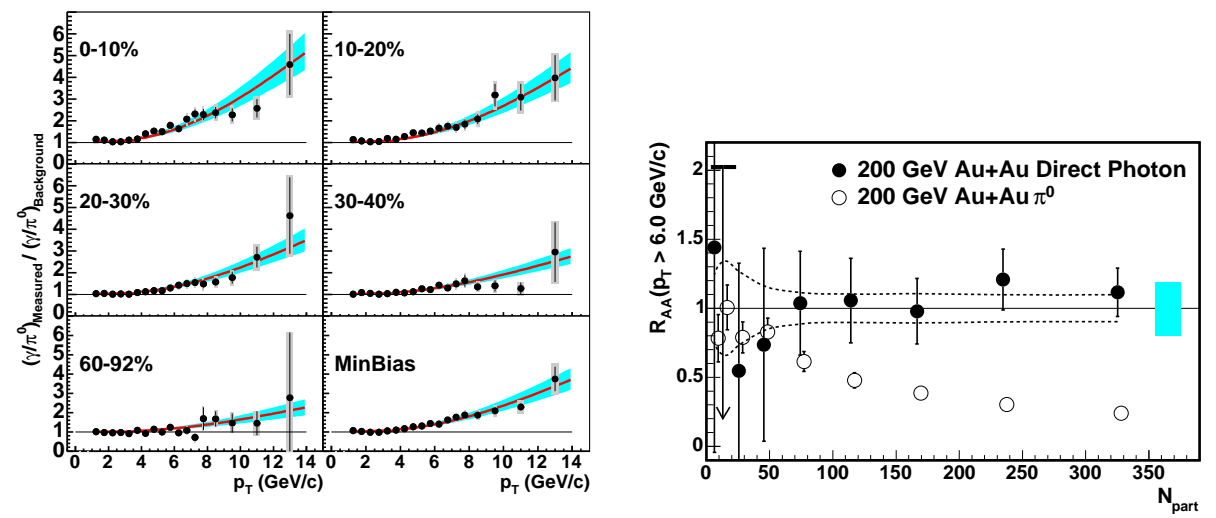

Fig. 1. Left panel: direct photon excess ratios measured by PHENIX in $\sqrt{s_{N N}}=200$ $\mathrm{GeV} \mathrm{Au+Au} \mathrm{collisions} \mathrm{[8].} \mathrm{The} \mathrm{curves} \mathrm{are} \mathrm{the} \mathrm{ratios} \mathrm{predicted} \mathrm{by} \mathrm{NLO} \mathrm{pQCD} \mathrm{[9].}$ Right panel: integrated $R_{A A}$ for photons and $\pi^{0} \mathrm{~s}$ as a function of the number of participants, $N_{\text {part }}$, measured by PHENIX in $\sqrt{s_{N N}}=200 \mathrm{GeV} \mathrm{Au}+\mathrm{Au}$ collisions [8].

hadron masses generated? How do the medium modifications depend on temperature and net/total baryon density?

- Can we deduce signatures of Chiral Symmetry Restoration $(\chi \mathrm{SR})$ and, if so, how is it realized?

- What are the relevant degrees of freedom in the sQGP? Does it harbor bound states and/or resonances?

- How do the medium properties change if the net baryon density is increased? Can a QCD critical point be found?

- How does the system reach (local) equilibrium on the apparently short time scales required by hydrodynamics?

- What exactly causes jet quenching?

In the remainder of this introductory section, we will first elaborate in somewhat more detail on the features and strongholds of EM probes in heavy-ion collisions (Sec. 1.2). In particular, we will discuss the achievements and shortcomings of the EM probes program at the SPS (Sec. 1.3) which will help us to sharpen the case for future RHIC measurements. In Sec. 2 we lay out the theoretical framework for describing and interpreting EM probes. We will also provide predictions for observables and formulate strategies for deducing information on key properties of the medium that may not follow from an immediate interpretation of experimental data. In Sec. 3 we give a brief overview on the current status of EM observables at RHIC. In Sec. 4 we then focus on how future detector and accelerator upgrades can be used and geared toward answering the above questions. In Sec. 5 we reiterate the main points of this document. 


\subsection{Where EM Probes Are Unique or Very Important}

Electromagnetic probes are real, $\gamma$, and virtual photons (dileptons), $\gamma^{*} \rightarrow l^{+} l^{-}$ where $l=e$ and $\mu$. They are emitted from the entire reaction volume throughout the evolution of a heavy-ion collision, from first impact in primordial (hard) $N N$ collisions until decays of long-lived hadrons long after strong interactions have ceased. Once created, most of them leave the interaction volume unchanged due to their negligible final-state interaction with the stronglyinteracting medium. Following the usual terminology we define direct photons as those that are not decay products of final state hadrons. Accordingly, direct photon spectra are defined as the spectra remaining after subtraction/elimination 2 of photons coming from final-state decays ("background" or "decay photons"), whereas in measured dilepton spectra pairs from final state decays are often included and their estimated contribution shown separately ("hadronic decay cocktail"). The broad category of "direct" photons is then often subdivided according to their source such as "prompt" photons (from hard scattering) "thermal" photons, "jet conversion" photons, etc.

Unfortunately, the same property that allows photons to escape, $\alpha<<\alpha_{s}$, also leads to major experimental challenges: low rates and large backgrounds from the above-mentioned late hadron decays (e.g. Dalitz-decays for dileptons and $\pi^{0} \rightarrow \gamma \gamma, \eta \rightarrow \gamma \gamma$, etc. for real photons).

The fact that direct photons from initial hard scatterings escape the system [8] is demonstrated in Fig. 1 where the measured direct photon excess ratid 3 at sufficiently high transverse momentum, $p_{T}$, is shown to be consistent with next-to-leading order (NLO) perturbative $\mathrm{QCD}$ (pQCD) calculations, even in the most central $\mathrm{Au}+\mathrm{Au}$ collisions.

The interesting physics we are primarily concerned with here is at significantly lower $p_{T}$ and dilepton invariant masses, $M$ than inferred from Fig. 1. Thus, in this review, we stress the importance of precision data at masses and momenta below $\sim 3-4 \mathrm{GeV}$.

Electromagnetic probes are unique.

- They give direct access to the in-medium modifications of hadronic states (the $\rho(770), \omega(782)$, and $\phi(1020)$ vector mesons) via dilepton invariant-mass spectra, which can illuminate the nature of hadron mass generation and thus the origin of $\sim 98 \%$ of the visible mass in the universe as well as related changes in the structure of the QCD vacuum, including chiral symmetry

$\overline{2}$ This can be done in a number of ways like statistical subtraction, "tagging", etc.

3 The ratio of inclusive photons over hadron decay photons. For more details on the direct photon excess ratios and $R_{A A}$ see Sec 3.4. 
restoration. Detecting novel nonperturbative (resonant) correlations in the sQGP at masses above $\sim 1 \mathrm{GeV}$ is also possible.

- They can be used to infer the temperature of the system during its hottest phases via direct thermal photon and dilepton radiation. In addition, HBT interferometry of thermal photons offers the cleanest measurement of early system sizes.

Indirect consequences of the above studies include insights into the mechanism of rapid thermalization, e.g. via resonance formation in the sQGP. The effective degrees of freedom can be determined if a temperature measurement is complemented with independent information on the energy or entropy density. Although not unique, EM probes are valuable for (i) disentangling the energy loss mechanism of jets (jet quenching) by inferring photon radiation off energetic quarks and establishing the jet energy scale in $\gamma$-hadron jets and (ii) obtaining complementary information on (early) matter flow by investigating photon elliptic flow, $v_{2}$, which, in turn, discriminates between thermal photons and those radiated off jets.

In the following sections, we will elaborate on these statements together with the requirements to measure associated observables with sufficient accuracy.

\subsection{Theory vs Experiment at the CERN-SPS}

Before beginning a more detailed discussion of the theoretical aspects underlying a comprehensive electromagnetic probes program for future RHIC runs, we briefly review the main achievements at the SPS prior to the new NA60 data [6,7], discussed later. An example of one theoretical approach to electromagnetic emission simultaneously applied to semi-/central $\mathrm{Pb}+\mathrm{Au}$ and $\mathrm{Pb}+\mathrm{Pb}$ data is compiled in Figs. 2 and 3. The data suggest a common thermal source with an initial temperature of $T_{0} \simeq 210 \pm 30 \mathrm{MeV}$ and a lifetime of about $12 \pm 3 \mathrm{fm} / c$ with a thermal freezeout temperature of around $100-120 \mathrm{MeV}$. Importantly, the low-mass dilepton enhancement in the CERES $e^{+} e^{-}$data [10] (upper panel of Fig. 2) requires substantial medium effects over a sufficiently long lifetime, on the $\rho$ spectral function. However, decisive discrimination between a dropping-mass scenario [11] and a substantially broadened spectral function [12] was not possible since the QGP contribution is small, around $10-15 \%$. The sensitivity to QGP radiation increases in the intermediatemass dimuon spectra of NA50 [13] (lower panel) where the observed factor of two excess over the baseline charm and Drell-Yan sources can be reasonably accommodated with thermal radiation containing a $30-50 \%[14,15,16]$ QGP component, the main evidence for the $T_{0}$ quoted above. A very similar decomposition is found in the WA98 direct photon spectra [17] at $q_{t} \simeq 2 \mathrm{GeV}$ (upper panel of Fig. 3) where $q$ is the real or virtual photon momentum. The 

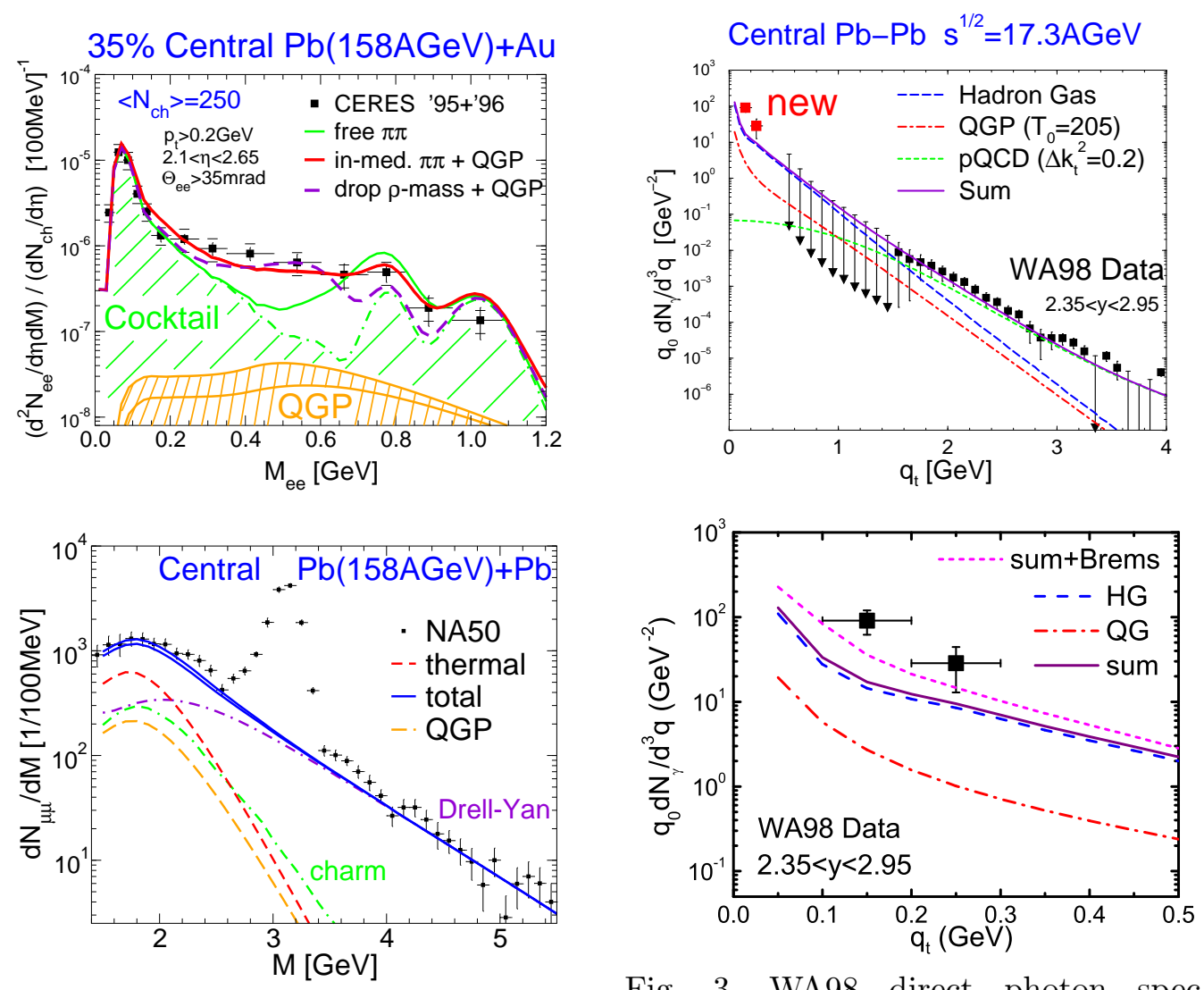

Fig. 2. Dilepton spectra at the SPS compared to calculations $[12,14]$ within an expanding thermal fireball model. Upper panel: CERES/NA45 low-mass dielectrons [10]; lower panel: NA50 intermediate-mass dimuons [13].

Fig. 3. WA98 direct photon spectra $[17,18]$ compared to thermal emission within the same fireball model as in Fig. 2 with the same EM correlator used for dileptons extrapolated to the photon point $[19,21]$. The lower panel expands the low $q_{t}$ region.

recently published low-momentum data [18] (lower panel), extracted via photon HBT methods, are not easily reconciled with theory $[19,20]$. The inclusion of soft Bremsstrahlung off $\pi \pi$ and $\pi K$ scattering appears to improve the situation [21]. The low-momentum yield is essentially emanating from the later stages of the fireball evolution and thus it is proportional to the total fireball lifetime.

The SPS electromagnetic probe program was incomplete for a number of reasons ranging from insufficient statistics to draw conclusions to the lack of experimental cross checks. A partial list of open questions is given below.

(a) There was no decisive discrimination of in-medium $\rho$ modifications (although the recent NA60 data [6,7] has improved the situation, albeit in a smaller system, see below). In particular, no systematic excitation function was obtained through an energy scan. The only CERES/NA45 low-energy run at $E_{\text {lab }}=40 A \mathrm{GeV}[22]$ indicated an increase over the enhancement at 


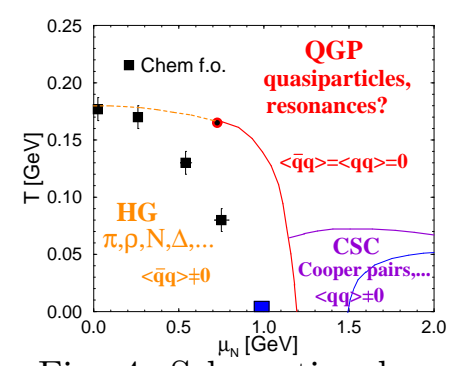

Fig. 4. Schematic phase diagram of QCD including temperature "measurements" from chemical analysis of produced hadrons [25].

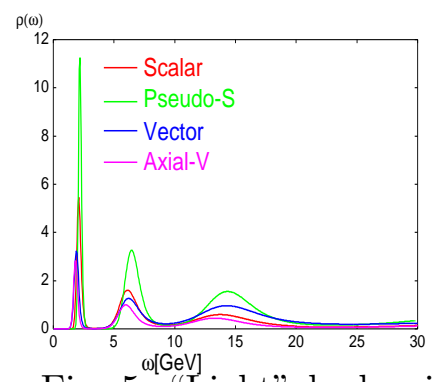

Fig. 5. "Light" hadronic spectral functions in the QGP $\left(T=1.4 T_{c}\right)$ from lattice QCD [34], exhibiting resonances with approximate chiral symmetry.

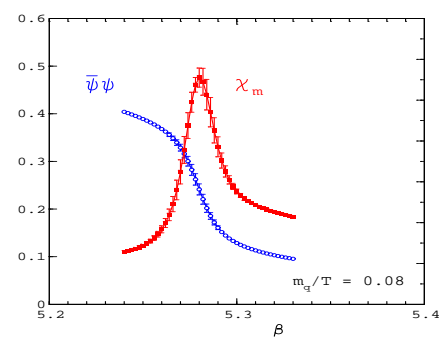

Fig. 6. Temperature dependence of the quark chiral condensate, $\langle\bar{q} q\rangle(T)$, computed in unquenched lattice QCD [41].

$160 A \mathrm{GeV}$ but with large errors.

(b) The Cronin enhancement in the primordial pQCD photons, essential for an accurate assessment of the thermal photon yield in the $q_{t} \simeq 2 \mathrm{GeV}$ region, was not determined.

(c) Charm dileptons were not explicitly identified, hampering the temperature extraction in the intermediate-mass dimuon spectra. However, NA60 made substantial progress in an intermediate-size system [23].

(d) There was no experimental redundancy to cross-check measurements and create competition.

(e) The QGP contribution to EM probes at the SPS is perhaps too modest in principle to reveal itself in a significant way.

(f) The level of early thermalization at the SPS may be insufficient to justify thermal approaches in the early phases, as may be indicated by the lack of elliptic flow in hadron data relative to hydrodynamic models above $p_{T} \sim$ $1 \mathrm{GeV}$.

We will argue below that all of these issues can be (are) overcome at RHIC-II.

\section{THEORETICAL CONSIDERATIONS AND PREDICTIONS}

\subsection{Objectives and Framework}

Ample evidence for (early) thermalization of the matter formed in (semi/central) $\sqrt{s_{N N}}=200 \mathrm{GeV} \mathrm{Au}+\mathrm{Au}$ collisions from current RHIC data provides the necessary prerequisite to validate the study of the QCD phase diagram. So far, most of the deduced features pertain to bulk matter properties, including large energy densities, $\epsilon$, well above the critical density extracted from lattice QCD [24]. 
In the following subsections, we will sketch a theoretical framework that provides the basis for gaining decisive new insights in three areas: temperature measurements; vector meson spectral functions; and the origin of mass and chiral symmetry restoration.

Explicit temperature extraction has so far been restricted to hadro-chemical and -thermal freezeout, see Fig. 4. The goal is to establish early temperatures well above $T_{c}$, feasible with photons and dileptons at a typical energy/momentum/invariant-mass scale of $1<M<3 \mathrm{GeV}$. Together with information on the energy and/or entropy density, the effective number of degrees of freedom, $d_{\text {of }}$, may be accessed since $\epsilon=\frac{\pi^{2}}{30} d_{\text {of }} T^{4}$ and $s=\frac{2 \pi^{2}}{45} d_{\text {of }} T^{3}[26,27,28]$.

Dilepton invariant-mass spectra are invaluable and unique means of extracting microscopic information on the constituents of the medium. Modifications of low-mass vector mesons $(V=\rho, \omega$, and $\phi)$ in hot and/or dense hadronic matter have been extensively studied theoretically (see Refs. [29,30,31,32,33] for reviews), largely triggered by the intriguing excess radiation observed at the CERN-SPS $[7,10]$. At RHIC, for the first time, these measurements will be performed in an environment that is close to net baryon-free, which will provide important tests of the relevant mechanisms underlying the predicted medium effects. In addition, the higher initial temperatures achieved at RHIC will more directly access radiation from the (s)QGP and thus be more sensitive to suggested resonance/bound-state formation above $T_{c}$ in the vector channel [34,35,36,37] (see Fig. 5), relative to more indirect probes of resonances, e.g. quantitative analyses of energy loss [38] and hadron elliptic flow, especially in the charm sector [39]), or systematics of charmonium regeneration [40].

A more ambitious goal is to infer signatures of the phase transition, requiring the study of order parameters. For the chiral transition, these are e.g. quark condensates, see Fig. 6, the constituent quark mass and the pion decay constant. While none of these are (viable) observables, the condensate structure of the in-medium ground state is encoded in its hadronic excitations. It is thus necessary to establish connections between the in-medium vector correlator measured in dilepton spectra and order parameters.

All of the above are inevitable consequences of QGP formation, albeit mostly nonperturbative in nature. While data interpretation will require the application of phenomenological approaches, well-defined links to finite-temperature lattice QCD computations and symmetry constraints are essential for the deduction of meaningful results.

We emphasize that thermal production rates for photon and dilepton spectra can be cast into a uniform theoretical framework according to 


$$
\begin{aligned}
& q_{0} \frac{d N_{\gamma}}{d^{4} x d^{3} q}=-\frac{\alpha}{\pi^{2}} f^{B}\left(q_{0} ; T\right) \operatorname{Im}_{\mathrm{em}}^{T}\left(q_{0}=q ; \mu_{B}, T\right) \\
& \frac{d N_{e^{+} e^{-}}}{d^{4} x d^{4} q}=-\frac{\alpha^{2}}{M^{2} \pi^{3}} f^{B}\left(q_{0} ; T\right) \operatorname{Im} \Pi_{\mathrm{em}}\left(M, q ; \mu_{B}, T\right)
\end{aligned}
$$

where the key quantity is the retarded electromagnetic correlation function, $\Pi_{\mathrm{em}}[42,43]$. In the vacuum, this function can be measured in $e^{+} e^{-}$annihilation and decomposes into two regimes: at masses above $M=\sqrt{q^{2}} \simeq 1.5 \mathrm{GeV}$, the strength of the EM spectral function, $\operatorname{Im} \Pi_{\mathrm{em}}^{\mathrm{vac}}$, is rather accurately determined by perturbation theory, i.e. annihilation into $q \bar{q}$ pairs with little impact from subsequent hadronization. At low mass the cross section is saturated by the light vector mesons $\rho, \omega$ and $\phi$ (vector dominance), i.e. nonperturbative resonance formation:

$$
\operatorname{Im} \Pi_{\mathrm{em}}^{\mathrm{vac}}(M)= \begin{cases}\sum_{V=\rho, \omega, \phi}\left(\frac{m_{V}^{2}}{g_{V}}\right)^{2} \operatorname{Im} D_{V}(M) & M<1.5 \mathrm{GeV}, \\ -\frac{M^{2}}{12 \pi}\left(1+\frac{\alpha_{s}(M)}{\pi}+\ldots\right) N_{c} \sum_{q=u, d, s} e_{q}^{2} & M \geq 1.5 \mathrm{GeV} .\end{cases}
$$

For thermal dilepton emission this implies that, on one hand, the low-mass region carries information on dynamical medium effects with a relative strength of the vector mesons approximately given by 11:1:2 for $\rho: \omega: \phi$, reflecting the vector-dominance couplings, $m_{V}^{4} / g_{V}^{2}$, or, equivalently, the dilepton decay widths. On the other hand, at intermediate mass a reasonably controlled emission strength provides the basis for probing the temperature. The temperature and volume dependence in space-time integrated dilepton spectra combine such that the prevalent low-mass contribution originates from temperatures around and below $T_{c}[44]$. At higher masses and energies, the exponential sensitivity of the Bose factor strongly biases contributions toward high temperatures. Similar considerations apply to thermal photon transversemomentum spectra, corroborating the feasibility of realizing the three basic objectives listed above. Note that the leading-order contribution to the dilepton rate at sufficiently large invariant mass, $M>1.5 \mathrm{GeV}$, is $\mathcal{O}\left(\alpha_{s}^{0}\right)$ while a nonzero photon rate requires processes of at least $\mathcal{O}\left(\alpha_{s}\right)$.

In rough accord with our physics objectives, we adopt the following classification of regimes in dilepton invariant mass, $M_{l l}$, or photon transverse momentum, $q_{t}$ :

- the low-mass region (LMR), $M_{l l}<1.1 \mathrm{GeV}$ (vector meson decays);

- the intermediate-mass region (IMR), $1.1<M_{l l}<3 \mathrm{GeV}$ (continuum radiation, QGP emission, resonances?);

- the high-mass region (HMR), $M_{l l}>3 \mathrm{GeV}$ (primordial emission and heavy quarkonia). 


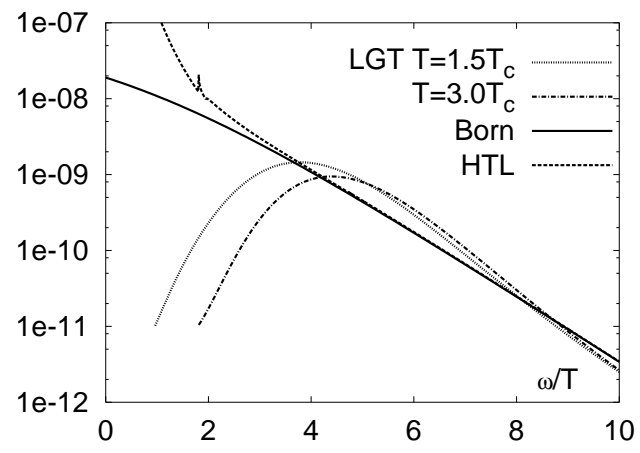

Fig. 7. Thermal dilepton production rates in the QGP as evaluated in quenched lattice gauge theory at zero three-momentum (dotted and dash-dotted line) [45]. The solid and dashed lines are pQCD calculations of the rate in the Born approximation, $q \bar{q}$ annihilation to leading order, $\mathcal{O}\left(\alpha_{s}^{0}\right)$, and with hard-thermal-loop corrections [46] respectively.

\subsection{QCD Lattice Results}

Thermal dilepton rates have been studied in lattice QCD within the quenched approximation [45]. The computation of the finite-temperature Euclidean correlators in the vector channel is supplemented by a transformation into the time-like regime using the maximum entropy method, after which the dilepton rate follows from Eq. (2). The results for zero three-momentum at two different temperatures above $T_{c}$ are compared to perturbative calculations [46] in Fig. 7. The lattice rates are quite comparable to the perturbative ones at high energy, while the enhancement at $\omega \simeq 5 T$ could be related to resonance formation in the QGP. Toward small energies, the lQCD rates drop substantially and deviate markedly from the perturbative calculations, which, in the HTL approximation, even diverge. Since the HTL calculation is of nontrivial order in $\alpha_{s}$, the HTL divergence is closely related to a non-vanishing thermal photon rate. Recent lattice computations of the vector correlator at finite 3momentum $[47,48]$ confirm that the rates in Fig. 7 lead to vanishing photon emission. If this seemingly unrealistic feature is induced by lattice artifacts, the decrease of the dilepton rate toward small $\omega$ may not hold, leading to better agreement with HTL extrapolations, consequently affecting the interpretation of the "resonance" structure. The ultra-soft limit of the EM correlator can be further studied by its relation to the electric conductivity, $\sigma_{\mathrm{em}}$, via the Kubo formula,

$$
\sigma_{\mathrm{em}}(T)=\frac{e^{2}}{3} \frac{\partial}{\partial q_{0}} \operatorname{Im} \Pi_{\mathrm{em}}^{T}\left(q_{0}, q=0 ; \mu_{B}, T\right) .
$$

The correlators found in Ref. [45] correspond to a vanishing conductivity. A different method employed in Ref. [49] leads to a finite, large value of $\sigma_{\mathrm{em}}$, $\sigma_{\mathrm{em}}(T) \simeq 7 \sum e_{q}^{2} T$, see Ref. [48]. It would be very interesting to compare this 
result to the conductivity underlying the soft photon emission rates used in the description of the low-momentum WA98 data [18], see the lower panel of Fig. 3. Along these lines, $\sigma_{\mathrm{em}}$ has been evaluated in a low-temperature pion gas within chiral perturbation theory [50].

Another quantity of interest is the susceptibility associated with conserved quantum numbers, i.e., derivatives of the thermodynamic free energy with respect to a chemical potential,

$$
\chi_{X}=-\frac{\partial^{2} \Omega}{\partial \mu_{X}^{2}} .
$$

These susceptibilities have been evaluated in lattice QCD for quark and isospin chemical potentials [51]. When extrapolated into the finite- $\mu_{q}$ plane, the isoscalar quark susceptibility develops a maximum while the isopin susceptibility remains monotonic. Since the susceptibilities can be related to the space-like static limit of the corresponding correlation function in the $\omega$ and $\rho$ channels, valuable information on the soft part of the spectral functions may be inferred [52] or at least tested for a given model. Whether the lattice results for the isoscalar susceptibility are related to an in-medium reduced $\omega$ mass e.g. via a $\sigma-\omega$ mixing mechanism close to the QCD critical endpoint, remains to be seen. Interestingly, recent experiments on nuclear $\omega$ photoproduction have provided evidence of $\omega$ mass reduction in nuclear matter [53]. A similar line of reasoning for the $\rho$ suggests that its mass is not much affected approaching the critical point [54].

\subsection{Sum Rules}

Besides direct lQCD calculations of dilepton and photon rates, unanticipated in the immediate future, model-independent information and effective-model constraints are encoded in (energy-weighted) sum rules relating (integrated moments of) spectral functions to vacuum expectation values of composite quark and gluon operators, "condensates", or (partial) conservation laws of (axial-) vector currents. These sum rules are thus prime examples of connecting hadronic excitations to the underlying ground-state structure (symmetrybreaking pattern), including order parameters. We will briefly discuss two classes of sum rules. 


\subsubsection{QCD Sum Rules}

QCD sum rules (QCDSRs) [55] are based on the analyticity of correlation functions, resulting in the dispersion relation

$$
\int d \omega \frac{\operatorname{Im} \Pi_{\mathrm{em}}(\omega)}{\omega-q_{0}}=\sum_{n} \frac{C_{n}}{Q^{2 n}} .
$$

The left-hand-side involves an integral over a (hadronic) spectral function in the time-like regime plus possible subtractions not indicated here. The righthand-side is an expansion in space-like momenta, $1 / Q^{2}\left(Q^{2} \equiv-q^{2}>0\right)$, with leading perturbative terms and nonperturbative effects encoded in Wilson coefficients, $C_{n}$, via quark and gluon condensates of dimension increasing with $n$. When applied to the light vector mesons, $\rho$ and $\omega$, at finite temperature and/or density [56], it turns out that the largest sensitivity resides in the medium dependence of the four-quark condensates, $\left\langle(\bar{q} q)^{2}\right\rangle$. Unfortunately, rather little is so far known about their temperature dependence from lattice QCD. The usual assumption is to factorize the four-quark condensates into a product of two-quark condensates corresponding to the assumption of "ground-state dominance" with an extra parameter, $\kappa$, usually fixed in the vacuum, representing correlation effects. An application of QCDSRs to the $\rho$ meson in cold nuclear matter is shown in Fig. 8 [58], indicating that the finite-density decrease of the condensates mandates a "softening" of the $\rho$ spectral function. The required low-mass enhancement can be satisfied by an increasing width, a decreasing mass, or a suitable combination thereof as indicated by the "allowed regions" enclosed by the bands in the right panel of Fig. 8, see Refs. $[57,59,60]$.

\subsubsection{Chiral Sum Rules}

Chiral sum rules (CSRs) $[61,62]$ have been derived prior to QCD from current algebra and chiral Ward identities. The (partial) conservation of the (axial-) vector-isovector currents leads to relations between the pion decay constant, $f_{\pi}=92 \mathrm{MeV}$ (an order parameter of chiral symmetry breaking), to moments of differences between pertinent spectral functions. In vacuum they are 4

$$
\int_{0}^{\infty} \frac{d s}{s^{2}}\left[\rho_{V}^{\mathrm{vac}}(s)-\rho_{A}^{\mathrm{vac}}(s)\right]=f_{\pi}^{2} \frac{\left\langle r_{\pi}^{2}\right\rangle}{3}-F_{A}
$$

4 The form of the sum rules in Eqs. (7)-(10) applies to the chiral limit of vanishing current light-quark masses and pion mass. Corrections to the second Weinberg Sum Rule (WSR), Eq. (9), may not be small $[63,64,65]$. The first WSR, Eq. (8), does not seem to be affected by finite quark mass corrections. 

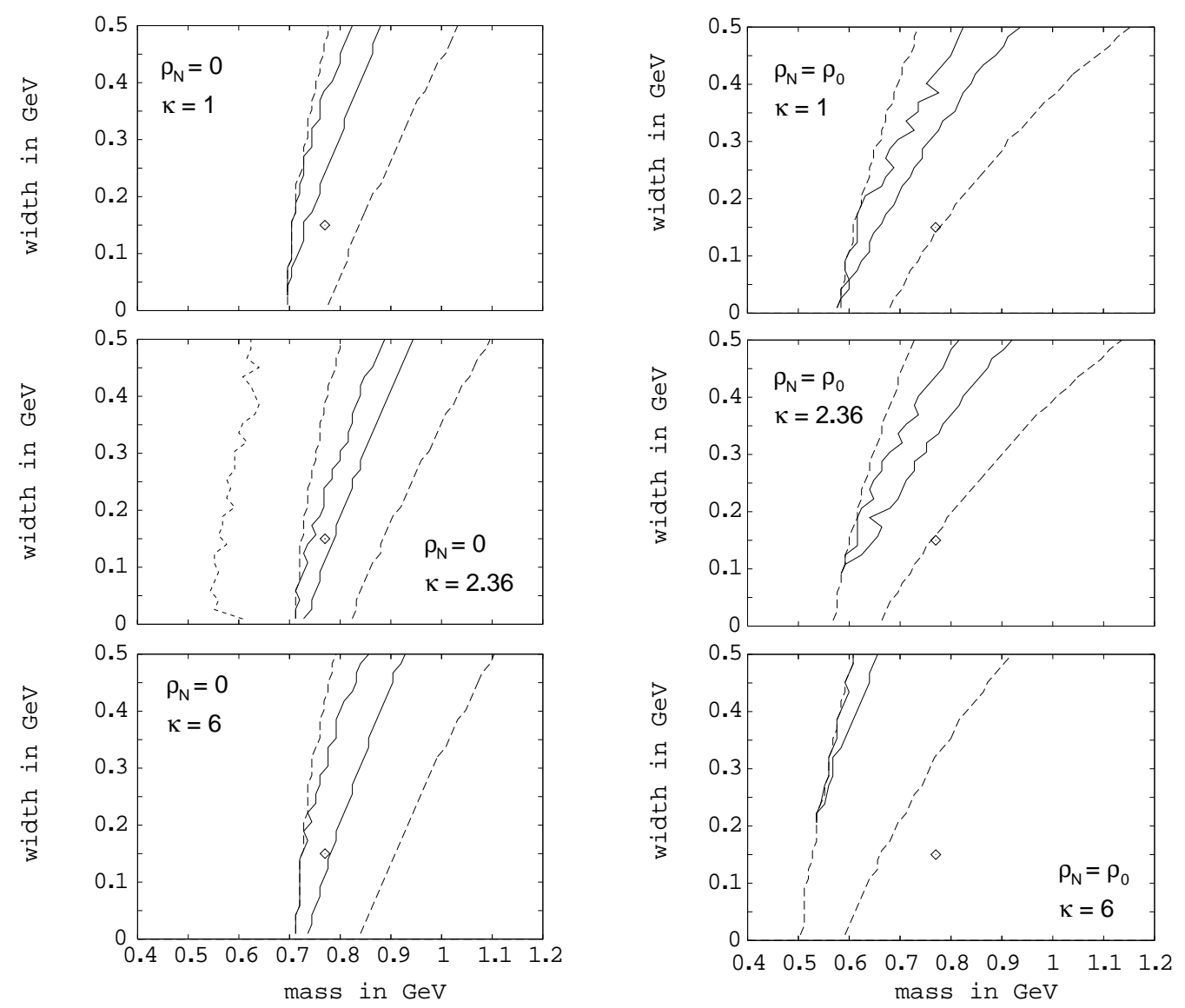

Fig. 8. QCD sum rule constraints on Breit-Wigner $\rho$ spectral functions in the mass-width plane [58]. The bands indicate regions of mass and width values for which the deviation between the left-hand and right-hand sides of Eq. (6) is below $0.2 \%$ (within the solid lines) or below $1 \%$ (within the dashed lines). The left column is for the vacuum case, which is used to determine the "correlation parameter", $\kappa$ in the four-quark condensate while the right column is at nuclear-matter density (diamond: mass and width of the free $\rho$ meson).

$$
\begin{aligned}
& \int_{0}^{\infty} \frac{d s}{s}\left[\rho_{V}^{\mathrm{vac}}(s)-\rho_{A}^{\mathrm{vac}}(s)\right]=f_{\pi}^{2}, \\
& \int_{0}^{\infty} d s\left[\rho_{V}^{\mathrm{vac}}(s)-\rho_{A}^{\mathrm{vac}}(s)\right]=0,, \\
& \int_{0}^{\infty} s d s\left[\rho_{V}^{\mathrm{vac}}(s)-\rho_{A}^{\mathrm{vac}}(s)\right]=-2 \pi \alpha_{s}\langle\mathcal{O}\rangle,
\end{aligned}
$$

where $\left\langle r_{\pi}^{2}\right\rangle$ is the pion charge radius squared, $F_{A}$ the axial vector form factor in the radiative pion decay, $\pi \rightarrow l \bar{\nu}_{l} \gamma$. The vacuum vector and axial vector spectral functions are

$$
\rho_{V, A}^{L, T}=-\frac{1}{\pi} \operatorname{Im} \Pi_{V, A}^{L, T}
$$


In Eq. (10), obtained in Ref. [66], $\langle\mathcal{O}\rangle$ denotes a four-quark condensate. In the factorization approximation, $\langle\mathcal{O}\rangle=(16 / 9)\langle\bar{q} q\rangle^{2}$. The direct connection of the CSRs to the vector correlator renders them particularly valuable in the context of dilepton production. The assessment of in-medium effects requires their extension to finite temperature, elaborated in Ref. [66]. Due to loss of Lorentz invariance when specifying the thermodynamic rest frame, the original vacuum results become energy sum rules at fixed three-momentum and split into longitudinal $(L)$ and transverse $(T)$ components of the correlators and quark condensate:

$$
\begin{aligned}
\int_{0}^{\infty} \frac{d q_{0}^{2}}{\left(q_{0}^{2}-q^{2}\right)} \Delta \rho^{L}\left(q_{0}, q\right) & =0, \\
\int_{0}^{\infty} d q_{0}^{2} \Delta \rho^{L, T}\left(q_{0}, q\right) & =0, \\
\int_{0}^{\infty} q_{0}^{2} d q_{0}^{2}\left[\Delta \rho^{L}\left(q_{0}, q\right)+2 \Delta \rho^{T}\left(q_{0}, q\right)\right] & =-4 \pi \alpha_{s}\left[\left\langle\left\langle\mathcal{O}_{\mu}^{\mu}\right\rangle\right\rangle+2\left\langle\left\langle\mathcal{O}^{00}\right\rangle\right\rangle\right],
\end{aligned}
$$

where $\Delta \rho \equiv \rho_{V}-\rho_{A}$ and $\langle\langle\cdot\rangle\rangle$ denotes an in-medium expectation value. The transverse and longitudinal components of the spectral functions are given in terms of standard projection operators,

$$
\rho_{V, A}^{\mu \nu}=\rho_{V, A}^{T} P_{T}^{\mu \nu}+\rho_{V, A}^{L} P_{L}^{\mu \nu}
$$

where the pionic piece, $\rho_{\pi}^{\mu \nu}=f_{\pi}^{2} q^{2} \delta\left(q^{2}\right) P_{L}^{\mu \nu}$ in vacuum, has been included in the longitudinal axial vector channel. In medium, the pion spectral function is subject to medium modifications as well. The in-medium Weinberg-type sumrules (12)-(14) impose stringent constraints on both temperature and energymomentum dependencies through the moments of chiral hadronic models for vector and axial vector spectral functions. The model-independent connection to lattice QCD can be implemented by employing the pertinent temperature dependencies of the pion decay constant and four-quark condensates which, in principle, are easier to compute than full spectral functions.

We now turn to a more concrete discussion with examples of how to realize the three main points outlined at the beginning of this Section. 

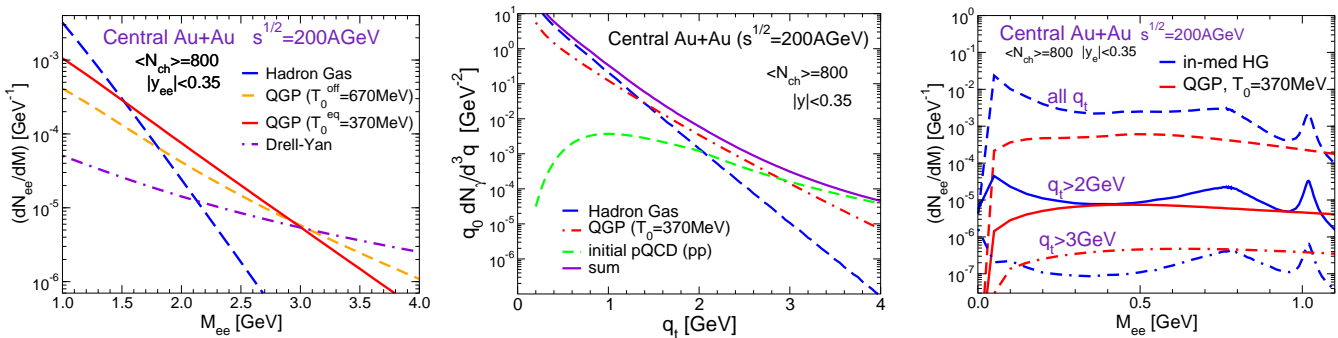

Fig. 9. Comparison of electromagnetic emission spectra from QGP and HG at intermediate $M_{l l}$ and $q_{t}$ in central $\mathrm{Au}+\mathrm{Au}$ collisions at $\sqrt{s_{N N}}=200 \mathrm{GeV}$. Left panel: IMR dileptons [67] including primordial Drell-Yan annihilation. Middle panel: direct photons [19] including primordial pQCD contributions. Right panel: low-mass dileptons with pair $q_{t}$ cuts. Note that the IMR dilepton spectrum (left) neither includes contributions from the $\phi$ nor a cut on the single-electron rapidity. The single-electron rapidity cut has been applied to the low mass region, causing a significant reduction in the yield. On the other hand, the low mass spectra (right) does not include dilepton emission from four-pion and higher states that dominate hadronic emission beyond the $\phi$ mass.

\subsection{Temperature and Degrees of Freedom}

\subsubsection{Suitable Kinematic Regimes}

Thermal emission rates for electromagnetic radiation, Eqs. (1) and (2), can in principle be used to "infer" the temperature of thermalized matter in heavyion collisions if: (i) the emission strength represented by the EM correlator is reasonably well determined so that the $T$ dependence essentially resides in the Bose factor and (ii) a kinematic window can be identified where radiation from a reasonably well-defined temperature regime prevails. Ideally, these conditions are met at the highest masses and energies where the correlators can be reliably evaluated in pQCD and thermal emission from the earliest phases dominates. In practice, however, the high-mass/energy region is dominated by dileptons and photons from primordial hard $N N$ collisions. At lower masses, the lower temperature contributions increase substantially. A more comprehensive discussion of the various sources will be given below.

Fig. 9 shows an example of predictions for space-time integrated photon and dilepton spectra at intermediate masses and transverse momenta in central $\sqrt{s_{N N}}=200 \mathrm{GeV} \mathrm{Au}+\mathrm{Au}$ collisions $[19,67]$. The thermal spectra are decomposed into QGP and hadronic (HG) emission from an isentropically expanding thermal fireball assuming a critical temperature of $T_{c}=180 \mathrm{MeV}$ [68] where the total entropy is fixed to reproduce the observed hadron multiplicities at chemical freeze-out, $\left.\mu_{N}^{\text {ch }}, T_{\mathrm{ch}}\right)=(25,180) \mathrm{MeV}$. The pQCD photon rates, to leading order in $\alpha_{s}$ [69], and HTL-resummed dilepton rates [46] are convoluted over a chemically-equilibrated QGP assuming a formation time of $\tau_{0}=1 / 3 \mathrm{fm} / c$, translating into $\bar{T}_{0}=370 \mathrm{MeV}(670 \mathrm{MeV}$ if the initial parton 
densities are assumed to be undersaturated). Uncertainties in the longitudinal expansion, affecting the QGP lifetime, can induce changes of the QGP spectra by up to $30 \%$. The sensitivity to $\tau_{0}$ is larger, especially at masses above $2 \mathrm{GeV}$ [67]. The hadronic emission spectra include in-medium modifications of the EM correlator, see Sec. 2.5 for details, as well as chemical off-equilibrium in the hadronic evolution until thermal freeze-out, see Sec. 2.6. Three regimes emerge where QGP radiation outshines both HG and primordial emission, see Fig. 9):

(i) three-momentum integrated dilepton spectra at intermediate mass, $1.5 \leq$ $M \leq 3 \mathrm{GeV}$ (left panel);

(ii) direct photon spectra at intermediate transverse momentum, $1.5 \leq q_{t} \leq$ $3 \mathrm{GeV}$ (middle panel);

(iii) low-mass dilepton spectra at $q_{t} \geq 2 \mathrm{GeV}$ ("low-virtuality" photons; right panel).

In practice a careful assessment of additional sources, including "pre-equilibrium" contributions and jet-plasma interactions such as Bremsstrahlung off quark jets or Compton scattering of gluon jets [70,71], is mandatory before firm conclusions about the thermal component can be reached. We note that the predictions for the thermal photon spectra, together with jet-plasma interactions and primordial photons extracted from $p p$ collisions, compare favorably with preliminary RHIC direct photon data [72], as discussed later. Recent calculations of the jet-plasma component suggest that it exceeds thermal emission for real and virtual photon transverse momenta $q_{t} \geq 3-4 \mathrm{GeV}[70,71]$. See Section 3.5 for a possible strategy for disentangling the different components.

\subsubsection{Direct Photons and Current RHIC Data}

To illustrate the uncertainties in and the required precision of a "temperature measurement", we describe an analysis of recent direct photon spectra in central $200 \mathrm{GeV} \mathrm{Au+Au} \mathrm{collisions} \mathrm{at} \mathrm{RHIC.} \mathrm{The} \mathrm{left} \mathrm{panel} \mathrm{of} \mathrm{Fig.} 10$ compiles several model calculations of thermal photon production mostly based on hydrodynamic evolution. The maximum initial temperature, $T_{0}$, is quoted: Srivastava et al. [73], $\tau_{0} \approx 0.2 \mathrm{fm} / c$ and $450<T_{0}<660 \mathrm{MeV}$; Alam et al. [74], $\tau_{0}=0.5 \mathrm{fm} / c, T_{0}=300 \mathrm{MeV} 5$, Räsänen et al. [76], $\tau_{0}=0.17 \mathrm{fm} / c$ and $T_{0}=580 \mathrm{MeV}$; Turbide et al. [19], expanding fireball with $\tau_{0}=0.33 \mathrm{fm} / \mathrm{c}$ and $\left\langle T_{0}\right\rangle=370 \mathrm{MeV}$; d'Enterria and Peressounko [28], $\tau_{0}=0.15 \mathrm{fm} / c$ and $T_{0}=590 \mathrm{MeV}$; see also Steffen and Thoma [77], $\tau_{0}=0.5 \mathrm{fm} / c\left\langle T_{0}\right\rangle=300$ $\mathrm{MeV}$. For similar initial conditions, the total thermal yields in these calculations are compatible both with the data and each other within a factor of

5 Alam et al. have recently [75] recomputed their hydrodynamic yields using higher initial temperatures, $T_{0}=400 \mathrm{MeV}$ at $\tau_{0}=0.2 \mathrm{fm} / c$, to improve the agreement with the data. 

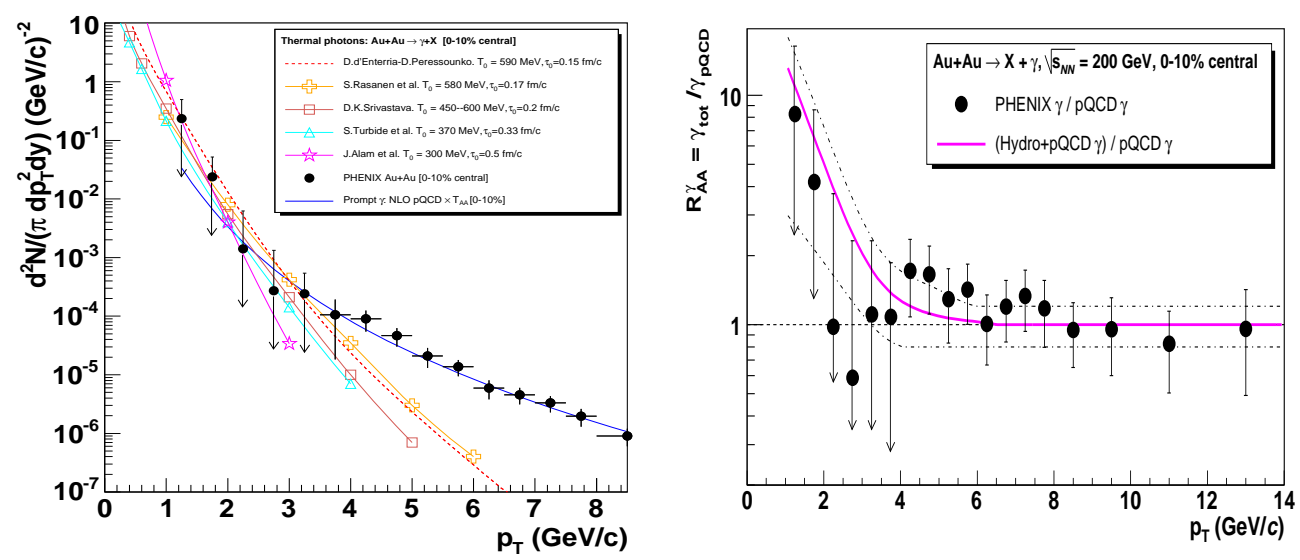

Fig. 10. Left panel: Thermal photon spectra for central $\mathrm{Au}+\mathrm{Au}$ reactions at $\sqrt{s_{N N}}=200 \mathrm{GeV}$, computed within different models (see text), compared to the expected pQCD prompt $\gamma$ yields ( $T_{A A}$-scaled NLO $p p$ calculations [9]: solid line, no symbols) and to the experimental total direct photon spectrum measured by PHENIX [8]. Right panel: Direct photon "nuclear modification factor", Eq. (16), for the $0-10 \%$ most central $\mathrm{Au}+\mathrm{Au}$ reactions at $\sqrt{s_{N N}}=200 \mathrm{GeV}$. The solid line is the ratio resulting from a hydro+pQCD model [28]. The points show the PHENIX data [8] over the same NLO yields while the dashed-dotted curves indicate the theoretical uncertainty on the NLO calculations.

$\sim 2$. While this confirms the dominant role of thermal radiation in the window $p_{T} \simeq 1.5-3 \mathrm{GeV}$, it shows that more quantitative, in-depth comparisons are required to disentangle the underlying assumptions on evolution model (boost-invariant hydrodynamics with or without transverse expansion, thermal fireballs, etc.) from production rates to narrow down the viable $T_{0}$ range. The excess over that expected from primordial $N N$ collisions, the "prompt" contribution, is better illustrated by the nuclear modification factor, $R_{A A}^{\gamma}$, the ratio of the direct photon spectra in $A A$ collisions relative to either the pQCD prediction for $p p$ collisions, scaled by the nuclear overlap interval, $T_{A A}$, or, preferably, to $p p$ spectra measured in the same experiment,

$$
R_{A A}^{\gamma}\left(p_{T}\right)=\frac{d N_{A u A u}^{\mathrm{tot} \gamma} / d p_{T}}{T_{A A} \cdot d \sigma_{p p}^{\mathrm{pQCD} \gamma} / d p_{T}},
$$

shown on the right side of Fig. 10. The data are consistent with a significant excess over the next-to-leading order (NLO) pQCD predictions. However, we emphasize that below $p_{T} \approx 4 \mathrm{GeV}$ it is not yet clear to what extent the NLO predictions in the denominator of Eq. (16) are applicable 6 . In this regime, the calculated prompt yields are dominated by jet Bremsstrahlung, determined from the parametrized parton-to-photon GRV form factor [78], rather poorly known at the pertinent $p_{T}$. The standard scale uncertainties in the NLO pQCD

6 The denominator may be modified by the "isospin effect" or other factors, see Sec. 3.4. 
calculations are $\pm 20 \%$ for $p_{T} \geq 4 \mathrm{GeV}$ but could become as large as ${ }_{-200}^{+50} \%$ for $1 \leq p_{T} \leq 4 \mathrm{GeV}$, as indicated by the dash-dotted lines on the right side of Fig. 10. Obviously, precise measurements of the direct photon baseline spectra in $p p$ and $\mathrm{d}+\mathrm{Au}$ collisions at $\sqrt{s}=200 \mathrm{GeV}$ above $p_{T}=1 \mathrm{GeV}$ are essential for quantifying a thermal signal in $\mathrm{Au}+\mathrm{Au}$ collisions.

The measured slope of the resulting thermal photon spectrum, $T_{\text {eff }}$, does not directly reflect the temperature of the hot matter since photons are emitted throughout the space-time volume of the evolving matter, implying varying temperatures as well as blue shifts due to collective expansion. Nevertheless, a correlation between the apparent photon slope and the maximum temperature attained in the system persists, as in the recent hydrodynamical study within [28]. The measured $T_{\text {eff }}$ provides an empirical link to the effective number of degrees of freedom of the system via $d_{\mathrm{of}}=\left(30 / \pi^{2}\right)\left(\epsilon / T_{\text {eff }}^{4}\right)$ or $d_{\text {of }}=\left(45 / 2 \pi^{2}\right)\left(s / T_{\text {eff }}^{3}\right)[28]$. The initial maximum energy and entropy densities are difficult to access experimentally. Indeed, all observables related to the initial energy and entropy densities such as the total transverse energy, the total particle multiplicity, and the colored-particle density encountered by quenched jets on their path through the medium are related to space-time averaged quantities. Information on the temperature dependence of $d_{\text {of }}$ can, however, be obtained via centrality and $\sqrt{s_{N N}}$ dependencies. It is possible to discriminate a QGP-like equation of state with a fixed $d_{\text {of }}$ above $T_{c}$ from a hadronic resonance gas with a rapidly rising number of degrees of freedom, by establishing the dependence of $T_{\text {eff }}$ on the charged particle pseudorapidity density [28]. As a further consistency check, one can relate $d_{\text {of }}$ to suitable powers of the energy and entropy densities, $d_{\text {of }} \propto s^{4} / \epsilon^{3}[27]$.

\subsubsection{Chemical Off-Equilibrium}

While rapid thermalization of the matter at full RHIC energy is fairly well established, its composition in terms of quark and gluon degrees of freedom (chemical equilibration) is much less clear, as are the equilibration mechanisms themselves 7 . From the prevalence of gluons in the relevant $x$-range of the incoming nuclei at midrapidity, one might expect the early matter to be a gluon plasma (GP), as is routinely assumed e.g. in jet-quenching by radiative energy loss [79]. However, recent calculations of $q \bar{q}$ pair production within the classical fields generated by the incoming Au nuclei indicate a rather fast approach to chemical equilibrium [80]. This rapid equilibriation could have important consequences for disentangling the relevant fast parton energy loss mechanism (with a significantly reduced radiative loss for quarks due to the smaller color charge). EM probes are an obvious means of testing chemical

7 The expansion in hydrodynamic simulations is mostly driven by the ratio of pressure to energy density, $P / \epsilon$, where $d_{\text {of }}$ essentially drops out. 
equilibration since gluons carry no electric charge $[67,81,82,83,84]$. LO pQCD processes $\left(q+g \rightarrow \gamma+X, q \bar{q} \rightarrow l^{+} l^{-}\right)$in a thermally equilibrated but chemically off-equilibrium QGP suggest that the photon and dilepton production rates scale with $\lambda_{g} \lambda_{q, \bar{q}}$ and $\lambda_{q} \lambda_{\bar{q}}$, respectively, where $\lambda_{i}$ are fugacities characterizing the deviation of the parton densities from chemical equilibrium $\left(\lambda_{q}=\lambda_{\bar{q}}=\lambda_{g}=1\right)$. At RHIC, a typical GP initial state with subsequent evolution using inelastic pQCD reaction rates starts from $\lambda_{g} \simeq 1 / 3, \lambda_{q, \bar{q}}<0.1$ evolving to values of $\lambda \simeq 0.5$ or larger $[84,85,86]$. However, in an isentropic expansion with fixed initial entropy, undersaturated matter implies significantly higher initial temperatures at otherwise identical conditions; e.g. for central $\mathrm{Au}+\mathrm{Au}$ collisions at RHIC, with $\tau_{0}=1 / 3 \mathrm{fm} / c, T_{0} \simeq 370 \mathrm{MeV}$ in a chemically-equilibrated system relative to $T_{0} \simeq 670 \mathrm{MeV}$ in a system off chemical equilibrium. In the photon spectra, reduced fugacities in the emission rate are largely compensated by the higher temperatures in the QGP evolution with only a slight hardening of the slope parameter [26]. This effect appears to be more pronounced for thermal dilepton spectra, see the left-hand side of Fig. 9. Proving that a harder slope is evidence for a GP at RHIC is further complicated by the fact that the thermal yields are still fairly sensitive to the assumed thermalization time, $\tau_{0}$. A decrease in $\tau_{0}$ with chemical equilibration not only decreases the slope of the thermal spectrum but also increases the yield. Identifying a GP or more generally, the number of degrees of freedom above $T_{c}$ with thermal dileptons will thus necessarily involve a quantitative assessment of both slope and absolute magnitude of the thermal spectrum, after "removal" of non-thermal sources including Drell-Yan dileptons, correlated open-charm decays, as well as pre-equilibrium and jet-plasma interactions. In the following section we briefly summarize recent progress on the last two sources.

\subsubsection{Pre-Equilibrium and Jet-Plasma Emission}

Dilepton emission subsequent to the initial hard $N N$ collisions but before the assumed $\tau_{0}$, the so-called pre-equilibrium contribution, can be addressed in a parton cascade approach. A corresponding calculation [89] predicted large emission rates, which, in fact, overestimate preliminary PHENIX data [90] (Fig. 11). However, if Landau-Pomeranchuk-Migdal (LPM) interference effects are included, the yield is appreciably suppressed [87], indicating that the combined initial pQCD plus pre-equilibrium yield is not very different from the pQCD contribution alone (Fig. 11), with the thermal yield dominating at $p_{T} \leq 2.5 \mathrm{GeV}$, similar to the center of Fig. 9. Also note that the calculated thermal spectra in Fig. 11 agree reasonably well with those in the center panel of Fig. 9. However, the present model dependencies will have to be further reduced to achieve enough sensitivity to discern the composition of the early matter and realize the desired temperature measurement. 


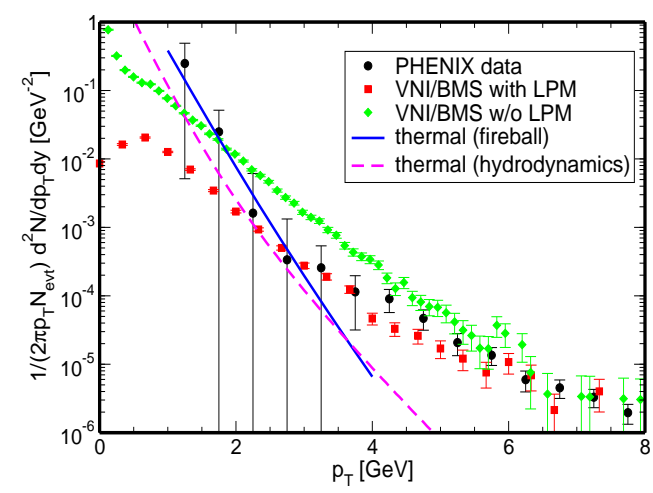

Fig. 11. Comparison of (early) photon emission from a parton cascade for central $200 \mathrm{GeV} \mathrm{Au+Au} \mathrm{collisions} \mathrm{[87]}$ with (squares) and without (diamond) LPM effects. Also shown are expanding fireball [88] and hydrodynamic [20] calculations of thermal photons, as well as preliminary data from PHENIX [90].

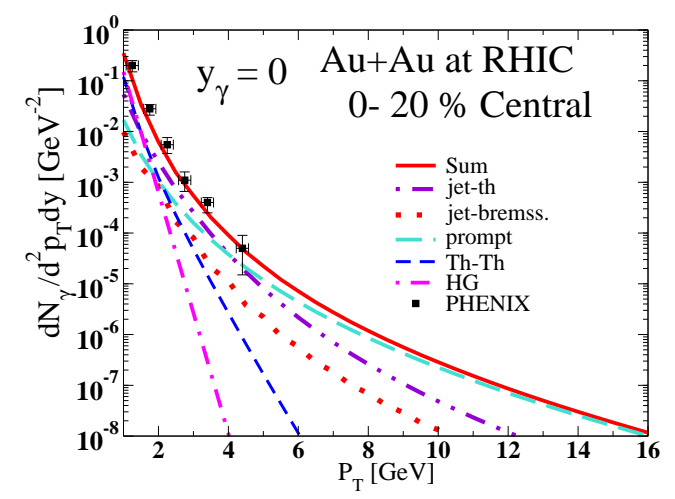

Fig. 12. Predictions for direct photon spectra combining pQCD prompt photons, jet-plasma interactions [70] and thermal radiation [19] compared to preliminary PHENIX data [72]. (Here "Th-Th" and "HG" are thermal radiation from the QGP and hadron gas respectively.)

Fig. 12 shows the combined prediction for direct photons from prompt (pQCD) production, jet-plasma interactions [70], and thermal QGP and HG radiation [19] (see center panel of Fig. 9) evaluated within the same expanding fireball. The comparison to preliminary low- $p_{T}$ PHENIX data [72] is quite encouraging. In this calculation, jet-plasma contributions exceed the thermal yield at $p_{T} \simeq 2 \mathrm{GeV}$, implying a rather narrow "QGP window". On the other hand, at $p_{T} \simeq 5 \mathrm{GeV}$, where jet-plasma radiation [70] is still significant, the experimental direct-photon $R_{A A}$ in the right panel of Fig. 10 does not indicate much room for enhancement over prompt pQCD photons. Note, however, that thermal and jet-induced radiation are not independent contributions since both are affected by the lifetime and temperature or number and energy density characterizing the QGP, imposing an additional consistency requirements. Furthermore, as emphasized in Refs. [70,71], the intensity of electromagnetic radiation from jet-plasma interactions is intimately related to radiative energy loss, i.e., jet quenching via gluon emission. In this context, the estimates for the jet-plasma photon and dilepton contribution in Refs. [70,71] constitute an upper limit, since high- $p_{T}$ pion suppression has been entirely attributed to radiative energy loss whereas recent calculations find a significant role played by elastic energy loss [91], even within the same formalism [92].

\subsection{In-Medium Spectral Functions below and above $T_{c}$}

In the mid 1990's, dilepton data from the CERN SPS triggered vigorous theoretical activity in trying to assess modifications of vector-meson properties in hot/dense (hadronic) matter. The focus has been on the $\rho$ meson due to 
its prevalent role in dilepton emission, see, e.g. Refs. [29,30,31,32,33] for recent reviews. In Secs. 2.5.1 and 2.5.2, we briefly summarize some of the main features and insights that have emerged over the last $\sim 10$ years.

\subsubsection{Hadronic Many-Body Theory and Chiral Virial Expansion}

Effective hadronic models for vector mesons should be compatible with basic symmetry principles, most notably electromagnetic gauge invariance, vectorcurrent conservation, and chiral symmetry 8 . In addition, it is essential that the underlying effective vertices are carefully constrained by phenomenological information such as hadronic and electromagnetic decay widths or scattering data. Measurements which provide information on in-medium effects near nuclear matter density are particularly valuable, e.g. photoabsorption data on both nucleons and nuclei $[93,94]$.

Most of the effective models with constraints built along the above lines have reached a reasonable degree of agreement with the data, generically predicting substantial broadening in matter with little mass shift 9 , characteristic of calculations both in cold nuclear matter $[59,95,96,97,98]$ and in hot and dense matter $[12,99]$, see Fig. 13 for two examples. Effective models also suggest that the effects of the baryonic component of the medium dominate over those from the mesonic one at comparable density, consistent with findings in large- $N_{c}$ QCD where meson-meson interactions are suppressed relative to meson-baryon ones).

The broadening of the spectral functions, amounting to a total width of $\sim 500 \mathrm{MeV}$ at nuclear matter saturation density and typically accompanied by a slight upward mass shift (left panel of Fig. 14), is in fair agreement with constraints from QCD sum rules [58], recall Fig. 8. When extrapolated to temperatures and densities close to the expected chiral transition, an almost complete "melting" of the $\rho$-resonance structure emerges. This is not only true for the net-baryon rich regime at SPS energies and below, but also in the central rapidity region at collider energies where the baryon chemical potential is small (center panel of Fig. 14). At the experimentally extracted chemical freezeout temperature, e.g. $T_{\mathrm{ch}} \simeq 180 \mathrm{MeV}$ at RHIC, an appreciable density of baryon-antibaryon, $B \bar{B}$, pairs is thermally excited [67] and mesons interact with both baryons and antibaryons. In addition, the notion of chemical freeze-

\footnotetext{
8 In many instances, little is known about the chiral structure of baryonic and mesonic resonance couplings, especially if no pions are involved.

9 A simple explanation of this feature is that imaginary parts of the in-medium self energies, which govern the broadening, are negative definite $(\operatorname{Im} \Sigma<0)$ and therefore strictly sum up whereas real parts, which induce mass shifts, change sign around a resonance. Real parts therefore tend to cancel if the system is characterized by a rich excitation spectrum, as is the case for a hadronic resonance gas.
} 


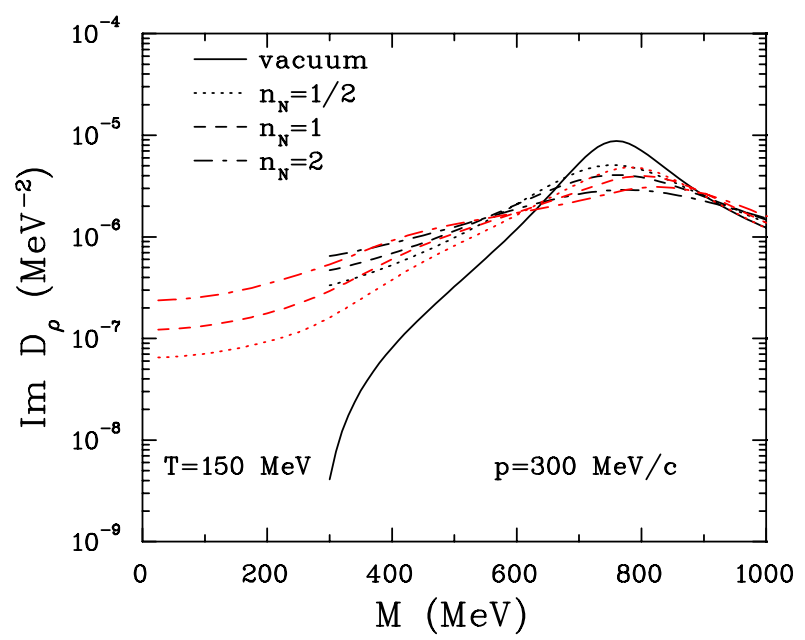

Fig. 13. Comparison of $\rho$ spectral functions in hot hadronic matter within a manybody calculation [12] (gray lines, extending to $M=0$ ) and an approach based on imaginary parts of $\rho$-hadron scattering amplitudes supplemented by dispersion relations to obtain the real parts [99] (black lines). Reasonable agreement between the in-medium results is observed, especially for nucleon densities, $n_{N} \leq 1$ (in units of the nucleon saturation density, $0.16 \mathrm{fm}^{-3}$ ).

out implies that baryon-antibaryon annihilation in the subsequent hadronic evolution is suppressed [101]. Thus, the relevant quantity for medium effects on vector mesons is the sum of the $B$ and $\bar{B}$ densities which, close to $T_{\mathrm{ch}}$, is quite comparable at $\mu_{B}=0$ and $\mu_{B}=250 \mathrm{MeV} 10$. The baryon-density effects on the $\rho$ are most pronounced at masses below $\sim 0.5 \mathrm{GeV}$ instead of at and above the free $\rho$ mass (compare the long-dashed and short-dashed lines in the center panel of Fig. 14). While the $\phi$ appears to be less sensitive to the baryonic component of the medium, this conclusion may be altered once a better understanding of recent photon- and proton-nucleus $\phi$ production data has been achieved [102,103].

The $\rho$ and possible $\omega$ "melting" has interesting implications that deserve further theoretical investigations:

(i) The very short mean-free path of the $\rho$ (and other hadrons) close to $T_{c}$ is suggestive of hadronic liquid formation [104]. Thus from a hadron structure objective, the matter properties in the phase transition region may change rather smoothly from a hadronic liquid to the sQGP liquid.

(ii) The hadronic in-medium EM correlator, based on a "melted" $\rho$, is surpris-

\footnotetext{
${ }^{10}$ Experimentally, the total baryon rapidity density, $d N_{B+\bar{B}}(y=0) / d y$, is indeed very comparable at the maximum SPS $\left(\sqrt{s_{N N}}=17.3 \mathrm{GeV}\right)$ and $\operatorname{RHIC}\left(\sqrt{s_{N N}}=\right.$ $200 \mathrm{GeV}$ ) energies. The total hadron rapidity density (mostly due to pions) is a factor of $\sim 2$ larger at RHIC, implying an accordingly lower total baryon density at the transition. However, most of the pertinent medium effects on the $\rho$ spectral function build up at densities at or below $\varrho_{0}$, see the left panel of Fig. 14.
} 

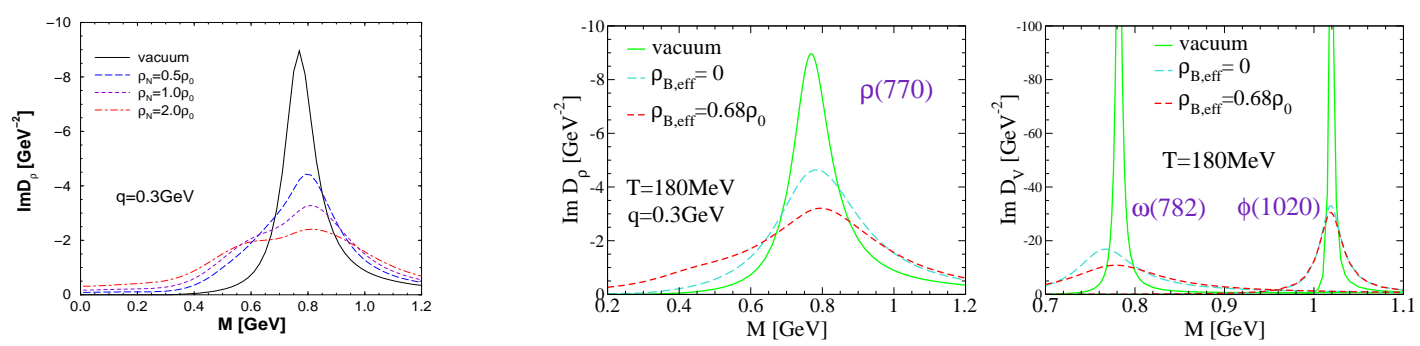

Fig. 14. In-medium vector meson spectral functions within hadronic many-body theory. Left: the $\rho$ in cold nuclear matter [100] at several densities proportional to the saturation density, $\varrho_{0}=0.16 \mathrm{fm}^{-3}$. Center: $\rho$ behavior at RHIC conditions close to $T_{c}$ with and without baryon effects [67]. Right: same as the center panel but for $\omega$ and $\phi[67]$.

ingly similar in shape and magnitude to the QGP correlator evaluated in HTL pQCD [46] at all masses [12,105]. This is also suggestive of a rather continuous transition from $\mathrm{HG}$ to QGP close to $T_{c}$, even on the level of spectral functions. The approximate coincidence of the bottom-up and topdown extrapolated hadronic and pQCD calculations, while not understood theoretically, enhances our confidence in applying these emission rates to fireball and hydrodynamic evolution across $T_{c}$, necessary for the space-time integrated thermal dilepton spectra, rendering the dilepton spectra rather insensitive to the exact value of $T_{c}$ [106].

Similar conclusions about the $\rho$ also emerge from the chiral virial approach [94] where medium effects on the vacuum vector correlator are evaluated within a pion- and nucleon-density expansion coupled with vacuum $V \pi$ and $V N$ scattering amplitudes constrained by chiral symmetry. The $\rho$ peak is quenched, though not broadened, and its low-mass shoulder is substantially enhanced, predominantly due to baryon effects in the heat bath [107]. This agreement, at least at low and moderate densities and temperatures, is a consequence of the constraints imposed on the underlying hadronic interactions.

\subsubsection{Dropping Mass}

Models involving dropping vector-meson masses [108] have recently been revisited within the so-called vector manifestation of chiral symmetry $[32,109,110]$. Using the Hidden Local Symmetry (HLS) framework, where the $\rho$ mass is generated via a Higgs mechanism, an alternative representation of the chiral group has been proposed in which the chiral partner of the pion is the longitudinal $\rho$ meson, the so-called "vector manifestation" of chiral symmetry instead of the conventional realization where the $\sigma$ is the chiral partner of the pion. The HLS approach results in a satisfactory vacuum phenomenology. A renormalization group analysis with hadronic loop effects reveals a fixed point with a vanishing vector coupling constant. When applied to the second 
order finite temperature chiral phase transition, matching the vector and axial vector correlators to the operator product expansion (space-like $q^{2}$ ) requires that bare $\rho$ mass vanish at the critical point, becoming degenerate with the pion mass, which persists when carried on-shell due to the fixed-point nature of the transition. The vector dominance model (VDM), which works well in the vacuum, is perhaps violated at finite temperature [110], suppressing $\pi \pi$ annihilation to dileptons via an intermediate $\rho$, replaced by direct annihilation via intermediate photons. The violation of vector dominance could make the observation of a dropping $\rho$ mass difficult in dilepton spectra, perhaps reconciling the new NA60 data [6,7] with a dropping-mass scenario. Ref. [111] calculates the dilepton rates in the vector manifestation scenario. Despite violation of the VDM, the rates clearly exhibit a $\rho$ peak with dropping mass, at least up to $T \sim 0.85 T_{c}$. We emphasize that the matching procedure is only valid sufficiently close to $T_{c}$ and that "ordinary" hadronic medium effects become dominant at lower temperatures. In the low-temperature limit, modification of the $\rho$ mass to leading order in temperature, $\mathcal{O}\left(T^{2}\right)$, is at variance with chiral symmetry. Thus, the notion of a "flash temperature" has been introduced [33,112], below which the "intrinsic" temperature dependencies of the parameters in the Lagrangian are void.

At the quark-antiquark level, interactions in the vector channel are believed to be rather weak since the $\rho$ mass is nearly twice the constituent quark mass. Finite temperature effects resulting in enhanced interactions and an accordingly reduced $\rho$ mass are not easily conceived [113]. (See Ref. [114] for an alternative view.) It would also be interesting to work out how the presence of hadronic many-body effects (especially baryons), as discussed in the previous section, affect the matching procedure and the resulting (axial) vector spectral functions.

\subsubsection{Resonances in the sQGP}

Another interesting development perhaps related to EM measurements are the conjectured hadronic bound states in the (s)QGP $[35,36]$. Detecting signatures of vector states above $T_{c}$ in the dilepton spectrum hinges on whether their mass is sufficiently large, $M_{V}\left(T \geq T_{c}\right)>1 \mathrm{GeV}$. As elaborated after Eq. (3), QGP radiation can only compete with or dominate contributions from the longer-lived and larger-volume hadronic phase at large masses, especially if the resonance structure depends on temperature. The lQCD spectral functions and dilepton rates shown in Figs. 5 and 7, respectively, indeed indicate resonances with masses in the $M \simeq 2 \mathrm{GeV} \simeq 10 T_{c}$ regime, roughly scaling with temperature. The existence of these states may be understood [35] to be due to heavy-quark quasiparticles bound by a rather strong color Coulomb-type attraction, also present in heavy quarkonium states. In this case, heavy-quark symmetry implies approximate degeneracy of vector ( $\rho$ " or $J / \psi$ ) and pseu- 


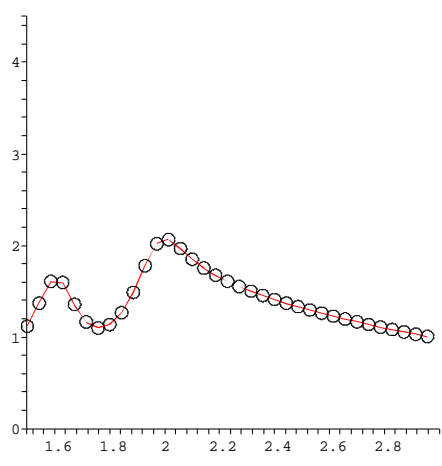

Fig. 15. Ratio of dilepton spectra at RHIC from a resonance-enhanced QGP rate to perturbative $q \bar{q}$ annihilation [37] obtained from the QGP and mixed phases of an expanding thermal fireball $[67,68]$. The first peak is the vector mass close to $T_{c}$ while the second is in the vicinity of the "zero-binding" line at $T \simeq 1.5-2 T_{c}$. The $x$-axis is in units of the quark-quasiparticle mass, $M_{q} \simeq 1 \mathrm{GeV}$. The quark-quasiparticle width is assumed to be $0.1 \mathrm{GeV}$ [36].

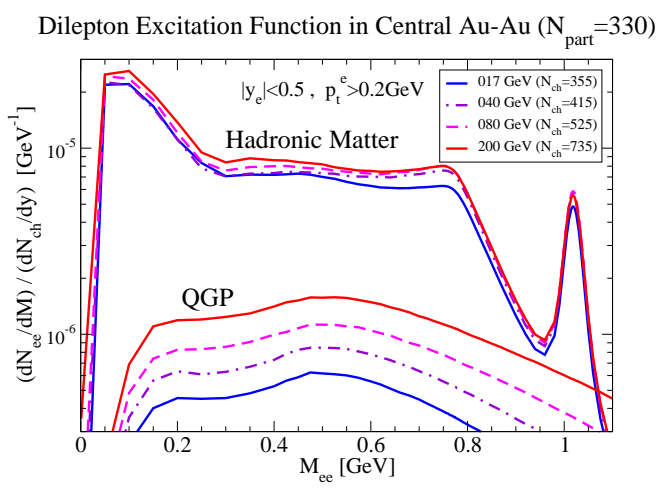

Fig. 16. Excitation function of low-mass dilepton spectra for central $\mathrm{Au}+\mathrm{Au}$ collisions from top SPS to top RHIC energy. The upper (lower) set of four curves corresponds to thermal radiation from hadronic matter (QGP) using in-medium $\rho, \omega$ and $\phi$ spectral functions $[12,68]$ (HTL $q \bar{q}$ annihilation [46]), assuming a critical temperature between $175 \mathrm{MeV}$ at $\sqrt{s_{N N}}=17 \mathrm{GeV}$ and $180 \mathrm{MeV}$ at $\sqrt{s_{N N}}>100 \mathrm{GeV}$.

doscalar ( $\pi$ " or $\eta_{c}$ ) states. The connection between these resonances and the pion mass dropping to near zero when approaching $T_{c}$ from above has been addressed in Ref. [114].

Quantitative signatures of vector resonances above $T_{c}$ in the dilepton spectrum have been evaluated in Ref. [37]. Convoluting the temperature-dependent resonance decays with an expanding fireball at RHIC $[67,68]$ predicts an enhancement over baseline pQCD emission scenario ( $q \bar{q}$ annihilation) by about a factor of $\sim 2$, see Fig. 15. This result is sensitive to the vector-meson width which in turn is governed by the width of the quark quasiparticles. Smaller widths lead to narrower peaks and thus a stronger enhancement over the pQCD spectrum. The quasiparticle width is expected to be $\sim 0.2 \mathrm{GeV}$ based on selfconsistent solutions of a $q \bar{q}$ scattering equation [36] using input interaction potentials from finite temperature lQCD or Nambu-Jona-Lasinio four-quark interactions [115]. The corresponding elastic scattering rates of $\sim 1 /(\mathrm{fm} / c)$ are suggestive of the short thermalization times deduced from hydrodynamic analyses of elliptic flow measurements and could therefore provide a link to the early thermalization puzzle at RHIC. 


\subsection{Low-Mass Dilepton Spectra}

To illustrate the predictions of medium-modified vector mesons within effective models, and in particular to investigate the importance of the baryonic component of the medium, we summarize a pertinent excitation function of low-mass dilepton spectra in central $\mathrm{Au}+\mathrm{Au}$ collisions in Fig. 16. Thermal dilepton rates in the QGP [46] and HG $[12,67]$ phases are convoluted with isentropic fireball evolution similar to that underlying Figs. 9 and 15, assuming a chemically equilibrated QGP which converts into a chemically equilibrated HG at $\left(\mu_{N}^{c}\right.$, $T_{c}$ ) values compatible with: (i) thermal models for hadron production in central $A A$ collisions [25] and (ii) a total entropy that reproduces available particle multiplicity data $[2,3,4]$. The hadronic evolution subsequent to chemical freeze-out is augmented by effective chemical potentials for hadrons that are stable under strong interactions (e.g. $\pi, K, \eta$, baryons and antibaryons). This is mandatory to maintain the observed chemical composition until thermal freeze-out [116] and, in particular, implies sizable total $(B+\bar{B})$ baryon densities in the later stages of the hadronic evolution (recall Sec. 2.5.1). Surprisingly, there is no large change in either shape or magnitude of the (hadronic) dilepton-spectrum excitation function for $20 \leq \sqrt{s_{N N}} \leq 200 \mathrm{GeV}$. The main reasons for this outcome are:

(i) Despite the large range in baryochemical potentials $\left(\mu_{B}^{c}=25-250 \mathrm{MeV}\right.$ at chemical freezeout with $T_{c}=175-180 \mathrm{MeV}$ ) and thus in net baryon density, the prevalent baryon-induced medium effects are comparable once the $B+\bar{B}$ density is properly accounted for.

(ii) The lifetime of the hadronic (and mixed) phase changes little since the larger volume expansion at higher energies is essentially compensated by an increase in radial flow inherited from the QGP phase.

This scenario should be contrasted with one where the medium effects are sensitive to the net baryon density such as simple dropping mass parameterizations, $m_{V}^{*} / m_{V}=\left(1-C \varrho_{B} / \varrho_{0}\right) \times\left(1-\left(T / T_{c}\right)^{2}\right)^{1 / n}$. In this case, a stronger variation of the excitation function is anticipated, with weaker effects at higher collision energy. QGP emission increases appreciably with $\sqrt{s}$ but remains subdominant $(\leq 20 \%)$ in the low-mass region at all energies if no significant $q_{t}$ cut is applied, as may be expected for larger initial temperatures and longer QGP lifetimes.

At masses above $\sim 1 \mathrm{GeV}$, the hadronic EM spectral function is dominated by four pion and higher contributions, encompassing annihilation reactions such as $\rho \rho, \pi \omega$, and $\pi a_{1}$. While these contributions are not included in the hadronic matter calculation shown in Fig. 16, they may become significant at $M \geq 0.9 \mathrm{GeV}$ where a similar enhancement could be related to effects of partial chiral symmetry restoration $[117,118]$. We will return to this issue in 
Sec. 2.7.2.

Finally, a few remarks on models of the space-time evolution are in order since these provide crucial input on the thermodynamic parameters for the equilibrium EM emission rates. Hydrodynamic models, if applicable, are the approach of choice since they are formulated using the same variables as the thermal emission rates. The present RHIC data suggest that ideal hydrodynamics gives a good approximation of the first $\sim 5 \mathrm{fm} / c$ after thermalization, encompassing the QGP, "mixed" phases and possibly the early hot+dense hadronic liquid phase for $T \geq 150 \mathrm{MeV}$. For lower temperatures, however, viscosity effects are expected to become significant [119]. It is presently not clear how finite viscosity affects calculations of dilepton and photon emission. The underlying uncertainties must be scrutinized, especially since the low-mass and low-momentum spectra receive significant contributions from later stages. One can either implement viscosity effects in hydrodynamics to retain the notion of thermodynamic variables or switch to transport theory $[120,121,122]$. In the latter option, it is nontrivial to properly implement broad resonances [123]. Alternatively, local temperatures and (baryon) densities could be extracted from transport simulations and convoluted with the equilibrium EM emission rates. The relative agreement of this method with viscous hydrodynamics could provide an estimate of the uncertainties in the integrated EM spectra and used to better calibrate fireball models, which are suitable parametrizations of microscopic evolution. Such comparisons will become particularly relevant if less penetrating probes, e.g. $\pi \pi$ or $\pi \gamma$ invariant-mass spectra, are calculated, as discussed later. Transport-based approaches are mandatory for thermal freezeout to quantitatively account for finite sizes, lifetimes and mean-free paths. In the late 1990s, the agreement of hydrodynamic [124], transport [125] and fireball models [12,126] with CERES low-mass dileptons [10] at the SPS has been reasonable, albeit with somewhat limited theoretical and experimental precision.

\subsection{Chiral Symmetry Restoration}

\subsubsection{Direct and Indirect Approach}

We now address the question of how, in principle, in-medium effects detected in dilepton spectra can be used to draw conclusions about chiral symmetry restoration $(\chi \mathrm{SR})$. An unambiguous consequence of the $\chi \mathrm{SR}$ is that isovector, vector and axial vector correlation functions, which are very different in the vacuum, become degenerate at and beyond the chiral transition. The question is how this happens, see Fig. 17 for an illustration. We reiterate that the effects of chiral symmetry breaking are concentrated at low masses since, already in vacuum, the correlators become degenerate in the pQCD regime. 

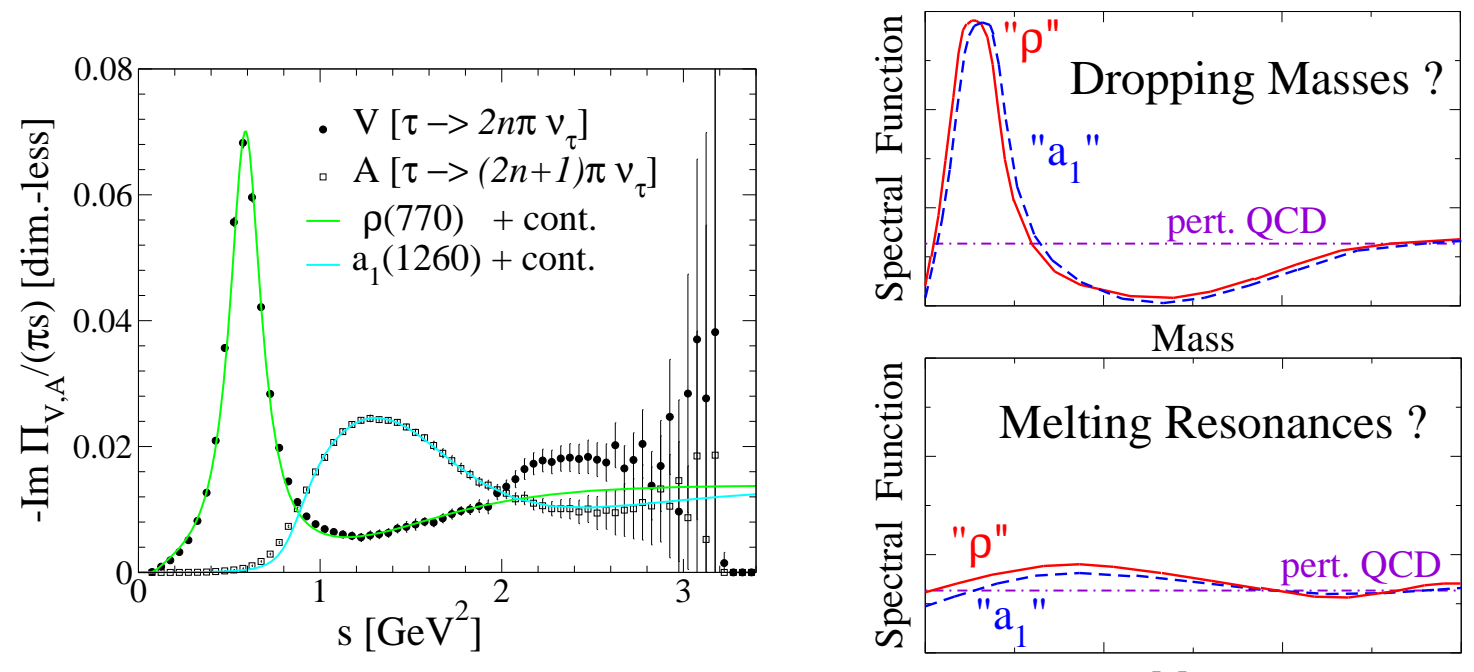

Mass

Fig. 17. Left: isovector, vector and axial vector spectral functions in the vacuum as measured in hadronic $\tau$ decays [127] with model fits using $\rho$ and $a_{1}$ resonances plus a perturbative continuum [128]. Right: two schematic scenarios of chiral symmetry restoration in hot and dense matter.

Therefore, symmetry breaking constitutes an inherently nonperturbative phenomenon which, ideally, can be addressed with input from experiment, theoretical models and lattice QCD computations.

A direct way to search for $\chi \mathrm{SR}$ is measurement of the in-medium axial vector spectral function in connection with model comparisons, as also done for dileptons. Measurements of the $\pi^{ \pm} \gamma$ invariant-mass spectra were suggested [129] as a means of searching for $\chi \mathrm{SR}$. This was partly motivated by similar measurements of $\pi^{+} \pi^{-}$invariant-mass spectra in heavy-ion collisions which indicated $\rho$ modifications in the late stages of peripheral $200 \mathrm{GeV} \mathrm{Au}+\mathrm{Au}$ collisions at RHIC [130]. Absorption effects on the outgoing pions limit the $\pi \pi$ information to rather dilute stages while $\pi \gamma$ spectra may probe somewhat deeper into the fireball. However, emission from later collision stages is advantageous since a narrower density and temperature window is probed so that the convolution over the space-time history becomes less of an issue. Experimentally the challenges are the rather low rate 11 and the rather broad structure of the $a_{1}(1260)$ resonance $(\sim 0.4 \mathrm{GeV}$ in vacuum), making it susceptible to distortions due to background subtractions. Simulations will be shown in Sec. 4.2.2.

In addition to the direct experimental approach to the axial vector channel, we now formulate a well-defined theoretical procedure based on chiral hadronic models to connect experimental information on the vector correla-

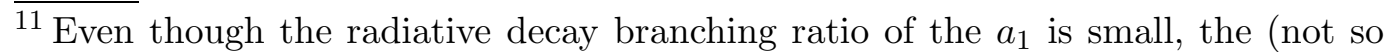
small) absolute decay width, $\Gamma_{a_{1} \rightarrow \pi \gamma} \simeq 0.7 \mathrm{MeV}$, is the relevant quantity for thermal radiation.
} 
tor (dileptons) to (first-principle) information on $\chi \mathrm{SR}$ from lattice QCD. The in-medium versions of the chiral sum rules, Eqs. (12), (13) and (14), critical to the calculation, are obtained as follows:

(1) First, calculate the vector $(V)$ and axial vector $(A)$ spectral functions as a function of temperature and density in a chirally invariant model, including as many of the constraints as possible (see Sec. 2.5.1).

(2) Then insert the spectral functions into the Weinberg sum rules to evaluate the temperature dependence of the pion decay constant and four-quark condensate and compare to lattice QCD results. Note that $f_{\pi}(T)$ and $\left\langle(\bar{q} q)^{2}\right\rangle(T)$ are presumably more easily evaluated in lQCD than spectral functions since lQCD is primarily applicable to the finite temperature axis, i.e., at $\mu_{q}=0$, the closest relation between 1QCD and heavy-ion experiments is realized in the central rapidity regions at RHIC and the LHC.

(3) Finally, perform detailed comparisons of the in-medium effects on the vector correlator with dilepton data as a function of centrality, $\sqrt{s_{N N}}$, mass and $q_{t^{-}}$ spectra. The comparison requires additional input from realistic expansion models (e.g. hydrodynamical and transport simulations), which have been thoroughly tested against the large body of hadronic observables.

The three different energy moments of $\rho_{V}-\rho_{A}$ probed by the chiral sum rules, provide detailed constraints on the energy dependence of the in-medium spectral functions. In addition, each in-medium chiral sum is valid for a given three-momentum, providing further kinematic information. Therefore, if a chiral hadronic model complies with both theoretical (2) and experimental (3) tests, a tight connection between lattice QCD and data has been established, producing explicit evidence for chiral symmetry restoration without a direct measurement of the axial vector correlator. In the absence of (unquenched) lattice data for (low-mass) spectral functions for at least the next ten years, a systematic approach involving effective models is the only way to interpret data in terms of $\chi S R$. Experimental guidance is crucial for progress in understanding the underlying nonperturbative physics.

\subsubsection{LMR-IMR Transition: Chiral Mixing}

In a low-temperature pion gas, the expectation values of vector and axial vector correlators can be evaluated model-independently based on chiral reduction formulae in connection with a low-density expansion. The leading medium effect has first been derived in Ref. [131] in the chiral limit $\left(m_{\pi}=m_{u, d}=0\right)$ and amounts to chiral correlator mixing,

$$
\begin{aligned}
& \Pi_{V}(q)=(1-\epsilon) \Pi_{V}^{\mathrm{vac}}(q)+\epsilon \Pi_{A}^{\mathrm{vac}}(q) \\
& \Pi_{A}(q)=(1-\epsilon) \Pi_{A}^{\mathrm{vac}}(q)+\epsilon \Pi_{V}^{\mathrm{vac}}(q)
\end{aligned}
$$



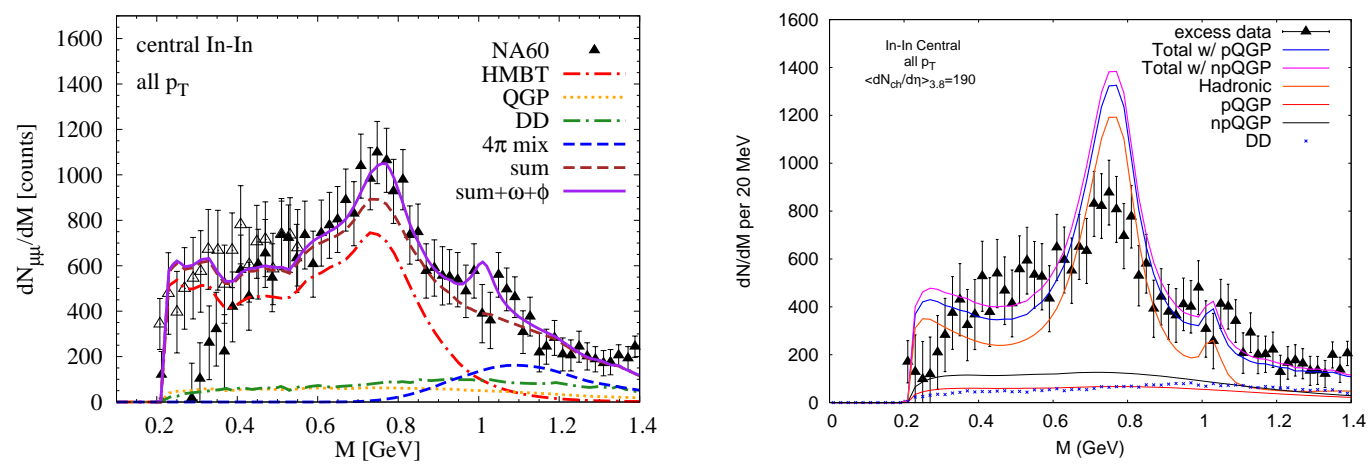

Fig. 18. Comparison of NA60 dimuon data $[6,7]$ in central In $(158 \mathrm{AGeV})+$ In collisions with calculations. Left: an expanding fireball calculation $[117,118]$ for QGP and hadronic emission. The hadronic emission is based on in-medium $\rho$ [12], $\omega[67]$ and $\phi$ spectral functions as well as four-pion contributions with an enhancement from chiral mixing according to Eq. (17). Right: hydrodymanic convolution [132] of the dilepton rates following from the chiral virial expansion $[94,107]$.

where $\epsilon=T^{2} / 6 f_{\pi}^{2}$ encodes the thermal pion density (smaller for $m_{\pi}>0$ ). Interactions of the vector current with pions from the heat bath quench the vacuum vector correlator 12 and its admixture of the axial vector correlator induced by $V+\pi \rightarrow A$ and $A+\pi \rightarrow V$ processes. The axial vector current is analogously affected. Full mixing corresponds to $\epsilon=1 / 2$, implying degenerate correlators. Broadening and possible mass shifts of the $\rho$ are thus due to higher-order effects in both $T$ and $\varrho_{N}$. The mixing has the interesting feature of filling in the "dip" in the $s=1-2 \mathrm{GeV}^{2}$ region of the vacuum vector correlator 13 (see the left panel of Fig. 17). Dilepton enhancement by up to a factor of 2 over the vacuum vector spectral function in this mass region is therefore a signature of the approach to chiral restoration, via $\pi a_{1}$ annihilation (four-pion contributions not present in the vacuum EM correlator) or QGP emission. While the earlier SPS dilepton data did not have the necessary precision for the required quantitative analysis (recall Fig. 2), it may be feasible with the new NA60 data [6,7] shown in the left panel of Fig. 18. The blue dashed curve, a theoretical upper estimate [118], employs Eq. (17) with $\epsilon(T)=\frac{1}{2} n_{\pi}(T) / n_{\pi}\left(T_{c}\right)$ where $n_{\pi}(T)$ is the pion density at $T \leq T_{c}$, including pion chemical potentials below $T_{c}=175 \mathrm{MeV}$, and removing the $a_{1} \rightarrow \pi \rho$ decay, included in the $\rho$ in-medium spectral function. On one hand, it is gratifying to see that this calculation properly accounts for the excess spectrum in the relevant mixing regime. On the other hand, the data are still reasonably well described without mixing. To be sensitive to the mixing effect, both the data and the theory require an accuracy of at least $20 \%$. The chiral virial ap-

\footnotetext{
$\overline{12}$ The shape of the correlator is unaffected.

${ }^{13}$ Full mixing in this regime leads to degenerate $V$ and $A$ correlators that closely coincide with the pQCD $q \bar{q}$ continuum level, interpreted as lowering the "duality scale" from $s \simeq 2.5 \mathrm{GeV}^{2}$ in the vacuum to about $1 \mathrm{GeV}^{2}$ for full mixing. It is further tempting to interpret the $\rho$ melting found in hadronic many-body theory (recall Sec. 2.5.1) as lowering the duality scale as $s \rightarrow 0$, implying chiral restoration [105].
} 

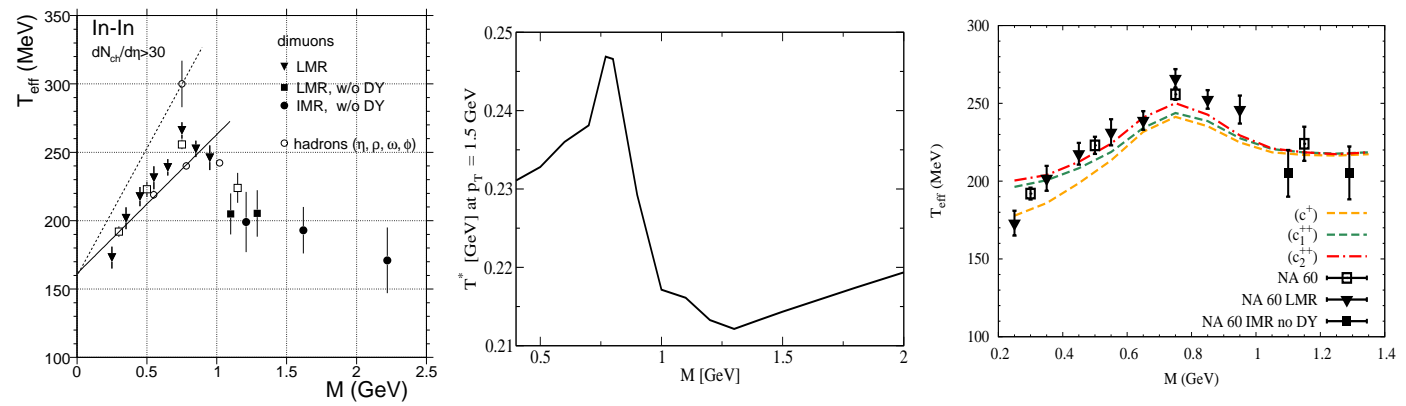

Fig. 19. Effective slope parameters as a function of dimuon invariant mass for excess radiation in In $(158 \mathrm{AGeV})+\mathrm{In}$ collisions at the SPS. Note the different vertical and horizontal scales on each plot. Left: experimental results with $d N_{\mathrm{ch}} / d y>30$ summed over all centralities and fit over $0.4<q_{t}<1.8 \mathrm{GeV} / c$ [135]. Center: fireball calculations of semicentral collisions based on thermal emission plus free $\rho$ decays at freezeout using the local slope at $q_{t}=1.5 \mathrm{GeV}$ [136]. Right: fireball calculations of semicentral collisions based on thermal and non-thermal sources fit over $1.0<q_{t}<1.8 \mathrm{GeV}$. The lower dashed line is a baseline calculations while the upper dashed and dot-dashed lines also include $t$-channel meson exchange in $\pi \rho \rightarrow \pi \mu \mu$ reactions [106].

proach, when folded over hydrodynamic evolution, as shown in the right panel of Fig. 18, also describes the region $M \geq 1 \mathrm{GeV}$ well [132]. The free EM correlator with mixing effects is a key ingredient, resulting in an enhancement consistent with maximal mixing as the upper limit and the free EM correlator as the lower limit. Although the $\rho$ peak is quenched (Sec. 2.5.1), the abscence of $\rho$ resonance broadening results in a $\sim 40 \%$ overestimate of the yield around the free $\rho$ mass. The enhancement below the $\rho$ mass is again accounted for, with important baryon contributions. In both approaches underlying Fig. 18, the QGP yield is small. The conclusions of Ref. [118] on $\rho$ broadening and the importance of baryon-driven medium effects in the context of the NA60 data have been confirmed in Ref. [133] which, however, attributes the bulk of the enhancement above the $\rho$ mass to QGP radiation. The relative QGP to four-pion yields in the IMR is essentially determined by the choice of $T_{c}$ in the fireball evolution [106]: QGP dominates the IMR if $T_{c}=160 \mathrm{MeV}$ while hadronic contributions are dominant if $T_{c}=175 \mathrm{MeV}$. Irrespective of whether the source is of QGP or hadronic origin, the IMR enhancement is associated with matter at temperatures close to $T_{c}$. The robustness of this conclusion is again a consequence of "parton-hadron duality" in the underlying emission rates.

Additional information on the nature of the emitting source might be obtained from quantitative analysis of dilepton $q_{t}$ spectra, including elliptic flow [134]. Such analysis has become possible with the NA60 data [135]. Effective inverse slope parameters, $T_{\text {eff }}$, have been extracted from dilepton excess spectra in the momentum range $0.4<q_{t}<1.8 \mathrm{GeV}$, obtained from inclusive $\mathrm{In}+\mathrm{In}$ collisions at the SPS with $d N_{\mathrm{ch}} / d y>30$. The resulting $T_{\text {eff }}$ are shown as a function of 
dilepton invariant mass on the left side of Fig. 19. The extracted values of $T_{\text {eff }}$ appear quite large relative to the hadronic slopes at freezeout, requiring a surprisingly strong collective flow for the moderate system size at SPS energies. The maximum $T_{\text {eff }}(M)$, in the free $\rho$ mass region, is indicative of $\rho$ decays in the late stages of the collision where the line shape is expected to approach the vacuum shape while the effective temperature, $T_{\text {eff }} \simeq T_{\mathrm{fo}}+m \bar{v}^{2}$, is primarily due to the blue shift imprinted by the collective expansion velocity, $\bar{v}$. Note that the slope of the excess radiation at $M=m_{\rho, \omega}$ is larger than that of the $\omega$ (lower circle at $M=m_{\rho, \omega}$, suggesting that the $\rho$ freezes out significantly later than the $\eta, \omega$ and $\phi$ (the upper open circle at $M=m_{\rho, \omega}$, estimated for $\rho$ decays around freezeout). In the IMR, $T_{\text {eff }}$ is significantly reduced, in agreement with thermal emission early in the evolution, from temperatures close to $T_{c}$ where the flow has not yet developed much, implying a small blue shift. Quantitative theoretical analyses $[106,136]$ are rather involved, see the center and right panels of Fig. 19, especially for momenta above $q_{t} \simeq 1-1.5 \mathrm{GeV}$ where nonthermal sources are expected to become significant [137], e.g., Drell-Yan dileptons or primordial $\rho$ decays.

It would be very valuable to obtain similar information for $\mathrm{Au}+\mathrm{Au}$ collisions at RHIC. The thermal yield is expected dominate further, contributing at higher momenta, due to the larger system size and collision energy 14 . Unfortunately, the increased contribution from correlated charm decays is sensitive not only to the total charm cross section but also to the charm momentum spectra and thus to charm thermalization (at low and intermediate $p_{T}$ ) and energy loss (at high $p_{T}$ ). However, open heavy flavor spectra are interesting independent probes of the medium and their measurement therefore constitutes one of the central goals of RHIC-II, see the heavy flavor part of this report. It would also be very illuminating to check whether the much increased partonic collectivity at RHIC reflects itself by an increased $T_{\text {eff }}$ in the IMR 15 .

\subsection{Electromagnetic Signatures of the Color Glass Condensate}

Saturation physics has been applied to the description of RHIC data quite successfully, from hadron multiplicities and the phenomenon of limiting fragmentation in $\mathrm{Au}+\mathrm{Au}$ and $\mathrm{d}+\mathrm{Au}$ collisions to the produced hadron transversemomentum spectra in $\mathrm{d}+\mathrm{Au}$ collisions at mid- and forward rapidity (for recent reviews and an extensive list of references, see [138]). Nevertheless, in order to establish gluon saturation as the dominant physics responsible for these phenomena at RHIC and beyond, and to rule out other phenomenological scenarios, one needs to consider further tests of the CGC formalism such as

${ }^{14}$ The multiplicity, $d N_{\mathrm{ch}} / d y$ in $\mathrm{Au}+\mathrm{Au}$ collisions at RHIC is about a factor of four larger than that of In+In collisions at the SPS.

${ }^{15}$ We thank $\mathrm{Nu} \mathrm{Xu}$ for an interesting discussion on this point. 


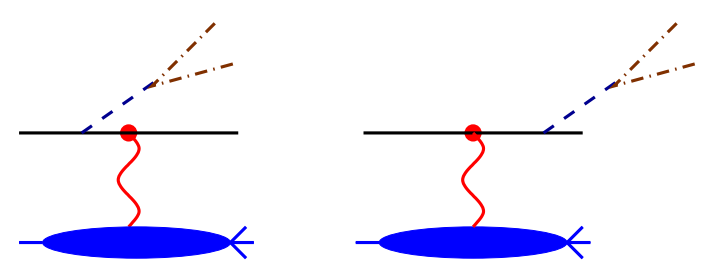

Fig. 20. Dilepton production in quark-nucleus scattering.

the predictions of saturation physics for electromagnetic processes. In this section we outline electromagnetic signatures of the CGC at RHIC. Specifically, photon and dilepton production in $\mathrm{d}+\mathrm{Au}$ collisions are considered. These processes have an advantage over hadronic processes in that they do not interact strongly after they are produced and the nonperturbative hadronization process is absent. Furthermore, photon and dilepton production can shed light on the validity of the recombination model approach to hadron production which are also capable of fitting the available data, albeit with a few assumptions. Since saturation physics predicts similar suppression patterns for photon, dilepton and hadron production in $d+A u$ collisions, an experimental confirmation of this generic prediction would be a major step in establishing saturation physics at $\mathrm{RHIC}$ and in ruling out recombination as the physics of hadron production in $\mathrm{d}+\mathrm{Au}$ collisions.

\subsubsection{Dilepton and Photon Production}

We consider the dilepton-production cross section in quark-nucleus scattering [139]

$$
q(p)+A \rightarrow q(q)+l^{+}\left(k_{1}\right)+l^{-}\left(k_{2}\right)+X
$$

shown in Fig. 20, where $k_{1}$ and $k_{2}$ are the momenta of the two leptons. Photon production can be obtained by taking the photon virtuality (dilepton invariant-mass) to zero. The differential cross section at fixed impact parameter, $b_{t}$, is given by

$$
\begin{aligned}
& \frac{d \sigma^{q A \rightarrow q l^{+} l^{-} X}}{d^{2} b_{t} d^{2} k_{t} d \ln M^{2} d z}=\frac{2 e_{q}^{2} \alpha^{2}}{3 \pi} \int \frac{d^{2} q}{(2 \pi)^{4}} \sigma_{\text {dipole }}^{F}\left(l_{t}, b_{t}, x_{A}\right) \\
& \left\{\left[\frac{1+(1-z)^{2}}{z}\right] \frac{z^{2} l_{t}^{2}}{\left[k_{t}^{2}+M^{2}(1-z)\right]\left[\left(k_{t}-z l_{t}\right)^{2}+M^{2}(1-z)\right]}\right. \\
& \left.-z(1-z) M^{2}\left[\frac{1}{\left[k_{t}^{2}+M^{2}(1-z)\right]}-\frac{1}{\left[\left(k_{t}-z l_{t}\right)^{2}+M^{2}(1-z)\right]}\right]^{2}\right\}
\end{aligned}
$$

where $z$ is the fraction of the incoming quark light cone energy carried away by the (virtual) photon while $x_{A}$ is the Bjorken $x$ probed in the target nucleus. 
All quark masses are ignored, $M^{2}$ is the dilepton invariant mass squared with $l_{t}=q_{t}+k_{t}$ and $k_{t}$ is the transverse momentum of the lepton pair.

Eq. (19) is the standard expression obtained if propagation of the incident quark through the nucleus is assumed to be eikonal, i.e., the transverse momentum transferred to the nucleus by the incident quark is much less than its longitudinal momentum [140]. All the information on the degrees of freedom in the target is contained in the dipole cross section, $\sigma_{\text {dipole }}^{F}\left(b_{t}, l_{t}, x_{A}\right)$. Gluon saturation physics comes in via the dipole cross section, which is determined by the evolution equations of the Color Glass Condensate. Given an initial condition for the dipole cross section, the JIMWLK equations in the Color Glass Condensate formalism determine the dependence of the dipole cross section on the collision energy (or alternatively, $x_{A}$ ) and $l_{t}$. This is the main difference between the CGC formalism and the other approaches [140]: while the CGC formalism can predict the $x_{A}$ and $l_{t}$ dependence of the dipole cross section, other approaches cannot and must instead motivate a suitable form from phenomenological considerations. We note that to obtain the invariant cross section for photon production, $d \sigma^{q A \rightarrow q \gamma X} / d^{2} b_{t} d^{2} k_{t} d z$, from Eq. (19), the mass is set to zero, $M=0$, and the dilepton vertex factor, $\alpha / 3 \pi M^{2}$, is removed.

Since the dipole cross section is also the main ingredient in the hadron production cross sections in deuteron (proton)-nucleus collisions, a similar nuclear modification factor, $R_{d A}$, is predicted for dilepton production and hadron production. Parametrically, one expects the nuclear modification factor, $R_{p A}$, to scale with the nucleon number $A$ like $R_{p A} \sim A^{-\frac{\gamma_{0}}{3}}$ where $1-\gamma_{0} \simeq 0.628$ is the BFKL anomalous dimension. It should be emphasized that in QCD, small$x$ evolution (BFKL) is the only way to generate leading-twist shadowing if partonic degrees of freedom (quarks and gluons) are used.

Dilepton production has an additional knob to turn in order to change the kinematics and probe QCD dynamics in different settings, the dilepton invariant mass. The nuclear modification factor, calculated using a saturation inspired model of the dipole cross section [141], is shown in Fig. 21.

Since the dilepton production rates are small due to the electromagnetic coupling, we consider the invariant-mass dependence of the transverse-momentum integrated cross section. The integration over Eq. (19) can be done analytically to obtain

$$
\begin{aligned}
& z \frac{d \sigma^{q A \rightarrow q l^{+} l^{-} X}}{d^{2} b d M^{2} d z}=\frac{\alpha^{2}}{3 \pi^{2}} \frac{1-z}{z^{2}} \int d r_{T}^{2} \sigma_{\text {dipole }}^{F}\left(x_{g}, \underline{b}, r_{T}\right) \\
& \left.\quad\left[1+(1-z)^{2}\right] K_{1}^{2}\left[\frac{\sqrt{1-z}}{z} M r_{T}\right]+2(1-z) K_{0}^{2}\left[\frac{\sqrt{1-z}}{z} M r_{T}\right]\right] .
\end{aligned}
$$




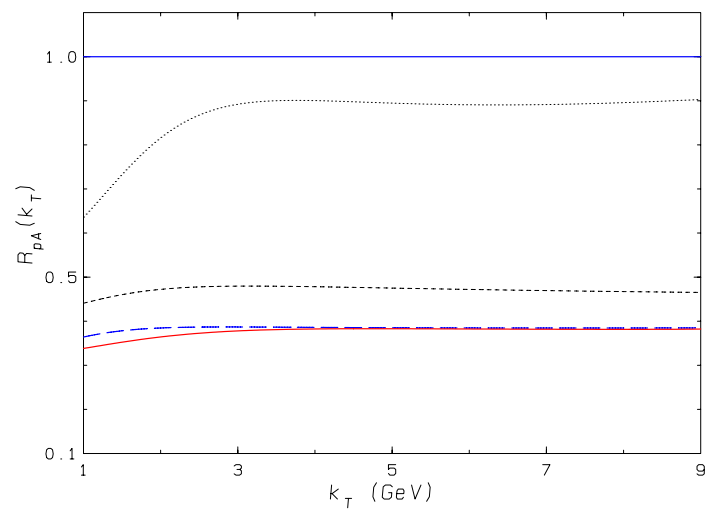

Fig. 21. Nuclear modification factor, $R_{d A}$, of dilepton production in quark-nucleus scattering [141]. The dotted, short-dashed and long-dashed curves are for $M=2$ $\mathrm{GeV}$ and $y=0.5,1.5$ and 3 respectively. The solid curve is for $M=4 \mathrm{GeV}$ and $y=3$.

To relate this to proton (deuteron)-nucleus scattering, Eq. (20) must be convoluted with the quark (and antiquark) distributions, $q\left(x, M^{2}\right)\left(\bar{q}\left(x, M^{2}\right)\right)$ in a proton or deuteron. This can be written in terms of the proton (deuteron) structure function $F_{2}$ [140],

$$
\begin{array}{r}
\frac{d \sigma^{p A \rightarrow l^{+} l^{-} X}}{d^{2} b d M^{2} d x_{F}}=\frac{\alpha^{2}}{6 \pi^{2}} \frac{1}{x_{q}+x_{g}} \int_{x_{q}}^{1} d z \int d r_{T}^{2} \frac{1-z}{z^{2}} F_{2}^{p}\left(x_{q} / z\right) \sigma_{\text {dipole }}^{F}\left(x_{g}, \underline{b}, r_{T}\right) \\
{\left[\left[1+(1-z)^{2}\right] K_{1}^{2}\left[\frac{\sqrt{1-z}}{z} M r_{T}\right]+2(1-z) K_{0}^{2}\left[\frac{\sqrt{1-z}}{z} M(q 2]\right]\right]}
\end{array}
$$

where

$$
\begin{aligned}
& x_{q}=\frac{1}{2}\left[\sqrt{x_{F}^{2}+\frac{4 M^{2}}{s}}+x_{F}\right], \\
& x_{g}=\frac{1}{2}\left[\sqrt{x_{F}^{2}+\frac{4 M^{2}}{s}}-x_{F}\right], \\
& x_{F} \equiv \frac{M}{\sqrt{s}}\left[e^{y}-e^{-y}\right],
\end{aligned}
$$

and

$$
F_{2}^{p} \equiv \sum_{f} e_{f}^{2} x\left[q_{f}\left(x, M^{2}\right)+\bar{q}_{f}\left(x, M^{2}\right)\right]
$$

is the proton structure function.

The invariant dilepton production cross section is shown in Fig. 22 for protonproton, proton-nucleus and deuteron-nucleus collisions as a function of dilep- 


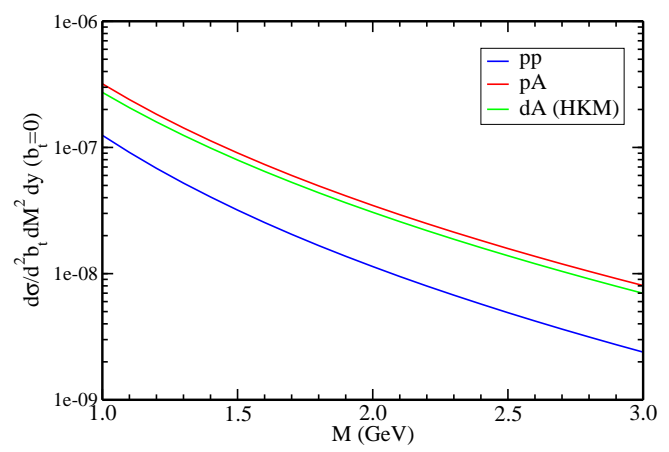

Fig. 22. Dilepton production cross section at $y=2.2$.

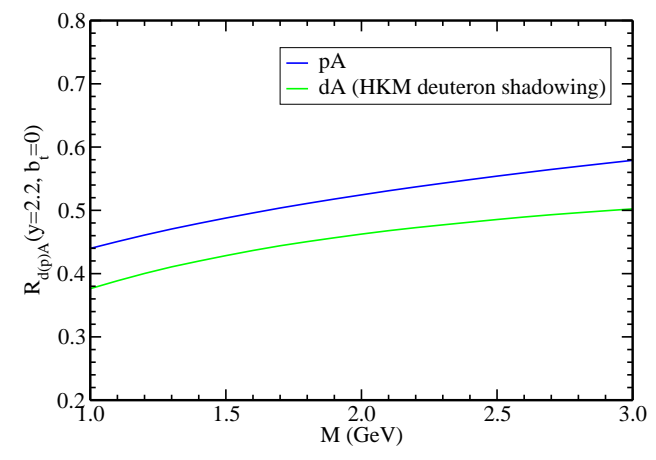

Fig. 23. The dilepton nuclear modification factor in $p A$ and $\mathrm{d} A$ collisions at $y=2.2$.

ton invariant mass, while Fig. 23 shows the nuclear modification factor for both $p A$ and $\mathrm{d} A$ collisions at RHIC. The calculations are for fixed rapidity, $y=2.2$, and the most central collisions [142]. The HKM parameterization of the deuteron wave function is used for the deuteron projectile, a few percent effect.

Photon production and photon and hadron correlation functions have been calculated using the same formalism [142]. The hadron-photon cross section is a very sensitive probe of the dipole profile, the main ingredient of singleparticle production cross section in the CGC formalism. Therefore, experimental studies of electromagnetic probes in deuteron-nucleus collisions at RHIC can shed light both on the dynamics of gluon saturation and on the role of saturation physics in the observed suppression of the hadron spectra in the forward rapidity region at RHIC. This measurement can also clarify the role of hadron recombination models at RHIC, at least in the forward rapidity region, since recombination effects will not be present in electromagnetic final states. Therefore, observation of dilepton or photon suppression would be strong evidence for the CGC.

It is worth noting that, since the saturation scale of the proton (deuteron) is very small at mid- and forward rapidity at RHIC, CGC predictions for protonproton collisions will have large uncertainties. Indeed, as recently shown [143], particle production in proton-proton collisions cannot be reliably calculated at RHIC, since the saturation momentum is small, while $d+A u$ calculations are under much better quantitative control. Therefore, in any saturation-inspired calculation of the nuclear modification factor, the $p p$ cross section in the denominator should be understood to be a fit to the data while the $\mathrm{d}+\mathrm{Au}$ cross section can be calculated using saturation physics and used to determine the physics dominant in the forward-rapidity region at RHIC. This, in turn, will have significant ramifications for the LHC. 


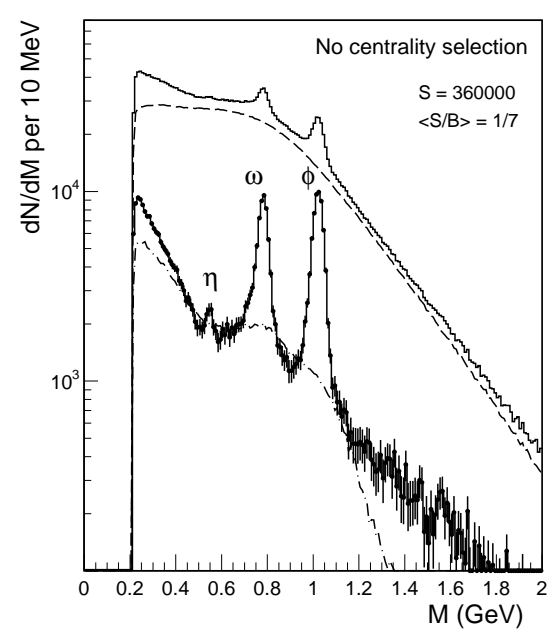

Fig. 24. Dimuon invariant-mass spectra from NA60 [6,7]. The raw data (upper histogram) show clear $\omega$ and $\phi$ peaks. In the background-subtracted spectrum, (lower histogram) the $\eta \rightarrow \mu \mu$ decay is recognizable.

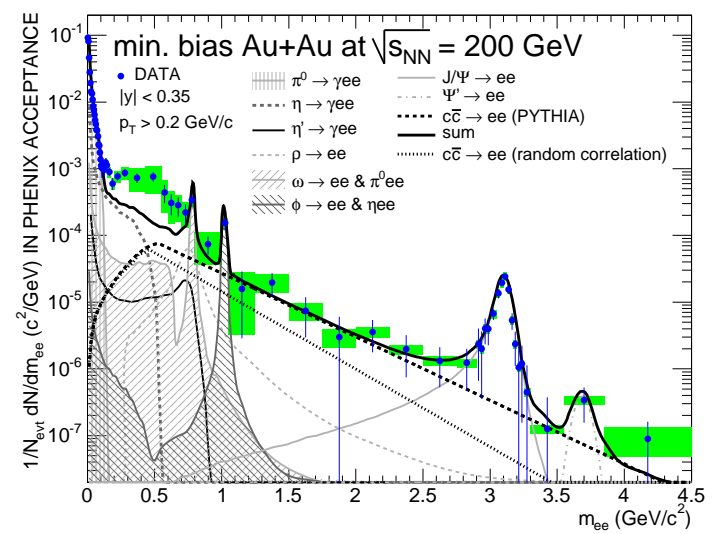

Fig. 25. Dilepton mass spectrum from PHENIX [146] compared to a cocktail of hadronic sources and charm decays.

\section{OBSERVABLES: STATE OF THE ART}

\subsection{Low-Mass Dileptons}

The $\rho$ meson, with its short lifetime $(1.3 \mathrm{fm} / c$ in vacuum), large dilepton decay width $\left(\Gamma_{e e}=7 \mathrm{keV}\right.$ ) and prevalent coupling to $\pi \pi$ (and possibly $q \bar{q}$ ) annihilation has long been identified as the most promising probe of in-medium modifications of hadron properties close to the QGP phase boundary. Changes in $\rho$ mass or width were anticipated as precursors of the chiral transition more than two decades ago [144]. Dielectron mass spectra from CERES [10,22] over the last ten years have shown a significant (factor of $2-3$ ) excess over known hadronic sources in the mass region below the free $\rho$ mass - a result which spurred vigorous theoretical activity. However, the CERES experimental uncertainties, including limited statistics and mass resolution, could neither confirm nor refute scenarios with medium effect: 16. Recently, the NA60 experiment at the SPS, a significant upgrade of NA50 including high-precision tracking close to the vertex in a high magnetic field, measured dimuon mass spectra in $158 \mathrm{AGeV}$ In+In collisions with an unprecedented $\sim 20 \mathrm{MeV}$ mass resolution and high statistics in both the low and intermediate mass regions $[6,7]$. The results in the light vector-meson region are shown in Fig. 24. After subtracting the "hadron decay cocktail" with $\eta, \omega$, and $\phi$ decays, the excess

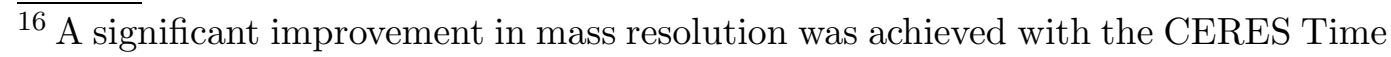
Projection Chamber upgrade [145]. 
spectrum has a clear albeit rather broad peak around the nominal $\rho$ mass, as shown in Fig. 18 and compared to theoretical predictions using in-medium $\rho$ spectral functions with either a dropping mass [11] or a strong broadening, as calculated in hadronic many-body theory [12]. The excellent resolution and statistics, made possible by detector upgrades and high luminosity, can now clearly distinguish between these two approaches and be considered as a benchmark for future dilepton measurements. (How well other approaches can explain the measured data remains to be seen.) Measurements of similar precision - made possible by RHIC-II - will be essential to further improve our understanding and elevate it from the qualitative to the quantitative level, necessary for drawing conclusions about the nature of chiral symmetry restoration.

Comparable quality measurements of the $\rho$ spectral function do not yet exist at RHIC. PHENIX is measuring dielectrons in the central arm. The mass spectrum in minimum bias $200 \mathrm{GeV} \mathrm{Au}+\mathrm{Au}$ collisions is shown in Fig. 25 along with the hadronic cocktail and the background from charm. While the $J / \psi$ measurement is very clean, the resolution in the low mass region is not adequate to test in-medium modifications of vector mesons. Such precision is, however, expected once the hadron-blind detector, one of the major PHENIX upgrades becomes operational (see Sec. 4.1.2).

STAR measured the $\pi^{+} \pi^{-}$invariant-mass spectra [130] and found indications that the $\rho$ mass is both $p_{T}$ and multiplicity-dependent in $p p, \mathrm{~d}+\mathrm{Au}$ and peripheral $\mathrm{Au}+\mathrm{Au}$ collisions. At low transverse momenta, $0.5<p_{T}<1.5 \mathrm{GeV} / c$, the $\rho$ mass peak is $3-8 \%$ lower. Whether this is caused by a $\rho$ mass shift $[147,148]$ or other effects (e.g. Bose-Einstein correlations, thermal phase space in connection with resonance broadening, underlying " $\sigma$ " decays, etc. $[129,149])$ still needs to be clarified. The measurement was insensitive to possible changes in the $\rho$ width [150] which requires an improved determination of the background sources including the "physics background" 17 .

The combination of leptonic and hadronic $\rho$ decays is a valuable tool for disentangling in-medium modifications due to the hot and dense phases from the more dilute phases. The leptonic decays probe the entire collision history while the hadronic decays probe the fireball surface and the late stages.

\subsection{The $\phi(1020):$ Hadronic vs Leptonic Decays and $v_{2}$}

Since the $\phi$ mass is barely twice the kaon mass, $m_{\phi}-2 m_{K} \simeq 30 \mathrm{MeV}$, a study of hadronic, $\phi \rightarrow K \bar{K}$, relative to leptonic, $\phi \rightarrow e^{+} e^{-}$, decays has long

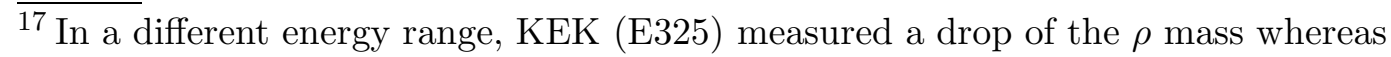
JLAB (CLAS) reported broadening without any drop of the $\rho$ mass. 


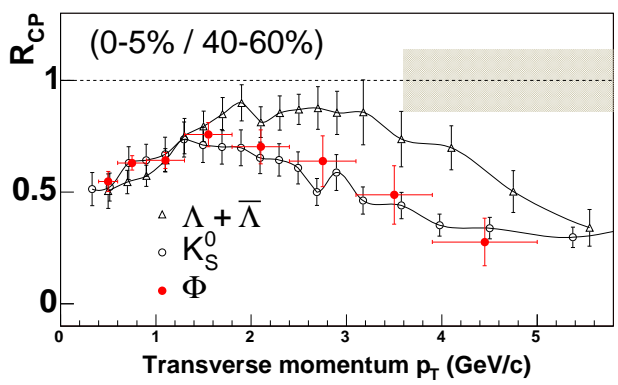

Fig. 26. The nuclear modification factor $R_{C P}$ (ratio of yields in central and peripheral collisions, divided by the respective number of binary $N N$ collisions) for $\phi, K_{s}^{0}$ and $\Lambda$ in $200 \mathrm{GeV}$ $\mathrm{Au}+\mathrm{Au}$ collisions [153].

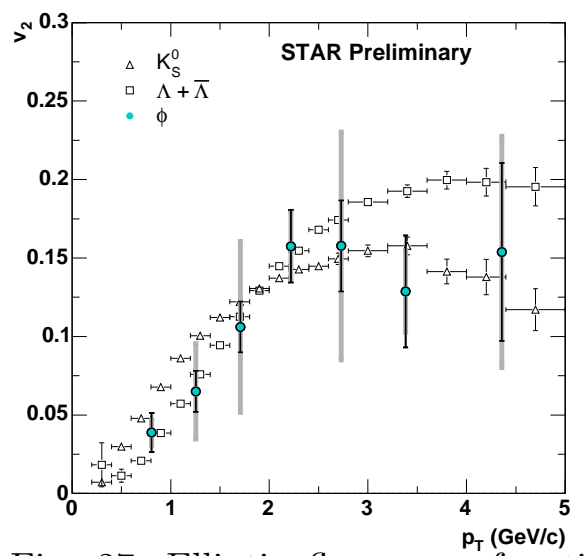

Fig. 27. Elliptic flow as a function of $p_{T}$ for $\phi, K_{s}^{0}$ and $\Lambda$ in $200 \mathrm{GeV}$ $\mathrm{Au}+\mathrm{Au}$ collisions [153].

been suggested as a sensitive test of a dropping $\phi$ mass or braodening of the $\phi$ width, possibly related to partial chiral symmetry restoration. If the mass drops, the dominant $\phi \rightarrow K \bar{K}$ channel is quickly kinematically suppressed and the ratio of production cross sections of the hadronic, $B(\phi \rightarrow K \bar{K})=49.2 \%$, and leptonic, $B\left(\phi \rightarrow e^{+} e^{-}\right)=3 \times 10^{-4}$, channels, $\sigma(\phi \rightarrow K \bar{K}) / \sigma\left(\phi \rightarrow e^{+} e^{-}\right)$, should change dramatically. Similar arguments may apply if a reduced kaon mass or inelastic scatterings such as $\phi+\pi \rightarrow K+K^{*}$ lead to a strong increase of the $\phi$ width. In this case, $\phi$ regeneration has to be accounted for as well [151]. Recent preliminary results from PHENIX [152] suggest that, when normalized by the branching ratio, $d N\left(\phi \rightarrow e^{+} e^{-}\right) / d y$ is larger and the $m_{T}$ slopes are steeper in $A A$ relative to $p p$ collisions. However, the experimental uncertainties prevent definite conclusions. The main problem is the small signal-to-background ratio $(\mathrm{S} / \mathrm{B})$ in the dielectron channel which can only be cured by better background rejection. However, the $\phi$ yields from $\phi \rightarrow K \bar{K}$ extracted by STAR [153] are systematically higher than those measured by PHENIX in the same decay channel. Careful analysis shows that $p_{T}$ spectra measured by the two experiments are not inconsistent [154].

A difference was observed between $\phi \rightarrow K \bar{K}$ measured by NA49 and $\phi \rightarrow e e$ measured by NA50. Recent CERES [155] results for both decay channels in $\mathrm{Pb}+\mathrm{Au}$ collisions and measurements of $\phi \rightarrow \mu^{+} \mu^{-}$decays in In+In collisions from NA60 [156] are consistent with data from NA49. Thus the difference was not due to the observed decay channel but the method of observation. This experience emphasizes both the importance of powerful detector upgrades and the ability to measure different decay channels in the same apparatus over a wide momentum range. 


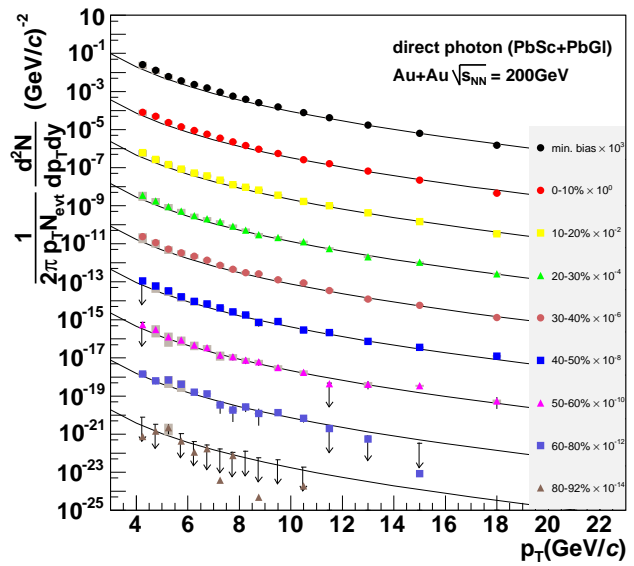

Fig. 28. Preliminary PHENIX direct photon spectra in $200 \mathrm{GeV} \mathrm{Au}+\mathrm{Au}$ collisions, for all centralities and minimum bias collisions. The curves are NLO pQCD calculations with the scale set equal to $p_{T}$.

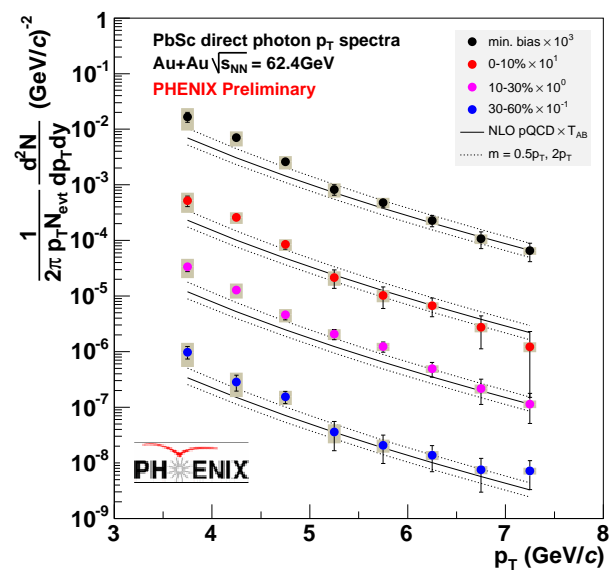

Fig. 29. Preliminary PHENIX direct photon spectra in $62 \mathrm{GeV} \mathrm{Au}+\mathrm{Au}$ collisions at different centralities compared to NLO pQCD predictions at scales of $0.5 p_{T}, p_{T}$, and $2.0 p_{T}$.

Since the $\phi$ meson mass is comparable to some baryon masses, it can also play a major role in distinguishing between the mass and species dependence of quantities such as the nuclear modification factor, $R_{C P}$, and elliptic flow. Recent results from STAR [153] in $200 \mathrm{GeV} \mathrm{Au+Au} \mathrm{collisions} \mathrm{show} \mathrm{that} \mathrm{both}$ $R_{C P}$ (Fig. 26) and $v_{2}$ (Fig. 27) are consistent with parton recombination and collective flow at the partonic level, as well as with the PHENIX data [157].

\subsection{Direct Photon Spectra}

The latest direct photon spectra from $\mathrm{Au}+\mathrm{Au}$ collisions over the full centrality range are compared to NLO pQCD calculations at $200 \mathrm{GeV}$ (Fig. 28) and $62 \mathrm{GeV}$ (Fig. 29). While the data seem to favor a perturbative scale of $0.5 p_{T}$, particularly at lower transverse momenta, they are consistent with a scale equal to $p_{T}$ at higher momenta 18 . One explanation could be the onset of additional photon sources such as photons from jet-medium interactions at intermediate $p_{T}$ (see Fig. 12). Before drawing any conclusions, high quality $p p$ data have to determine the level of agreement of the data with the NLO pQCD predictions, see Sec 3.4. Unfortunately there are different competing sources of the excess relative to baseline pQCD calculations proposed - all with their respective theoretical uncertainties. Studying inclusive cross sections is insufficient for disentangling these sources. A possible solution, involving much larger data samples than currently available, is proposed in Sec. 3.5.

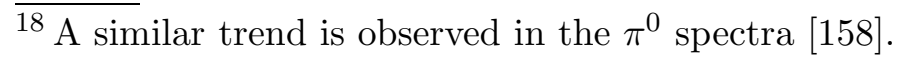




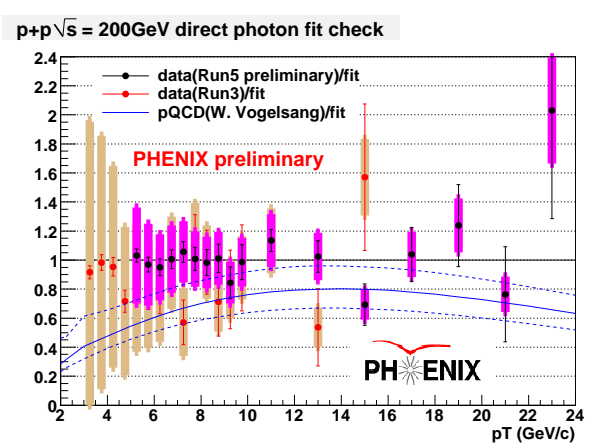

Fig. 30. Direct photons in $200 \mathrm{GeV}$ $p p$ collisions from different PHENIX runs divided by a fit to the preliminary Run-5 results. The black circles show preliminary Run-5 results to illustrate the quality of the fit while the red circles are published Run-3 data. The blue curves are NLO pQCD calculations.

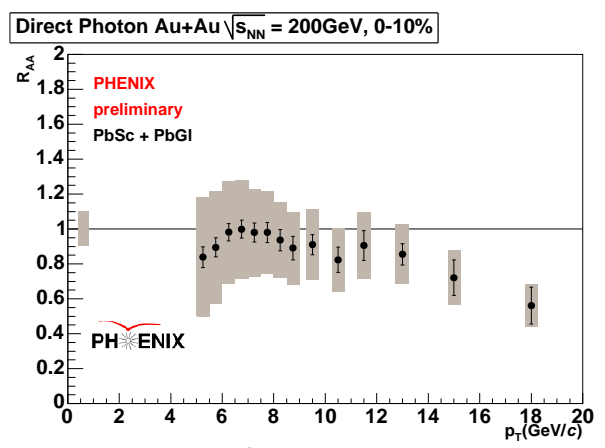

Fig. 31. Preliminary PHENIX direct photon $R_{A A}$ in the most central (0-10\%) $200 \mathrm{GeV} \mathrm{Au+Au} \mathrm{collisions,} \mathrm{fit}$ to the $p p$ direct photon spectra measured in the same experiment.

\subsection{Direct Photon $R_{A A}$}

The first RHIC measurement of the photon nuclear modification factor, shown in Fig. 1, suggests that the direct photon $R_{A A}$ is neither enhanced nor suppressed, as opposed to the observed strong hadron suppression shown on the right side of Fig. 1. The $\pi^{0}$ suppression observed in the very first RHIC $\mathrm{Au}+\mathrm{Au}$ run but absent in $\mathrm{d}+\mathrm{Au}$ collisions was interpreted as a final-state effect. Both the $\pi^{0}$ and direct photon $R_{A A}$ interpretations assume that, at high $p_{T}$ where hard scattering should dominate, scaling the $p p$ cross sections by the nuclear overlap function $T_{A B} 19$ is a sensible estimate of the expected $A A$ yields in the absence of a medium. Jets are quenched relative to this expectation. Strictly speaking, only the direct photon $R_{A A}$ proves that this expectation is reasonable: photons produced by hard scattering should leave the medium unchanged and their yield should thus scale with $T_{A B}$. This assumption has indeed been confirmed within $\sim 20-30 \%$ uncertainty by RHIC Run- 2 Au+Au data [8] and by the integrated $R_{A A}$ with $p_{T}>6 \mathrm{GeV} / c$ where $R_{A A}$ was calculated using an NLO pQCD calculation since $p p$ direct photon data were not yet available.

The much larger $\mathrm{Au}+\mathrm{Au}$ data set from Run-4, the two different collision energies $\left(\sqrt{s_{N N}}=200\right.$ and $\left.62 \mathrm{GeV}\right)$ and measurement of $p p$ reference data in the same experiment with similar systematic errors made it possible to study the evolution of $R_{A A}$ with $p_{T}$ and collision centrality in much greater detail. The fit to the $p p$ data differs both in shape and magnitude from NLO pQCD [159]. The data are higher, particularly at the lower and upper ends of the $5<p_{T}<23 \mathrm{GeV} / c$ range, see Fig. 30. The $R_{A A}$ obtained from a fit to

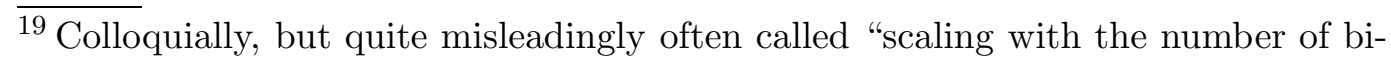
nary collisions" or $N_{\text {coll }}$ scaling 


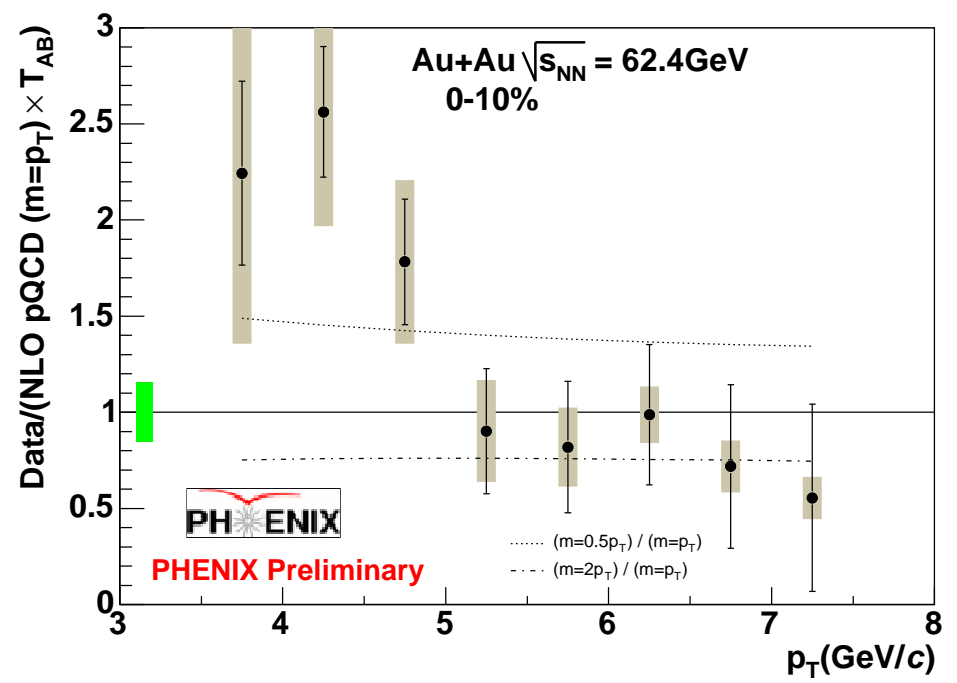

Fig. 32. Preliminary PHENIX direct photon $R_{A A}$ in the most central (0-10\%) 62 $\mathrm{GeV} \mathrm{Au+Au} \mathrm{collisions,} \mathrm{using} \mathrm{an} \mathrm{NLO} \mathrm{pQCD} \mathrm{calculation} \mathrm{with} \mathrm{scales} \mathrm{of} 0.5 p_{T}, p_{T}$, and $2.0 p_{T}$.

the $p p$ data is shown in Fig. 31 for the most central $200 \mathrm{GeV} \mathrm{Au+Au} \mathrm{colli-}$ sions. There is an apparent suppression at very high $p_{T}$. At least part of the suppression has been predicted due to the "isospin effect" [160], a natural consequence of the different quark content $\left(\sigma \propto \Sigma e_{q}^{2}\right)$ of protons and neutrons 20 . Hence the suppression in the photon $R_{A A}$ is referred to as "apparent". Other mechanisms such as modifications of the parton densities and fragmentation functions which enhance or deplete high $p_{T}$ direct photon yields in heavy-ion collisions have also been proposed. Finally, distinguishing direct photons from $\pi^{0}$ decay photons producing an overlapping shower in the PHENIX calorimeter becomes increasingly difficult at $p_{T}>14 \mathrm{GeV} / c$.

However, RHIC's ability to provide collisions over a wide energy range may allow some means of separation. If the suppression at $200 \mathrm{GeV}$, as shown in Fig. 31, is indeed only an isospin effect, it should scale with $x_{T}=2 p_{T} / \sqrt{s}$. Therefore, in lower energy collisions it should manifest itself at lower $p_{T}$ where the experimental difficulties due to overlapping showers are absent. PHENIX measured a similar photon $R_{A A}$ in $62 \mathrm{GeV} \mathrm{Au+Au} \mathrm{collisions} \mathrm{using} \mathrm{NLO} \mathrm{pQCD}$ calculations for $p p$ (preliminary data from PHENIX on direct photon production in $p p$ at $62 \mathrm{GeV}$ will be available soon.) The results for the $10 \%$ most central collisions is shown in Fig. 32. While within errors it is consistent with unity, using measured data in the ratio may change this conclusion, as was the case at $200 \mathrm{GeV}$.

Although not an electromagnetic probe per se, the $\pi^{0} R_{A A}$ in Figs. 33 and 34

$\overline{20}$ This implies that while $p p$ data are the proper reference for the hadron $R_{A A}$, in the photon case the proper mixture of $\sigma_{p p}, \sigma_{p n}$, and $\sigma_{n n}$ must be used. For example, in minimum bias collisions $\sigma_{A A} / N_{\text {coll }}=\left(1 / A^{2}\right)\left(Z^{2} \sigma_{p p}+2 Z(A-Z) \sigma_{p n}+(A-Z)^{2} \sigma_{n n}\right)$. 


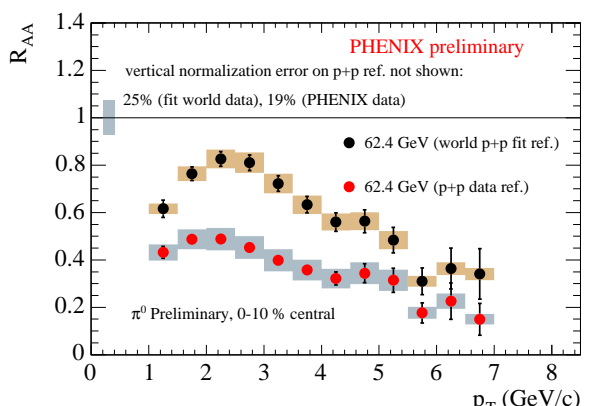

Fig. 33. Preliminary PHENIX $\pi^{0} R_{A A}$ in central $62 \mathrm{GeV} \mathrm{Au}+\mathrm{Au}$ collisions using a fit to the world average of previous $p p$ data (black circles) and a PHENIX $p p$ measurement (red circles).

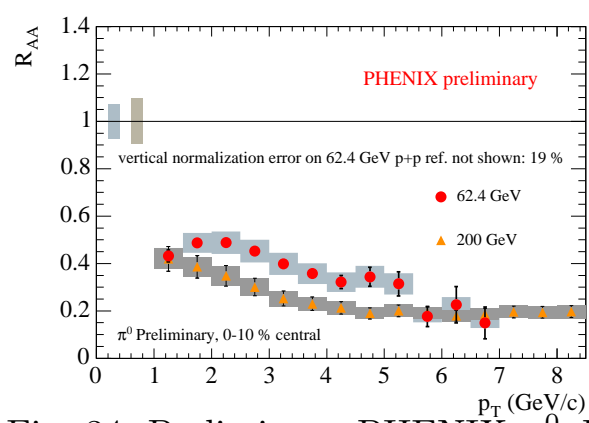

Fig. 34. Preliminary PHENIX $\pi^{0} R_{A A}$ in central $62 \mathrm{GeV}$ (red circles) and 200 $\mathrm{GeV}$ (orange triangles) $\mathrm{Au}+\mathrm{Au}$ collisions. Both ratios use PHENIX $p p$ measurements.

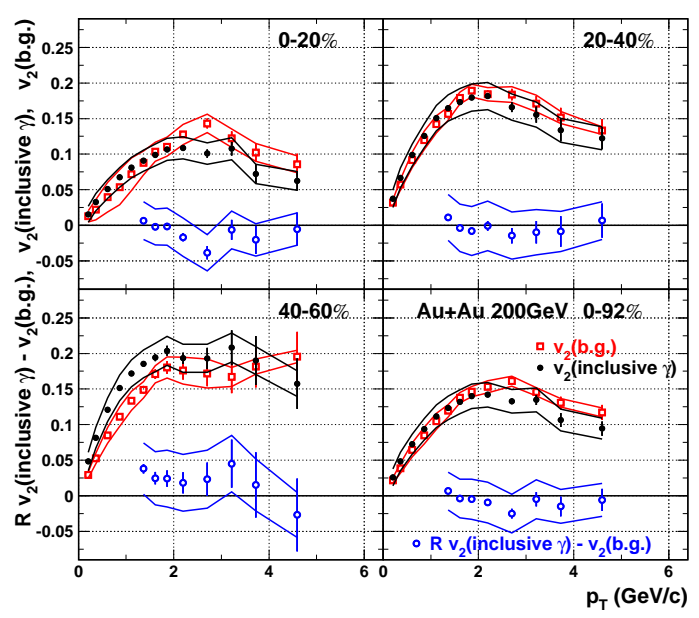

Fig. 35. The measured $v_{2}$ of inclusive photons ( $v_{2}^{\text {inc }} \gamma$, solid circles) and expected photon $v_{2}$ from hadronic decays $\left(v_{2}^{\mathrm{bg}}\right.$, open squares) [163]. The subtracted quantity, $R v_{2}^{\text {inc } \gamma}-v_{2}^{\mathrm{bg}}$ is plotted at the bottom of each panel (open circles). Here $R=\left(N_{\text {dir } \gamma}+N_{\mathrm{bg}}\right) / N_{\mathrm{bg}}$ corresponds to the product $\left.v_{2}^{\text {dir }} \gamma(R-1)\right)$. Four centralities are shown, including the minimum bias result.

illustrates the importance of measuring the reference in the same experiment with similar systematic errors. Fig. 33 compares the previous and the current preliminary $\pi^{0}$ suppression in $62 \mathrm{GeV} \mathrm{Au+Au}$ collisions. The earlier result used a fit to the ISR $p p$ data (upper, black circles) whereas the new result uses the $p p$ cross sections now measured in the same experiment (PHENIX). The two results in Fig. 33 would clearly lead to very different physics conclusions. Fig. 34 shows the new $62 \mathrm{GeV}$ result compared to the $R_{A A}$ obtained in 200 GeV collisions. 


\subsection{Photon Azimuthal Asymmetries (Elliptic Flow)}

Fig. 1 showed that the overall azimuthally-integrated high- $p_{T}$ photon yields scale with $N_{\text {coll }}$ and are well described by pQCD within current experimental and theoretical uncertainties. However, this global agreement may mask more subtle effects. It is even possible that the agreement is only accidental, due to cancellations of processes that enhance and quench the photon yield. An important step toward clarifying the level of agreement is the study of azimuthal asymmetries in the photon distributions, specifically their elliptic flow, $v_{2}$. If photons from the initial hard scattering do not interact with the medium, their $v_{2}$ is expected to be zero. However, initial hard scattering is not the only source of photons in $A A$ collisions. They may also originate from jet-thermal parton interactions or from Brehmsstrahlung off a quark. These photons are expected to exhibit a negative $v_{2}[161,162]$ (since more material is traversed out-of-plane, the major axis in coordinate space) with a strong $p_{T}$-dependence. However, photons from thermal radiation should reflect the dynamical evolution of the hot and dense matter, thus carrying a positive $v_{2}$.

The first measurement of photon elliptic flow at RHIC is shown in Fig. 35 [163]. The measurement is quite delicate due to the large background from $\pi^{0}$ decay-photons that inherit the $v_{2}$ of the parent $\pi^{0}$. While the measured $v_{2}$ of inclusive photons is consistent with the $v_{2}$ of hadronic decay photons, i.e. a zero net direct photon flow, the errors are appreciable and the directto-inclusive photon ratio is very small at low $p_{T}$. The quality of the current data is insufficient to verify the predictions [161,162], including the sign of the net flow. Much higher statistics can help remedy the situation, at least at higher $p_{T}$. Although the net direct photon flow is predicted to decrease, the statistical errors will become smaller and, equally important, the directto-inclusive photon ratio will increase dramatically. But even at high $p_{T}$ the net flow will be a competition between processes with positive and negative $v_{2}$. New analysis techniques may be able to statistically disentangle isolated and non-isolated direct photons in heavy-ion collisions. Jet-photon conversions primarily produce isolated photons [161] with negative $v_{2}$. The magnitude of this flow depends strongly both on $p_{T}$ and the jet energy-loss mechanism in heavy-ion collisions. Therefore, a measurement of the isolated photon $v_{2}$ may provide an independent constraint on energy-loss models.

\subsection{Electron $R_{A A}$ and Flow}

The recent measurement of "non-photonic" single-electron spectra, associated with decays of open heavy-flavor hadrons, at RHIC lead to two unexpected and very important results: (i) the nuclear modification factor, $R_{A A}$, shows a 

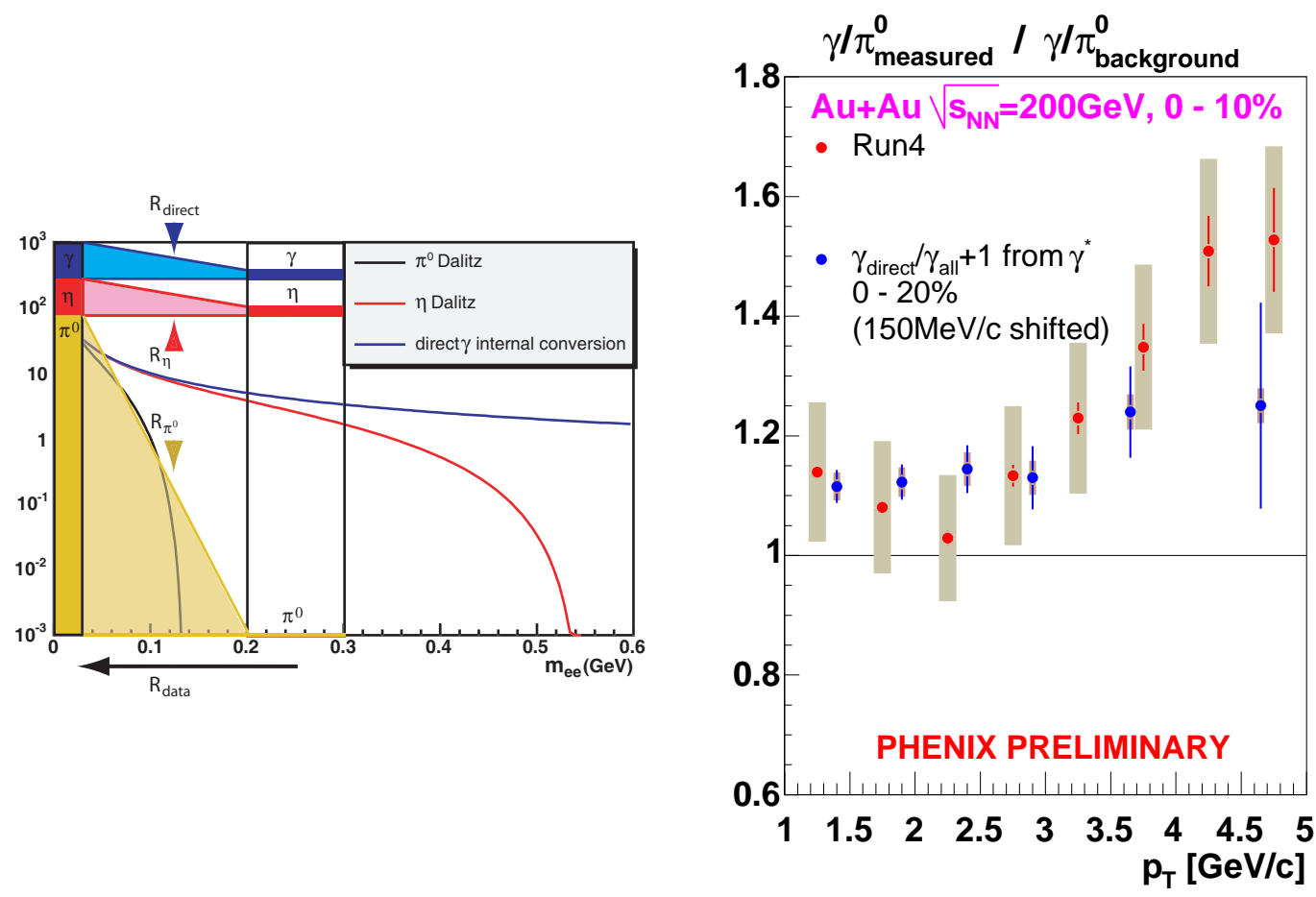

Fig. 36. Left: illustration of the direct photon measurement via low-mass dileptons [72]. Right: preliminary results on low- and intermediate- $p_{T}$ direct photons, the "photon excess ratio", with the traditional calorimeter method (red circles) and low mass electron pairs (blue circles).

strong suppression in central $\mathrm{Au}+\mathrm{Au}$ collisions $[164,165,166]$, comparable to the suppression observed for pions; (ii) the elliptic flow, $v_{2}$, is significant at low $p_{T}$, up to $10 \%$. Although such strong suppresion was predicted a decade ago [167], it was later argued that the suppression would be mild due to the "dead cone effect" for heavy quarks. Measurements from both STAR and PHENIX have, in fact, shown strong charm suppression and significant charm flow (early thermalization) in $200 \mathrm{GeV} \mathrm{Au+Au} \mathrm{collisions:} \mathrm{observations} \mathrm{not}$ simultaneously explained by current theories. These issues primarily concern charm and bottom physics and are discussed in great detail in the heavy flavor part of this Report. However, they may be significant for "classic" electromagnetic probes as well since heavy flavor suppression actually aids measurements of intermediate mass continuum dilepton radiation by reducing the combinatorial background from charm 21 in this regime, see Fig. 39. 


\subsection{Direct Thermal Photons via Low-Mass Dielectrons}

A very promising approach for measuring low- $p_{T}$ direct photons is to utilize low-mass electron pairs from "internal conversions", as was first applied in heavy-ion collisions by PHENIX [72]. The basic idea is that any process producing a real photon can also produce a very low mass virtual photon [169] which subsequently decays into an $e^{+} e^{-}$pair. This direct photon signal competes, of course, with dielectrons from $\pi^{0}, \eta$, etc Dalitz decays. The dielectron rates and mass distributions are described both for low-mass direct photons and Dalitz decays by the Kroll-Wada formula [169],

$$
\frac{1}{N} \frac{d N_{e e}}{d m_{e e}}=\frac{2 \alpha}{3 \pi} \sqrt{1-\frac{4 m_{e}^{2}}{m_{e e}^{2}}}\left(1+\frac{2 m_{e}^{2}}{m_{e e}^{2}}\right) \frac{1}{m_{e e}}\left|F\left(m_{e e}^{2}\right)\right|^{2}\left(1-\frac{m_{e e}^{2}}{M^{2}}\right)^{3},
$$

where the form factor, $F$, is unity for real photons. Note that the phase space for Dalitz decays is limited by the mass of the parent meson, $m_{e e}<M_{\pi^{0}, \eta, \omega}$, while for direct photons it is not, $m_{e e} \sim p_{T}$. Therefore, the measurement becomes relatively clean for $p_{T}>1 \mathrm{GeV} / c$, still in the low- $p_{T}$ realm where "traditional" calorimeter measurements have serious difficulties. The method is illustrated in the left panel of Fig. 36 while the resulting photon excess ratios are shown on the right. The systematic errors are much smaller for the new method 22 . The statistical errors become large for $p_{T}>4 \mathrm{GeV} / c$ although $\sim 15$ times more data have been analyzed for dielectrons (solid red circles) than for the calorimeter analysis (open blue circles). There is an important lesson here: even signals such as thermal photon radiation, typically not considered to be "starving for statistics", novel but promising new analyses techniques require large luminosity increases.

Before drawing any conclusions about the thermal nature of the apparent direct photon signal at low $p_{T}$ the baseline in $p p$ has to be established. This is shown on the left panel of Fig. 37 (final results from PHENIX): the signal is in good agreement with NLO pQCD predictions. In contrast, in $\mathrm{Au}+\mathrm{Au}$ (right panel) there is clearly an additional source at low $p_{T}$.

The conversion approach is also appealing from the theoretical point of view since the framework outlined in Sect. 2.1 accommodates a comprehensive treatment of real and virtual photons, as represented by Eqs. (1) and (2). However, additional theoretical work is needed for a more complete assessment of possible very-low mass dielectron sources [21].

$\overline{21}$ This background is irreducible for the PHENIX HBD.

${ }^{22}$ The errors are smaller, at least for the direct photon excess ratio. However, the absolute normalization and the direct photon cross sections are difficult to assess and therefore have been inferred using the calorimeter. 


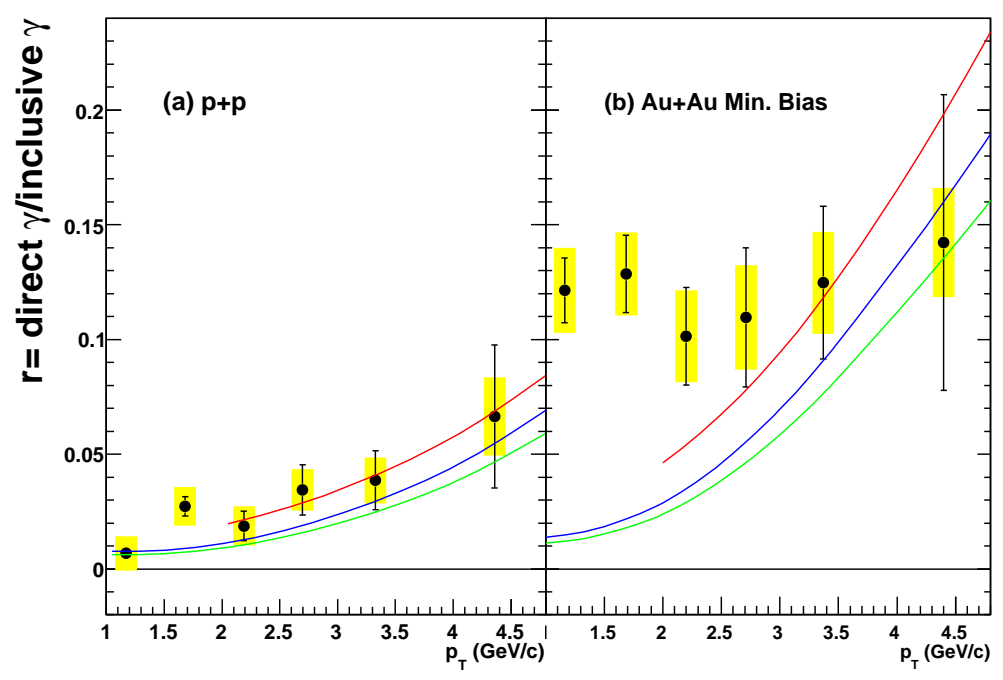

Fig. 37. The fraction of the direct photon component as a function of $p_{T}$ in $200 \mathrm{GeV}$ $p p$ (left) and $\mathrm{Au}+\mathrm{Au}$ (right) collisions. The error bars and the error band are the statistical and systematic uncertainties, respectively. The curves are from NLO pQCD calculations.

\section{THE FUTURE}

\subsection{Detector upgrades}

The original designs of both PHENIX and STAR anticipated later upgrades to enhance their physics capabilities. In fact "upgrading" as a response to spectacular rises in luminosity and physics insights from data already taken was and remains an almost continuous process from the very start of operations. Few can be tied uniquely to RHIC-II with the exception of upgrades facilitating the handling of high luminosities and data rates. Nonetheless, several major projects should be discussed here because of their magnitude and their impact on the future capabilities of RHIC-II, see Table 1. These projects are in different stages of development. Some are in the early R\&D phase while others are ready to be installed soon. Here we concentrate on upgrades that are particularly important for electromagnetic probes. The STAR Time-ofFlight (TOF) detector and Heavy Flavor Tracker (HFT) improve the electron identification and the displaced vertex measurement by rejection of electrons from charm, bottom and Dalitz decays as well as conversion electrons. The PHENIX Hadron Blind Detector (HBD) will reject Dalitz pairs for light vector meson measurements. The Silicon Vertex Detector (SVTX) will measure displaced vertices to identify electrons from open charm. The Nose-Cone Calorimeter (NCC) will measure photons and $\pi^{0}$ 's at forward rapidities. A new high-resolution sampling calorimeter is proposed for STAR to measure 
direct photon correlations. In the following, we will discuss these systems in more detail.

\subsubsection{STAR Time-of-Flight and Heavy Flavor Tracker}

STAR electron identification is made possible by a combination of energy loss, $d E / d x$, by charged particles due to ionization of the time-projection chamber (TPC) gas and a velocity measurement with the TOF system [170]. The relativistic rise of the electron $d E / d x$ separates electrons from hadrons except at the crossovers with pions at $\sim 0.2 \mathrm{GeV} / c$, kaons at $\sim 0.6 \mathrm{GeV} / c$, protons at $\sim 1.1 \mathrm{GeV} / c$ and deuterons at $\sim 1.5 \mathrm{GeV} / c$. A time-of-flight measurement, with the requirement that $|1-\beta|<0.03$, eliminates slow hadrons and cleans up the crossovers, resulting in clean electron identification [170].

In addition to direct measurements of open-charm hadrons via $K \pi$ decays, the STAR HFT [171] will be a powerful means of discriminating primordial electrons from background electrons in the measurement of electromagnetic probes.

As discussed earlier, direct photon and lepton production is rare and overwhelmed by photons and leptons from electromagnetic decays of hadrons and $\gamma$ conversions to electrons, $\gamma \rightarrow e^{+} e^{-}$. The $\gamma$ conversions, to a large extent, occur in the detector material. The HFT detector will reduce the background electrons and positrons from these $\gamma$ conversions. By requiring hits in the HFT, electrons from photon conversion outside the HFT, i.e., in the Silicon Strip Detector (the upgraded STAR Silicon Vertex Tracker) and the TPC inner field cage, are rejected. To estimate the signal-to-background ratio in the vectormeson measurements, STAR adopted a reasonably conservative approach and assume that the HFT can reject $\gamma$ conversions by a factor of 10 [171]. Although the configuration of HFT has evolved significantly, the simulation of background rejection is not expected to change. Another electron background arises from semileptonic decays of heavy quarks. If heavy-quark spectra are extrapolated from $p p$ collisions, the dominant dilepton source in central $\mathrm{Au}+\mathrm{Au}$ collisions at intermediate mass [68] is due to semileptonic $c \bar{c}$ decays. With the large charm yield at RHIC, the latter are comparable to the yield from $\gamma$ conversions and $\pi^{0}$ and $\eta$ Dalitz decays after HFT rejection. Detailed simulations show that the HFT is capable of rejecting $\sim 75 \%$ of $e^{+} e^{-}$pairs from $D^{0}$ decay while preserving $50 \%$ of the direct $e^{+} e^{-}$pairs.

The large reduction in electron background will enable STAR to observe the electromagnetic signal from low-mass vector mesons and radiation of intermediate-mass dileptons with a few hundred thousand central $\mathrm{Au}+\mathrm{Au}$ events. The rejection of $\pi^{0}$ and $\eta$ Dalitz decays by a factor of three (single track) can be achieved by measuring both electrons of the pair, made possible 


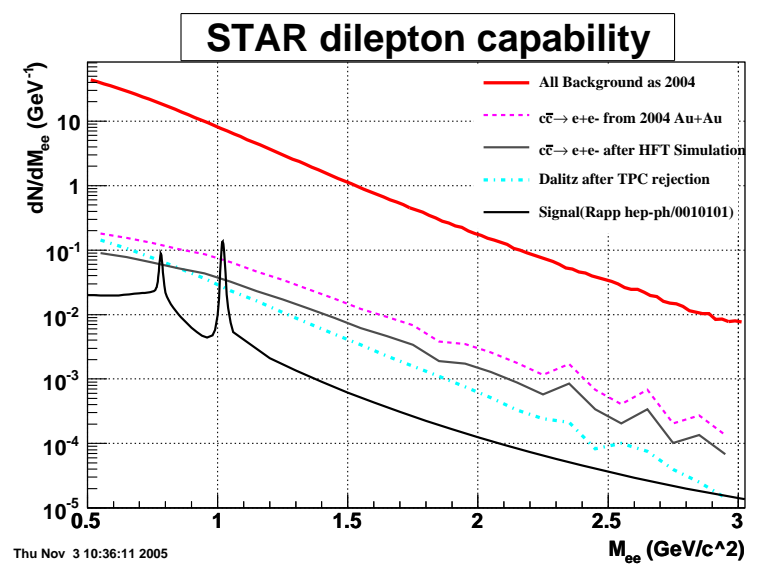

Fig. 38. Dielectron invariant mass distributions for central $200 \mathrm{GeV} \mathrm{Au+Au} \mathrm{colli-}$ sions. The solid black curve is the prediction for thermal emission [68] in the STAR acceptance. The red curve at the top is the total dielectron invariant-mass spectrum in the 2004 configuration assuming electron PID from full TOF coverage. The pink dashed line is the $e^{+} e^{-}$pair spectrum from semileptonic charm decays, the grey curve is the charm $e^{+} e^{-}$spectrum after HFT DCA $<80 \mu \mathrm{m}$, and the dot-dashed line are from $\pi^{0}$ and $\eta$ Dalitz decays after rejection by the TPC.

by the large acceptance of the STAR TPC. Future work on further rejection of such dilepton-background pairs with the inner tracker alone will be exploited. With the upgrades, STAR expects to detect $6 \mathrm{~K} \phi$ and $22 \mathrm{~K} \omega$ decays in 200 million recorded central $\mathrm{Au}+\mathrm{Au}$ collisions. These are to be compared with $\sim 10 \mathrm{~K}$ for the $\phi$ and $\sim 6 \mathrm{~K}$ for the $\omega$ in central $\mathrm{In}+\mathrm{In}$ collisions, presented by NA60 [156].

Fig. 38 summarizes the dielectron background and signal invariant-mass distributions. The signals of medium-modified vector mesons and thermal QGP radiation (black curve) are from calculations [68] folded over the STAR acceptance. The uppermost (red) curve is the total dielectron invariant-mass spectrum in the 2004 configuration, obtained from the single-inclusive electron spectrum measured in $200 \mathrm{GeV} \mathrm{Au+Au} \mathrm{collisions} \mathrm{assuming} \mathrm{electron} \mathrm{PID}$ from full TOF coverage. The pink dashed line is the $e^{+} e^{-}$spectrum from semileptonic charm decays derived from the non-photonic single-electron spectra measured in $\mathrm{Au}+\mathrm{Au}$ collisions. The gray curve is the charm $e^{+} e^{-}$distribution after applying the HFT distance of closest approach cut, DCA $<80 \mu \mathrm{m}$. The dot-dashed line is $\pi^{0}$ and $\eta$ Dalitz decays after TPC rejection. The net result is a signal-to-background ratio that, even in the continuum regime, is $\leq 0.1$, comparable to the central In+In NA60 measurements [7]. The standard way of dealing with the residual background is the mixed-event method, used by CERES and NA50/NA60 at the SPS and will be used in PHENIX and STAR.

With the proposed Time-of-Flight, Heavy-Flavor Tracker and Data Acquisition System (DAQ1000) upgrades, STAR will be able to take data at a rate 


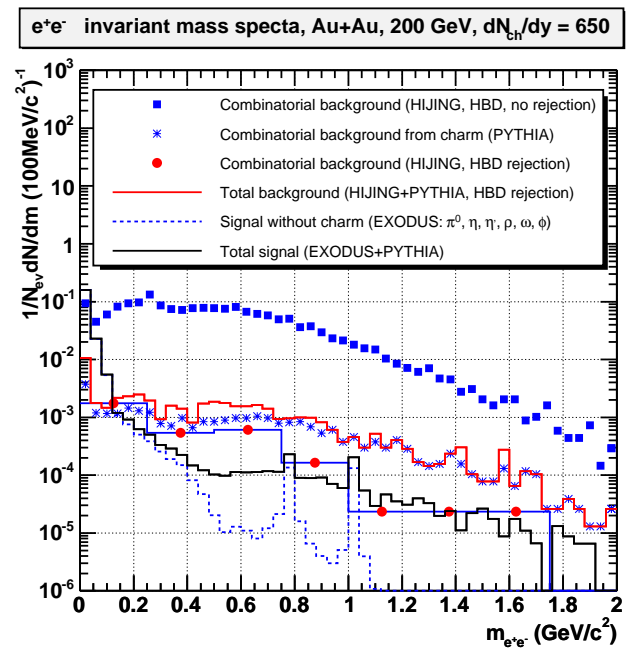

Fig. 39. Combinatorial background for low-mass electron pairs compared to the total signal from vector mesons and charm with and without the HBD. Blue squares: total $e^{+} e^{-}$combinatorial (HIJING), no rejection from the HBD. Red circles: combinatorial background after rejection with HBD. Blue stars: combinatorial background from charm alone (PYTHIA), not rejected by the HBD, measured separately by the Silicon Vertex Detector (SVTX). Solid red line: total combinatorial background after HBD rejection (HIJING+PYTHIA). Dashed blue line: pure signal from direct vector-meson and Dalitz decays after freezeout "cocktail" without the charm contribution (EXODUS). Solid black line: total signal from vector mesons, Dalitz decays and charm (EXODUS+PYTHIA).

of $1000 \mathrm{~Hz}$ with very little dead time. At the same time, the collision vertex has to be limited to $\sim \pm 5 \mathrm{~cm}$ due to the acceptance of the HFT [171]. The current data-taking rate is $50 \mathrm{~Hz}$ with a collision diamond of $\sim \pm 50 \mathrm{~cm}$. Without a machine upgrade, the average luminosity delivered to STAR is $8 \times 10^{26}$ $\mathrm{cm}^{-2} \mathrm{~s}^{-1}$ or $6 \mathrm{kHz} \mathrm{Au}+\mathrm{Au}$ minbias nucleus collision rate. Taking into account the factor of $3-5$ loss from the vertex constraint and centrality binning (e.g. 10\% most central), central-triggered $\mathrm{Au}+\mathrm{Au}$ events can be recorded at a rate of about $200 \mathrm{~Hz}$ with all the available luminosity, significantly below the DAQ1000 capability. A factor of 10 luminosity upgrade will enhance both the collider and detector. In peripheral collisions, the statistics will be lower due to lower event multiplicities. However, triggering will be more effective even for low- $p_{T}$ lepton pairs. Since the luminosity decreases quadratically with decreasing beam energy, an efficient energy scan at RHIC requires a luminosity upgrade, as discussed in more detail in Sect. 4.3.

\subsubsection{PHENIX Hadron Blind Detector and Silicon Vertex Detector}

As discussed earlier, the measurement of the low-mass electron pair continuum to infer the in-medium modifications of the light vector-meson $(\rho, \omega$, and $\phi)$ spectral functions is crucial for understanding the fate of hadron masses 
and the approach to chiral symmetry restoration in the hot, dense matter created in heavy-ion collisions. However, the $e^{+} e^{-}$signal is overwhelmed by the combinatorics from $\pi^{0}$ Dalitz decays and photon conversions. The combinatorial background from open-charm decays is also significant, see Fig. 39. Fortunately, the typical opening angle of the $e^{+} e^{-}$pair is very small, both for $\pi^{0}$ Dalitz decays and photon conversions. Thus unless they are swept apart by a magnetic field, a proximity cut on electron pairs is an effective veto on Dalitz and conversion electrons. The PHENIX magnet has an inner and an outer coil which are typically powered by currents in the same direction for maximum field to optimize tracking to the highest possible $p_{T}$. However, the current in the inner coil can be reversed, making the magnetic field vanishingly small around the collision region, allowing the installation of a Hadron Blind Detector (HBD) to eliminate the combinatorial background from Dalitz decays and conversions. This detector, described in Ref. [172], is a windowless Cherenkov detector operated with pure $\mathrm{CF}_{4}$ in proximity focus configuration with a CsI photocathode and a triple GEM detector with pad readout. It is a crucial upgrade for the exploration of the properties of the new matter created. As illustrated in Fig. 39 it will reduce the combinatorial background and increase the signal/background ratio from $\sim 10^{-3}$ to $\sim 10^{-1}$. The latter is again very comparable to NA60 and the expected performance of STAR after upgrades, described in the previous section.

The remaining background from open charm will be separately measured in PHENIX by a Silicon Vertex Detector (SVTX), which will measure the heavyflavor displaced vertex with a $40 \mu \mathrm{m}$ resolution of the distance of closest approach. The SVTX resolution is driven by the $c \tau$ of $123 \mu \mathrm{m}$ and $462 \mu \mathrm{m}$ for $D^{0}$ and $B^{0}$ decays, respectively. The SVTX will have a central barrel and two endcap detectors, covering both central and forward rapidities and providing inner tracking with full azimuthal coverage and up to $|\eta|<2.4$. This, in particular, will enable the measurement of correlated $e \mu$ invariant-mass spectra and thus provide a standalone determination of the correlated open heavy flavor component of the dilepton spectra.

\subsubsection{The PHENIX Nose-Cone Calorimeter}

PHENIX can measure photons, $\pi^{0}$ 's and $\eta$ 's well at midrapidity but the rapidity coverage of the electromagnetic calorimeter in the central arm is limited to $|y|<0.35$, making full jet reconstruction very difficult. Also, several large $y$ measurements in $\mathrm{d}+\mathrm{Au}$ collisions suggest that low- $x$ gluons might be saturated so that the $\mathrm{CGC}$ describes the results, including hadron suppression at large rapidity relative to no suppression at $y=0$. If the large $y$ suppression is indeed a consequence of initial-state gluon saturation, photons should also be suppressed, see Sec. 2.8. Such a study is only feasible with a calorimeter at large rapidities. The limited acceptance of the central arm also makes crucial 
$\gamma$-jet measurements very difficult.

PHENIX thus proposed to replace the current copper magnet nosecones by a calorimeter covering $1<|\eta|<3$. This Nose-Cone Calorimeter (NCC) is a silicon-tungsten sandwich sampling calorimeter, longitudinally segmented in three sections (two electromagnetic and one hadronic) and read out by $1.5 \times 1.5 \mathrm{~cm}^{2} \mathrm{Si}$ pads. In addition, two layers of $468 \mu \mathrm{m}$ pitch strip-pixels are added to achieve separation of direct photons from high-energy $\pi^{0}$ 's by shower-shape reconstruction. The total depth is $\sim 35 X_{0}$ radiation and $1.3 L_{\text {abs }}$ nuclear absorption lengths. The expected resolution for electromagnetic showers is $23 \% / \sqrt{E}$. The longitudinal segmentation allows to distinguish between electromagnetic and hadronic showers. The coverage is sufficient to reconstruct the entire jet energy.

Therefore, jet physics and energy-loss studies using both photon-tagged jets and leading $\pi^{0}$ 's will be possible far away from central rapidity with the NCC. It will also be very useful for studying $P$-state quarkonia via $\chi_{c} \rightarrow \gamma J / \psi$ and possibly $\chi_{b} \rightarrow \gamma \Upsilon$ radiative decays together with the muon spectrometer, giving information on the different quarkonium dissociation temperatures and feed down contributions to the $J / \psi$ and $\Upsilon$ yields [173,174]. The NCC is also an important part of the spin program by measuring the polarized gluon structure function at low $x$.

\subsection{New Measurements}

\subsubsection{Direct Photon Correlations (HBT)}

The HBT correlation of photons from $\pi^{0}$ or $\eta$ decays is exceedingly small because of the "large" distances, $>10^{7} \mathrm{fm}$, at which the decay occurs. Typical correlations from $\pi^{0}$ decay photons are $\mu \mathrm{eV} / c$ in relative photon fourmomentum. Therefore, any measurable HBT correlation will come from photons directly emitted from the collision.

Photon HBT correlations encode space-time information about the system emitting the photons. Many calculations, as well as "simple" quantum mechanics, indicate that by studying HBT in different $p_{T}$ regions, emitting systems of different temperatures and thus at different stages of evolution can be identified [175]. In addition, interferometry can also be used to measure the yield of direct photons [18].

While the effect of the $\pi^{0}$ decay background is to dilute the HBT signal, the decay in itself does not generate a fake correlation. However, the residual correlation of decay photons due to HBT $\pi^{0}$ correlations could cause a fake HBT correlation. When the measured HBT parameters determined from charged 
pions are added into a Monte Carlo calculation of the residual correlation, the change is negligible compared to reasonable estimates of the direct photon correlation assuming that the charged and neutral pion HBT parameters are the same [176].

The dilution effect, on the other hand, is a serious issue. Reasonable estimates of the visible HBT correlation indicate that $\lambda$, the effective amplitude of the HBT correlation, is only a few parts per thousand. Consequently, great care must be taken in designing experiments to measure the direct photon HBT. Corresponding work on this problem is briefly summarized in the remainder of this section, based on a more complete, technical paper [176].

STAR studies showed that the best way to measure the HBT correlation is to use one photon that converted in a thin $\left(10 \% X_{0}\right)$ converter placed inside the inner-field cage of the TPC and another (real) photon detected in the barrel calorimeter. It is thus possible to be sensitive to photons with nearly identical directions. However, in order to make useful measurements of the HBT parameters, the energy resolution of the electromagnetic calorimeter must be substantially improved over the resolution of the present calorimeter, $\delta E / E=15 \% / \sqrt{E}$. Pertinent studies show that $\delta E / E=3 \% / \sqrt{E}$ is certainly sufficient and some preliminary Monte Carlo studies indicate that even $5 \% / \sqrt{E}$ may be adequate albeit near the limit of usefulness. Work is in progress to determine the precise requirements, along with studies of the "Shashlyk" design, a relatively cheap calorimeter where alternating plates of radiator and scintillator are read out by an array of wavelength-shifting fibers which run parallel to the long axis of the calorimeter and penetrate the radiators and the scintillators. The technology is well established: a Shashlyk calorimeter used in E-865 at the AGS achieved 8\%/ $\sqrt{E}$ resolution, and ten years ago $75 \%$ of the PHENIX calorimeter was built with a similar design and shows similar performance. Subsequent studies done for the KOPIO experiment have demonstrated that a $3 \% / \sqrt{E}$ resolution is achievable. The designers of this calorimeter are now engaged in a design study of a Shashlyk calorimeter for STAR.

In subsequent feasibility studies, a model of direct photon production has been constructed which agrees with both existing measurements at $p_{T} \geq 2.0 \mathrm{GeV} / c$ and a recent calculation below that value [28]. The model assumes Bjorken (boost invariant) expansion, and thermal spectra from three regions of temperature at different times in each rapidity slice. The $\pi^{0}$ spectrum is taken from PHENIX data. The rapidity distribution is known from the PHOBOS measurement, however, in the STAR range $(\eta= \pm 1.0)$ it is effectively constant. An analysis procedure for the calorimeter has been devised which takes into account both the effects of isolation cuts and of unavoidable unrecognized overlaps of background photons. 


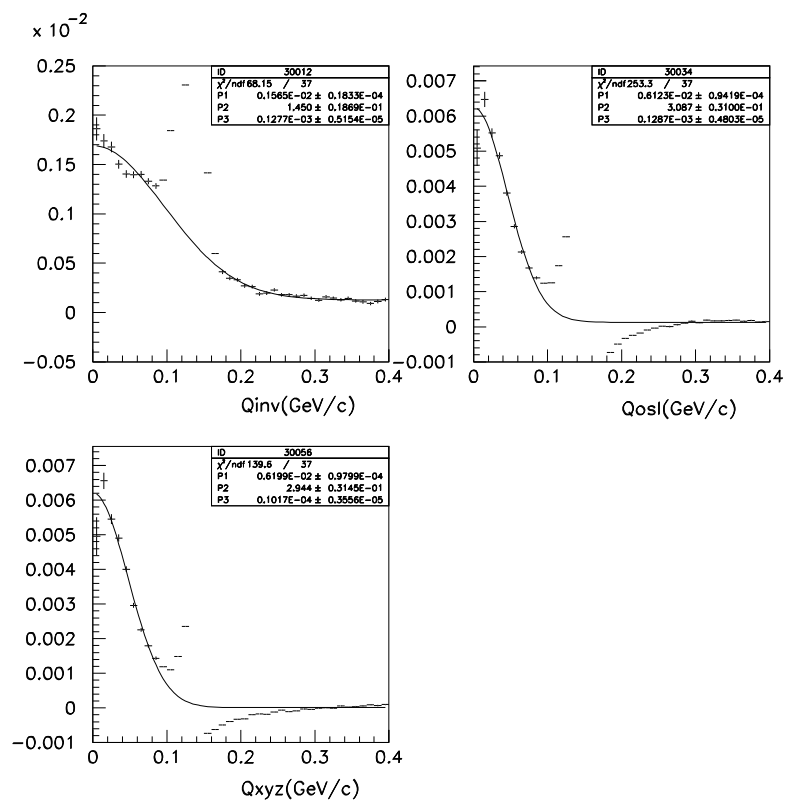

Fig. 40. Simulated correlation functions using 40 million events with full background, as described in the text. Top left: $Q_{\text {inv }}$, top right: $Q_{\text {osl }}$, bottom left: $Q_{x y z}$.

STAR can measure the direct photon yields and HBT parameters for $p_{T} \leq$ $600 \mathrm{MeV} / c$ with about 40 million events. Fig. 40 shows the simulated results for three one-dimensional correlation functions: $Q_{\text {inv }}$ is the usual invariant four-momentum difference; $Q_{\text {osl }}$ is the four-momentum difference in the usual out-side-long Bertsch-Pratt system [177]; and $Q_{x y z}$ is the four-momentum difference in the $x, y$, and $z$ Cartesian system. The figure shows that, despite the very low $\lambda$, quite good signals can be obtained. The individual out, side, and long radii are functions of the photon $k_{T}$, described in detail in a forthcoming paper [176]. The number of events necessary to observe direct photon correlations for $p_{T}$ between 1.0 and $2.0 \mathrm{GeV} / c$ is being studied, but it would clearly involve much larger data samples. An interesting possibility is to use the high $E_{T}$ trigger to access the higher $p_{T}$ region since it measures the system size at a different time [175].

\subsubsection{Spectral Distributions of the $a_{1}$ Meson}

An experimental measurement of the iso-vector-axial vector $\left(a_{1}\right)$ spectral function is desirable because of its important role in the search for chiral symmetry restoration. It is related to measurements of thermal low-mass dileptons, dominated by the $\rho$ (iso-vector-vector correlator), the chiral partner of the $a_{1}$, see Sec. 2.7. It has been suggested in [129] to make such a measurement via the $\pi^{ \pm} \gamma$ invariant-mass spectra. Calculations of photons from $a_{1}$ decays have concentrated on the inclusive photon yield. It is very difficult to isolate the $a_{1}$ contribution and determine the spectral shape of the $a_{1}$ from inclusive measurements alone. This is the main motivation for measuring the associ- 


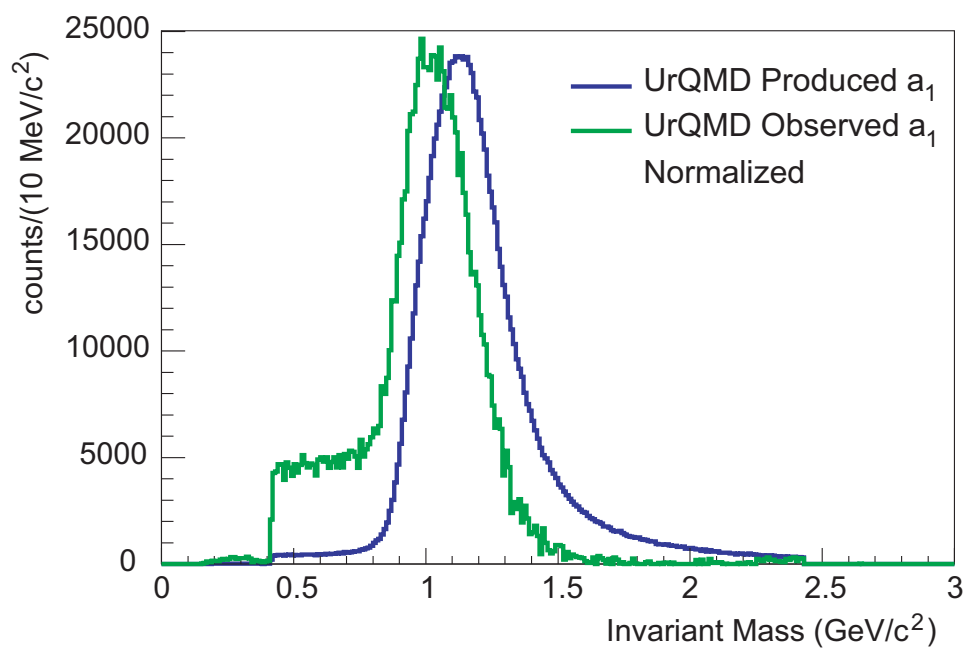

Fig. 41. The spectral shape of the initially-produced $a_{1} \rightarrow \gamma \pi$ (blue line) compared to the spectral shape of the observed $a_{1} \rightarrow \gamma \pi$ which have passed through the medium without being absorbed (green line), normalized to the number of produced $a_{1}$.

ated charged pion even though they experience strong final-state interactions, including absorption. Therefore, the in-medium spectral information will primarily pertain to the more dilute stages of a heavy-ion reaction. However, the $a_{1}$ may undergo significant modifications even at moderate temperatures and densities [178]. Such effects have possibly been seen in the $\rho \rightarrow \pi^{+} \pi^{-}$[179] decay, which is "penalized" by two pion absorption factors. Important information about chiral symmetry restoration may thus be obtained together with the dilepton measurements of the $\rho$ meson.

We report on studies [180] of the $a_{1} \rightarrow \gamma \pi$ channel using transport model simulations, i.e., minimum bias $\mathrm{Au}+\mathrm{Au}$ UrQMD events [181] at $\sqrt{s_{N N}}=200$ $\mathrm{GeV}$. In UrQMD, the $a_{1}$ was introduced with $B\left(a_{1} \rightarrow \gamma \pi\right)=0.1, B\left(a_{1} \rightarrow\right.$ $\rho \pi)=0.9, M_{a_{1}}=1230 \mathrm{MeV}$, and $\Gamma_{a_{1}}^{\mathrm{tot}}=400 \mathrm{MeV}$. Only $5 \%$ of the produced $a_{1}$ 's are not absorbed in the medium. Of these unabsorbed $a_{1}$ 's, $80 \%$ decay via $\gamma \pi$ and $20 \%$ via $\rho \pi$. The spectral shape of $a_{1} \rightarrow \gamma \pi$ that are not absorbed (green line in Fig. 41) is significantly different from the spectral shape of the produced $a_{1}$ 's (blue line in Fig. 41). The peak is shifted toward lower masses by about $200 \mathrm{MeV}$. Thus, the extraction of medium effects seems feasible but requires a good understanding of final-state absorption effects.

At RHIC-II, the $a_{1} \rightarrow \gamma \pi$ decay can be measured using the STAR full coverage TOF. In STAR, the efficiency of measuring a conversion $\gamma$ is 5\% [182]. Therefore, $55 \mathrm{M}$ minimum bias $\mathrm{Au}+\mathrm{Au}$ collisions are necessary to measure a $3 \sigma$ signal (using HIJING to estimate the background). If a Shashlyk calorimeter measuring low-momentum $\gamma$ 's could be used in STAR, the $a_{1}$ efficiency could improve by a factor of 10 . 

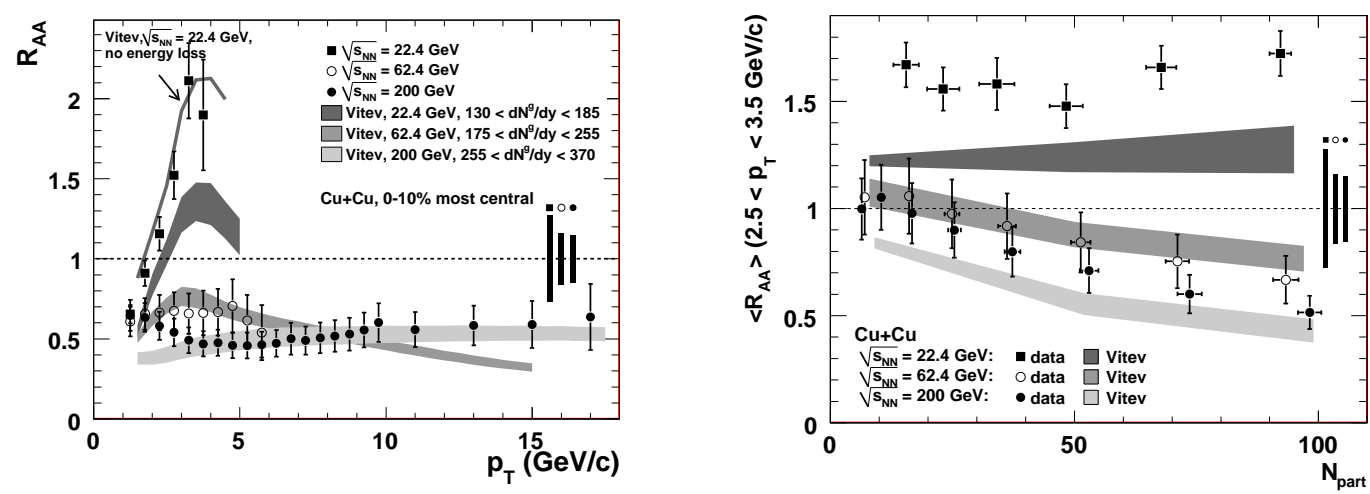

Fig. 42. Left: Measured $\pi^{0} R_{A A}$ as a function of $p_{\mathrm{T}}$ for the $0-10 \%$ most central $\mathrm{Cu}+\mathrm{Cu}$ collisions at $\sqrt{s_{N N}}=22.4,62.4,200 \mathrm{GeV}$ in comparison to a jet quenching calculation. The error bars in this figure represent the quadratic sum of the statistical uncertainties and the point-to-point uncorrelated and correlated systematic uncertainties. The boxes around unity indicate uncertainties related to $\left\langle N_{\text {coll }}\right\rangle$ and absolute normalization. The bands for the theory calculation correspond to the assumed range of the initial gluon density $\mathrm{d} N^{\mathrm{g}} / \mathrm{d} y$. The thin solid line is a calculation without parton energy-loss for central $\mathrm{Cu}+\mathrm{Cu}$ at $\sqrt{s_{N N}}=22.4 \mathrm{GeV}$. Right: The average $R_{A A}$ in the interval $2.5<p_{\mathrm{T}}<3.5 \mathrm{GeV} / c$ as a function of centrality for $\mathrm{Cu}+\mathrm{Cu}$ collisions at $\sqrt{s_{N N}}=22.4,62.4$, and $200 \mathrm{GeV}$. The shaded bands represent jet quenching calculations at three discrete centralities $\left(N_{\text {part }} \sim 10,50,100\right)$. The boxes around unity represent the normalization and $\left\langle N_{\text {coll }}\right\rangle$ uncertainties for a typical $N_{\text {coll }}$ uncertainty of $12 \%$.

\subsection{High Statistics and Energy/Species Scan}

The RHIC-II project calls for a ten-fold increase in the luminosity currently provided by RHIC, essential for full exploration of rare probes and very high- $p_{T}$ signals that are currently statistics-limited. Examples include detailed heavy quarkonium spectroscopy, more precise values and $p_{T}$-evolution of photon and hadron $R_{A A}$ at very high $p_{T}$, as well as photon flow and HBT at high $p_{T}$. Photon-tagged jets, particularly if measured relative to the reaction plane, would give further insight to energy loss mechanisms along with the complementary jet-dilepton correlations. At the current luminosity, it would take several years to collect sufficient statistics for these measurements with just one species and one energy setting: clearly inadequate for exploring some of the key questions about the sQGP in depth and in a timely fashion.

There are, however, additional physics opportunities. The above measurements investigate the properties of the sQGP above $T_{c}$, certainly a necessary task. But, as emphasized in the Introduction, while the existence of a new state of matter has been reasonably well established at RHIC, key features of the phase transition itself, including variations across the $T-\mu_{B}$ phase diagram, have not yet been mapped out. The only way to do this is by a detailed energy/species 
scan, only possible over a reasonable time scale with RHIC-II luminosities. The prospects are well illustrated by Fig. 42 which shows $\pi^{0} R_{A A}$ in $\mathrm{Cu}+\mathrm{Cu}$ collisions for $22.4 \mathrm{GeV}$ (close to the SPS range) to the highest RHIC energy, all measured by the same experiment (PHENIX). The transition from $R_{A A}>1$ to strong suppression occurs in this energy range. Clearly this doesn't exclude the possibility of some suppression already at SPS energies, but the (Cronin) enhancement is still dominant - and essentially constant - at all centralities, whereas at $62.4 \mathrm{GeV}$ the suppression increases with centrality.

At current RHIC luminosities, even with the accelerator running for $\sim 22-28$ weeks per year, the practical limit is at most two species or three energies per run period. The dominant part of the run is spent on actual collisions and data taking rather than changing settings. Since low $p_{T}$ observables (bulk observables, thermal photons and dileptons, and hadron ratios) explore the phase transition, long runs are not required to obtain reasonable statistics for a given energy/species setting. Indeed, with the luminosity upgrade, sufficient data could be collected in a few weeks at most settings, even after the luminosity loss with $\gamma^{-2}$ is accounted for.

The baryon chemical pontential, $\mu_{B}$, can be varied by changing $\sqrt{s_{N N}}$. RHIC was designed to vary $\sqrt{s_{N N}}$ and has successfully done so in several runs. The RHIC experiments have collected heavy-ion data at $\sqrt{s_{N N}}=22,62,130$ and $200 \mathrm{GeV}$, covering several points in the $\sqrt{s_{N N}}$ interval between the SPS and the maximum RHIC energy. Recent studies have shown that RHIC could run collisions as low as sqrts $_{N N}=5 \mathrm{GeV}$ - the AGS range - to compare to previous AGS and SPS experiments, as well as fill in gaps in earlier measurements. While this may at first appear to be a repetition of earlier work, we want to re-emphasize the value of doing measurements over the entire energy range with the very same apparatus. Since most systematic errors are the same, the evolution of physics quantities characterizing the transition can be traced to higher precision and with more reliable systematics.

Lower energies, however, come at the price of an approximately quadratic luminosity decrease with $\sqrt{s_{N N}}$. Therefore, as indicated at the end of Sec. 4.1.1, the RHIC-II luminosity upgrade is critical for a meaningful energy-scan program for low-mass dileptons. For example, the RHIC-II luminosity at $\sqrt{s_{N N}}=$ $30 \mathrm{GeV}$ will be the same as the current maximum-energy luminosity. Without upgrade, a factor of 40 or more luminosity loss from $\sqrt{s_{N N}}=200 \mathrm{GeV}$ to $\sqrt{s_{N N}}=30 \mathrm{GeV}$ would make an energy scan program essentially useless for dileptons. Based on discussions in Secs. 2.6, 2.7.2 and 3.1, a minimal $\sim 20 \%$ accuracy with a $\sim 2 \%$ mass resolution is needed to develop sufficient discriminating power between even rather distinct in-medium modification scenarios. Obviously, future theoretical refinements will further raise these requirements. Direct photon flow measurements up to $p_{T} \sim 8-9 \mathrm{GeV}$ are needed to investigate contributions from parton fragmentation, jet-thermal interactions and 
Bremsstrahlung off quarks. Current luminosities limit the measurements to $\sim 5-6 \mathrm{GeV}$, see Sec. 3.5. A tenfold luminosity increase would extend the $p_{T}$-range by the necessary $2-3 \mathrm{GeV}$. Interferometry of pre-hadronic photons (emitted before the hadronic phase), $p_{T}>2 \mathrm{GeV}$ [175], with the precision deduced in Sec. 4.2.1 requires about $7 \times 10^{8}$ events which would, with current luminosities, allow measurements with only one system and/or energy per year. Also, many of the intermediate invariant mass and transverse momentum, $M, p_{T} \sim 2 \mathrm{GeV}$, signals, where sQGP radiation dominates (see Secs. 2.4.1 and 2.5.3), can be triggered, making high luminosity extremely valuable in this context as well. Photon-based temperature measurements, described in Sec. 2.4.2, depend on detailed energy and species scans, including sufficient $p p$ reference data at the same energies as $A A$ collisions instead of relying on earlier results from other accelerators 23 . Once again, the benefits of measuring both spectra in the same detector are obvious: in many cases the systematic errors decrease by $40-50 \%$.

Colliding different ion species allows variation of the collision geometry at fixed participant number, $N_{\text {part }}$. So far at RHIC several bulk observables have been found to scale with $N_{\text {part }}$ irrespective of collision geometry. Recently it has been found that even more subtle quantities such as the azimuthallyintegrated nuclear modification factor, $R_{A A}$, is similar in central $\mathrm{Cu}+\mathrm{Cu}$ collisions and mid-peripheral $\mathrm{Au}+\mathrm{Au}$ collisions with the same $N_{\text {part }}$. However, once azimuthal distributions are studied, significant differences emerge since the two collision geometries are not alike (spherical $\mathrm{Cu}+\mathrm{Cu}$ vs ellipsoidal $\mathrm{Au}+\mathrm{Au}$ ) and these different geometries are crucial for understanding processes such as inmedium energy loss. Also, for sufficiently light ions, thermalization, apparent in the hydrodynamic behavior of the $\mathrm{Au}+\mathrm{Au}$ and even $\mathrm{Cu}+\mathrm{Cu}$ data, can no longer be achieved. Pinpointing the thermalization boundary could be crucial for determining the behavior of probes near the phase boundary and the conditions needed for the transition to occur.

We reiterate that higher luminosities can be used to increase the statistics of a particular data set, gaining access to rare probes, and also to increase the number of ion species (symmetric or asymmetric collision systems) and/or the energy settings by subdividing the RHIC runs since many important signals do not require very high statistics. The accelerator is the most flexible ever built and can run multiple system/energy combinations per year if the physics justifies it. In fact, RHIC produced the very first collisions at $\sqrt{s_{N N}}=9 \mathrm{GeV}$ in a 12 hour test in June 2007. 


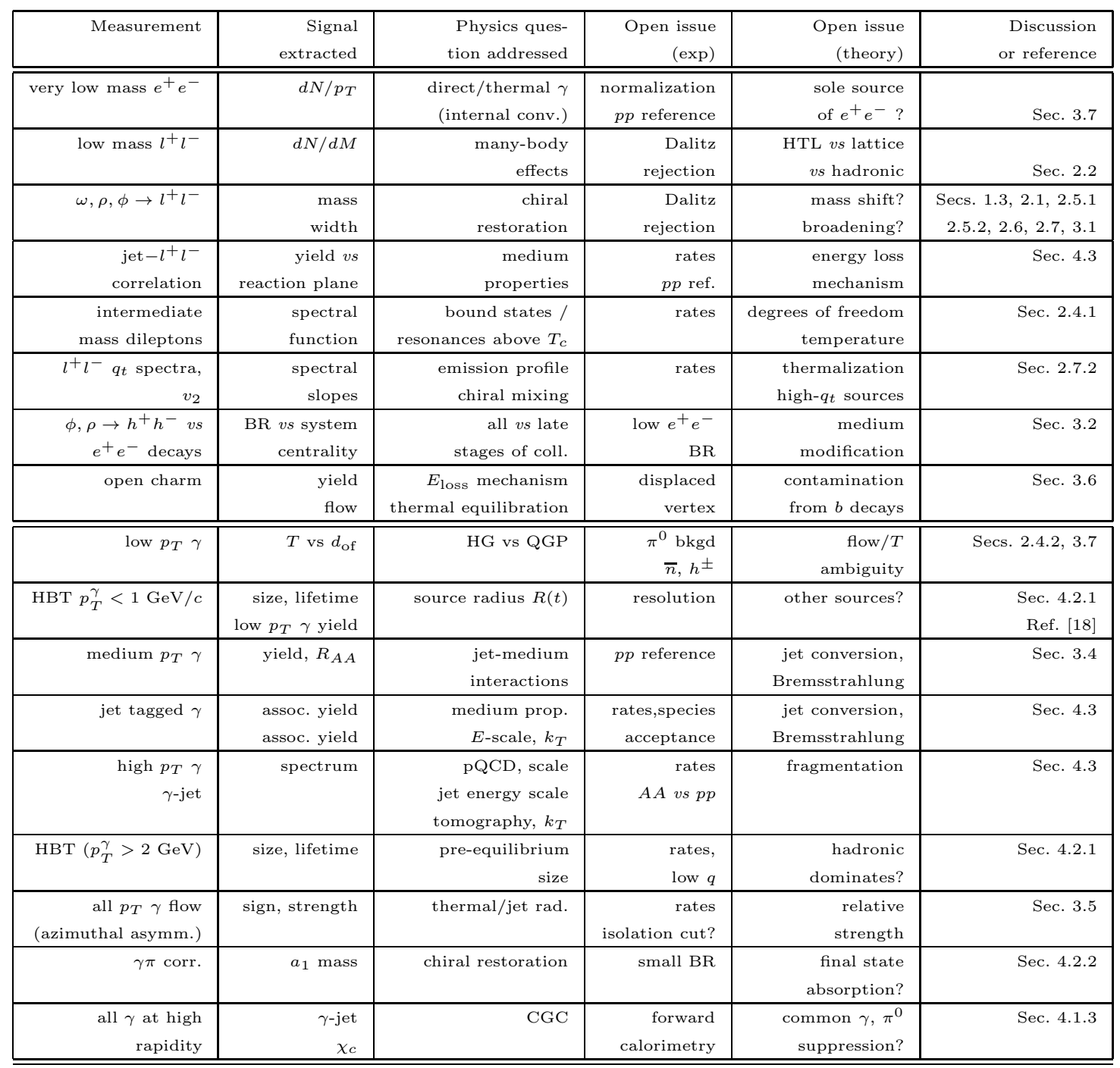

Table 2

Bottom-up view of electromagnetic probes in heavy-ion collisions: from observables to the pertinent theoretical issues addrssing the underlying physics questions.

\subsection{From Observables to Pertinent Theoretical Issues}

In Table 2 we summarize how electromagnetic probes can influence the theoretical understanding of relativistic heavy-ion collisions. In the first column we list specific measurements, in the second the signal extracted either directly or with minimal model assumptions, and the physics question addressed in the third column. The fourth column describes the most important experimental issues and the fifth indicates open or ambiguous theory questions that require significant further investigation. Finally, the sixth column points either to a Section of this review or to a reference where the issues are discussed in more detail.

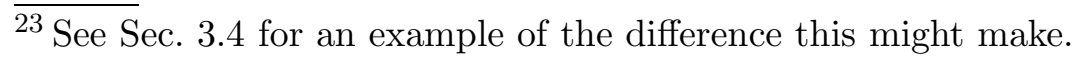


While this table has a level of subjectivity, it reflects our current thinking on the most interesting issues related to EM probes at RHIC-II. We do not list topics that are primarily or exclusively the domain of the LHC or the future Electron-Ion Collider (EIC).

\section{SUMMARY AND CONCLUDING REMARKS}

We now summarize our opinion of the most promising developments in connection with EM probes at future RHIC experiments.

Low-mass (axial-) vector-meson spectroscopy close to the chiral transition Precision dilepton data will provide detailed information on medium modifications of $\rho, \omega$ and $\phi$ mesons, and thus illuminate the question of hadronic mass (de-)generation. "Quality control" of theoretical models via independent constraints from symmetries, QCD sum rules and phenomenology is essential to limit the scope of viable axial/vector $(A / V)$ spectral functions. An excitation function measurement will discriminate temperature and net-baryon density effects to systematically map out in-medium effects across a significant part of the QCD phase diagram. Information on chiral symmetry should be inferred from a novel measurement of the axial vector spectral function via $\pi^{ \pm} \gamma$ invariant-mass spectra as well as through a well-defined combination of effective chiral hadronic models, chiral sum rules and finite-temperature QCD lattice computations of chiral order parameters such as the pion decay constant and four-quark condensates.

The required theoretical tools are largely in place: chiral effective models for realistic axial-/vector spectral functions at finite temperature can be used to calculate the temperature dependence of order parameters (moments of " $V-$ $A$ " spectral functions). Unquenched lQCD evaluations of the latter should be pursued with high priority. A convincing deconvolution of the vector correlator from the measured spectra will require reliable space-time descriptions of $A A$ collisions, expected to emerge from progress in hydrodynamical simulations and complementary transport models.

Footprints of chiral restoration are furthermore expected at dilepton masses around the $a_{1}$ mass, $1-1.5 \mathrm{GeV}$, due to "chiral $V-A$ mixing". To detect a continuum enhancement of less than a factor of two requires accurate charm and background determinations providing a signal with no more than $20 \%$ total error, achievable with the planned vertex-detector upgrades together with sufficient statistics. In addition, dilepton elliptic flow and transverse momentum spectra will provide additional information on the space-time emission profile at different invariant masses. 
The highest temperatures of the matter formed at RHIC

We have identified three promising regimes where the QGP is expected to be the dominant source of electromagnetic radiation: intermediate-mass dileptons $(1.5 \leq M \leq 3 \mathrm{GeV})$ and photons and low-mass dileptons at intermediate transverse momentum, $\left(1 \leq q_{t} \leq 3 \mathrm{GeV}\right)$. These measurements will be supplemented by $\gamma \gamma$ correlation analyses which yield complementary information on the temperature and fireball size.

$Q G P$ resonances

The only direct way to experimentally determine the existence of hadronic bound states/resonances in the sQGP is to search for a resonant dilepton signal. Due to the nature of QGP emission, the greatest sensitivity to pertinent vector states is in the $M=2 \mathrm{GeV}$ mass region, coinciding with current expectations from lattice QCD and effective models. RHIC provides optimal conditions for this search since the initial temperatures are close to the anticipated bound state dissolution temperatures.

As is the case for signatures of chiral mixing at lower masses, an accurate determination of background and charm sources is mandatory to restrict the total experimental error to below the $\sim 20 \%$ level. If the resonance states exist at masses below $\sim 1.5 \mathrm{GeV}$, discriminating them from chiral-mixing effects will be more involved, increasing the demand for accuracy and systematic centrality and excitation-function studies.

\section{Detector and luminosity requirements}

To achieve the required background rejection and obtain high-precision charm measurements, PHENIX needs the Hadron Blind Detector and vertex detector, while STAR needs the Time-of-Flight detector, Heavy Flavor Tracker and Data Acquisition System. Initial dilepton measurements can be made after these new detectors are in place. However, an energy scan with statistics comparable to recent NA60 data requires significant luminosity upgrades.

A summary is provided in Table 2 .

Based on the arguments given in this review, we believe that after the future RHIC detector and luminosity upgrades for heavy-ion collisions in the $10 \leq \sqrt{s_{N N}} \leq 200 \mathrm{GeV}$ regime, electromagnetic probes will, in a combined experimental and theoretical effort, result in decisive and unique new perspectives of QCD matter at high (energy-) density. In particular, new insights into hadronic mass generation mechanisms related to chiral symmetry restoration, thermal radiation at unprecedented temperatures, microscopic properties of the sQGP and system size measurements during the early matter evolution will be gained. 


\section{Acknowledgments}

We thank our colleagues J. Casalderrey-Solana, H. van Hees, B. Müller, P. Petreczky, T. Renk, J. Ruppert, K. Schweda, R. Seto, R. Vogt, and C. Woody for valuable input and discussions. We are particularly indebted to D. d'Enterria for providing us with Sec. 2.4.2 on "Direct Photons and Current RHIC Data", to J. Jalilian-Marian for Sec. 2.8 on "Electromagnetic Signatures of the Color Glass Condensate", to J. Sandweiss and his collaborators for providing us with Sect. 4.2.1 on "Direct Photon Correlations" of this mansucript and to P. Fachini for Sect. 4.2 .2 (their write-ups have been edited by the three authors to fit style and scope of the manuscript). RR has been supported in part by a National Science Foundation CAREER Award under grant PHY-0449489. XZB has been supported in part by a DOE Early Career Award and the Presidential Early Career Award for Scientists and Engineers.

\section{References}

[1] I. Arsene et al. [BRAHMS Collaboration], Nucl. Phys. A 757, 1 (2005).

[2] B. B. Back et al. [PHOBOS Collaboration], Nucl. Phys. A 757, 28 (2005).

[3] J. Adams et al. [STAR Collaboration], Nucl. Phys. A 757, 102 (2005).

[4] K. Adcox et al. [PHENIX Collaboration], Nucl. Phys. A 757, 184 (2005).

[5] Proceedings of the 18th International Conference on Ultra-Relativistic NucleusNucleus Collisions, Nucl. Phys. A 774 (2006), edited by T. Csorgo, G. David, P. Levai, and G. Papp.

[6] S. Damjanovic et al. [NA60 Collaboration], Nucl. Phys. A 774, 715 (2006).

[7] R. Arnaldi et al. [NA60 Collaboration], Phys. Rev. Lett. 96, 162302 (2006).

[8] S. S. Adler et al. [PHENIX Collaboration], Phys. Rev. Lett 94, 232301 (2005).

[9] L.E. Gordon and W. Vogelsang, Phys. Rev. D 48, 3136 (1993); Phys. Rev. D 50, 1901 (1994);

P. Aurenche et al., Phys. Lett. B 140, 87 (1984); Nucl. Phys. B 297, 661 (1988).

[10] G. Agakichiev et al. [CERES Collaboration], Eur. Phys. J. C 41, 475 (2005).

[11] G. E. Brown and M. Rho, Phys. Rep. 269, 333 (1996).

[12] R. Rapp and J. Wambach, Eur. Phys. J. A 6, 415 (1999).

[13] M. C. Abreu et al. [NA50 Collaboration], Eur. Phys. J. C 14 (2000) 443.

[14] R. Rapp and E. V. Shuryak, Phys. Lett. B 473, 13 (2000). 
[15] K. Gallmeister, O. P. Pavlenko and B. Kämpfer, Phys. Lett. B 473, 20 (2000).

[16] I. Kvasnikova, C. Gale and D. K. Srivastava Phys. Rev. C 65, 064903 (2002).

[17] M. M. Aggarwal et al. [WA98 Collaboration], Phys. Rev. Lett. 85, 3595 (2000).

[18] M. M. Aggarwal et al. [WA98 Collaboration], Phys. Rev. Lett. 93, 022301 (2004).

[19] S. Turbide, R. Rapp and C. Gale, Phys. Rev. C 69, 014903 (2004).

[20] D. K. Srivastava, Phys. Rev. C 71, 034905 (2005).

[21] W. Liu and R. Rapp, Nucl. Phys. A 796, 101 (2007).

[22] D. Adamova et al. [CERES Collaboration], Phys. Rev. Lett. 91, 042301 (2003).

[23] R. Shahoyan [NA60 Collaboration], J. Phys. G 34, S1029 (2007).

[24] F. Karsch and E. Laermann, arXiv:hep-lat/0305025.

[25] P. Braun-Munzinger, K. Redlich and J. Stachel, arXiv:nucl-th/0304013.

[26] F. Gelis, H. Niemi, P. V. Ruuskanen and S. S. Räsänen, J. Phys. G 30, S1031 (2004).

[27] B. Muller and K. Rajagopal, Eur. Phys. J. C 43, 15 (2005).

[28] D. d'Enterria and D. Peressounko, Eur. Phys. J. C 46, 451 (2006).

[29] R. Rapp and J. Wambach, Adv. Nucl. Phys. 25, 1 (2000).

[30] J. Alam, S. Sarkar, P. Roy, T. Hatsuda and B. Sinha, Ann. Phys. 286, 159 (2001).

[31] C. Gale and K. Haglin, arXiv:hep-ph/0306098.

[32] M. Harada and K. Yamawaki, Phys. Rep. 381, 1 (2003).

[33] G. E. Brown and M. Rho, Phys. Rep. 396, 1 (2004).

[34] M. Asakawa, T. Hatsuda and Y. Nakahara, Nucl. Phys. A 715, 863 (2003).

[35] E. V. Shuryak and I. Zahed, Phys. Rev. C 70, 021901 (2004).

[36] M. Mannarelli and R. Rapp, Phys. Rev. C 72, 064905 (2005).

[37] J. Casalderrey-Solana and E. V. Shuryak, arXiv:hep-ph/0408178.

[38] E. V. Shuryak and I. Zahed, arXiv:hep-ph/0406100.

[39] H. van Hees, V. Greco and R. Rapp, Phys. Rev. C 73, 034913 (2006).

[40] L. Grandchamp, R. Rapp and G. E. Brown, Phys. Rev. Lett. 92, 212301 (2004).

[41] F. Karsch, Lect. Notes Phys. 583, 209 (2002).

[42] E. L. Feinberg, Nuovo Cimento A 34, 391 (1976). 
[43] L. D. McLerran and T. Toimela, Phys. Rev. D 31, 545 (1985).

[44] R. Rapp, J. Phys. G 31, S217 (2005).

[45] F. Karsch, S. Datta, E. Laermann, P. Petreczky, S. Stickan and I. Wetzorke, Nucl. Phys. A 715, 701 (2003).

[46] E. Braaten, R. D. Pisarski and T. C. Yuan, Phys. Rev. Lett. 64, 2242 (1990).

[47] P. Petreczky, talk at the $2^{\text {nd }}$ RHIC-II meeting, Brookhaven National Laboratory (2005) http://www.phenix.bnl.gov/WWW/publish/david/rhicii_em/april2005/petreczky-RHIC2.ppt

[48] F. Arleo et al., CERN Yellow Report CERN-2004-009, (Geneva, Switzerland, 2004), arXiv:hep-ph/0311131.

[49] S. Gupta, Phys. Lett. B 597, 57 (2004).

[50] D. Fernandez-Fraile and A. Gomez-Nicola, Phys. Rev. D 73, 045025 (2006).

[51] C. R. Allton et al., Phys. Rev. D 71, 054508 (2005).

[52] M. Prakash, R. Rapp, J. Wambach and I. Zahed, Phys. Rev. C 65, 034906 (2002).

[53] D. Trnka et al. [CBELSA/TAPS Collaboration], Phys. Rev. Lett. 94, 192303 (2005)

[54] R. Rapp, J. Phys. G 34, S405 (2007).

[55] M. A. Shifman, A. I. Vainshtein and V. I. Zakharov, Nucl. Phys. B 147, 385 (1979).

[56] T. Hatsuda and S.-H. Lee, Phys. Rev. C 46, R34 (1992).

[57] M. Asakawa and C. M. Ko, Phys. Rev. C 48, 526 (1993).

[58] S. Leupold, W. Peters and U. Mosel, Nucl. Phys. A 628, 311 (1998).

[59] F. Klingl, N. Kaiser and W. Weise, Nucl. Phys. A 624, 527 (1997).

[60] J. Ruppert, T. Renk and B. Muller, Phys. Rev. C 73, 034907 (2006).

[61] S. Weinberg, Phys. Rev. Lett. 18, 507 (1967).

[62] T. Das, V. S. Mathur and S. Okubo, Phys. Rev. Lett. 19, 859 (1967).

[63] E. G. Floratos, S. Narison and E. de Rafael, Nucl. Phys. B 155, 115 (1979).

[64] S. Narison, Z. Phys. C 14, 263 (1982).

[65] V. Dmitrasinovic, Phys. Rev. D 57, 7019 (1998).

[66] J. I. Kapusta and E. V. Shuryak, Phys. Rev. D 49, 4694 (1994).

[67] R. Rapp, Phys. Rev. C 63, 054907 (2001). 
[68] R. Rapp, arXiv:nucl-th/0204003.

[69] P. Arnold, G. D. Moore and L. G. Yaffe, JHEP 0112, 009 (2001).

[70] S. Turbide, C. Gale, S. Jeon and G. D. Moore, Phys. Rev. C 72, 014906 (2005).

[71] D. Turbide, C. Gale, D. K. Srivastava and R. Fries, Phys. Rev. C 74 (2006) 014903.

[72] S. Bathe [PHENIX Collaboration], Nucl. Phys. A 774, 731 (2006).

[73] D. K. Srivastava and B. Sinha, Phys. Rev. C 64, 034902 (2001); D. K. Srivastava, Pramana 57, 235 (2001).

[74] J. e. Alam, S. Sarkar, T. Hatsuda, T. K. Nayak and B. Sinha, Phys. Rev. C 63, 021901 (2001).

[75] J. e. Alam, J. K. Nayak, P. Roy, A. K. Dutt-Mazumder and B. Sinha, J. Phys. G 34, 871 (2007).

[76] P. Huovinen, P. V. Ruuskanen and S. S. Räsänen, Phys. Lett. B 535, 109 (2002); S. S. Räsänen, Nucl. Phys. A 715, 717 (2003); H. Niemi, S. S. Räsänen and P. V. Ruuskanen CERN Yellow Report CERN-2004-009, arXiv:hep-ph/0311131.

[77] F. D. Steffen and M. H. Thoma, Phys. Lett. B 510, 98 (2001).

[78] M. Glück, E. Reya and A. Vogt, Phys. Rev. D 48, 116 (1993) [Erratum-ibid. D $\mathbf{5 1}, 1427$ (1995)].

[79] M. Gyulassy, I. Vitev, X.-N. Wang and B. W. Zhang, arXiv:nucl-th/0302077.

[80] F. Gelis, K. Kajantie and T. Lappi, Phys. Rev. Lett. 96, 032304 (2006).

[81] E. V. Shuryak and L. Xiong, Phys. Rev. Lett. 70, 2241 (1993).

[82] M. Strickland, Phys. Lett. B 331, 245 (1994).

[83] B. Kampfer, O. P. Pavlenko, A. Peshier and G. Soff, Phys. Rev. C 52, 2704 (1995)

[84] D. K. Srivastava, M. G. Mustafa and B. Muller, Phys. Rev. C 56, 1064 (1997).

[85] K. J. Eskola, B. Muller and X.-N. Wang, Phys. Lett. B 374, 20 (1996).

[86] D. M. Elliott and D. H. Rischke, Nucl. Phys. A 671, 583 (2000).

[87] T. Renk, S. A. Bass and D. K. Srivastava, Phys. Lett. B 632, 632 (2006).

[88] T. Renk, arXiv:hep-ph/0408218.

[89] S. A. Bass, B. Müller and D. K. Srivastava, Phys. Rev. Lett. 90, 082301 (2003).

[90] T. Sakaguchi [PHENIX Collaboration], Acta Phys. Hung. A 25, 409 (2006).

[91] S. Wicks, W. Horowitz, M. Djordjevic and M. Gyulassy, Nucl. Phys. A 784, 426 (2007). 
[92] G. Y. Qin, J. Ruppert, C. Gale, S. Jeon, G. D. Moore and M. G. Mustafa, arXiv:0710.0605 [hep-ph].

[93] R. Rapp, M. Urban, M. Buballa and J. Wambach, Phys. Lett. B 417, 1 (1998).

[94] J. V. Steele, H. Yamagishi and I. Zahed, Phys. Rev. D 56, 5605 (1997).

[95] R. Rapp, G. Chanfray and J. Wambach, Nucl. Phys. A 617, 472 (1997).

[96] M. Post, S. Leupold and U. Mosel, Nucl. Phys. A 689, 753 (2001).

[97] M.F.M. Lutz, G. Wolf and B. Friman, Nucl. Phys A 706, 431 (2002)

[98] D. Cabrera, E. Oset and M. J. Vicente-Vacas, Nucl. Phys. A 705, 90 (2002).

[99] V. L. Eletsky, M. Belkacem, P. J. Ellis and J. I. Kapusta, Phys. Rev. C 64, 035202 (2001).

[100] M. Urban, M. Buballa, R. Rapp and J. Wambach, Nucl. Phys. A 641, 433 (1998).

[101] R. Rapp and E. V. Shuryak, Phys. Rev. Lett. 86, 2980 (2001).

[102] T. Ishikawa et al., Phys. Lett. B 608, 215 (2005).

[103] R. Muto et al., Phys. Rev. Lett. 98, 042501 (2007).

[104] D. N. Voskresensky, Nucl. Phys. A 744, 378 (2004).

[105] R. Rapp, Nucl. Phys. A 661, 33 (1999).

[106] H. van Hees and R. Rapp, arXiv:0711.3444 [hep-ph].

[107] J. V. Steele and I. Zahed, Phys. Rev. D 60, 037502 (1999).

[108] G. E. Brown and M. Rho, Phys. Rev. Lett. 66, 2720 (1991); Phys. Rept. 269, 333 (1996).

[109] M. Harada and C. Sasaki, Phys. Rev. D 73, 036001 (2006).

[110] Y. Hidaka, O. Morimatsu and M. Ohtani, Phys. Rev. D 73, 036004 (2006).

[111] M. Harada and C. Sasaki, Phys. Rev. D 74, 114006 (2006).

[112] G. E. Brown, C. H. Lee and M. Rho, Nucl. Phys. A 747, 530 (2005).

[113] M. Velkovsky and E. V. Shuryak, Phys. Rev. D 56, 2766 (1997).

[114] G. E. Brown, C. H. Lee, M. Rho and E. V. Shuryak, Nucl. Phys. A 740, 171 (2004).

[115] M. Kitazawa, T. Kunihiro and Y. Nemoto, Phys. Lett. B 633, 269 (2006).

[116] R. Rapp, Phys. Rev. C 66, 017901 (2002).

[117] H. van Hees and R. Rapp, Phys. Rev. Lett. 97, 102301 (2006). 
[118] H. van Hees and R. Rapp, arXiv:hep-ph/0604269.

[119] T. Hirano and M. Gyulassy, Nucl. Phys. A 769, 71 (2006).

[120] S. A. Bass and A. Dumitru, Phys. Rev. C 61, 064909 (2000).

[121] D. Teaney, J. Lauret and E. V. Shuryak, Phys. Rev. Lett. 86, 4783 (2000).

[122] Z.-W. Lin, C.-M. Ko, B.-A. Li, B. Zhang and S. Pal, Phys. Rev. C 72, 064901 (2005).

[123] W. Cassing and S. Juchem, Nucl. Phys. A 672, 417 (2000).

[124] P. Huovinen an M. Prakash, Phys. Lett. B 450, 15 (1999).

[125] W. Cassing, E.L. Bratkovskaya, R. Rapp and J. Wambach, Phys. Rev. C 57, 916 (1997)

[126] T. Renk, R. Schneider, and W. Weise, Phys. Rev. C 66, 014902 (2002).

[127] R. Barate et al. [ALEPH Collaboration], Eur. Phys. J. C 4, 409 (1998).

[128] R. Rapp, Pramana 60, 675 (2003).

[129] R. Rapp, Nucl. Phys. A 725, 254 (2003).

[130] J. Adams et al. [STAR Collaboration], Phys. Rev. Lett. 92, 092301 (2004).

[131] M. Dey, V. L. Eletsky and B. L. Ioffe, Phys. Lett. B 252, 620 (1990).

[132] K. Dusling, D. Teaney and I. Zahed, Phys. Rev. C 75, 024908 (2007).

[133] J. Ruppert, C. Gale, T. Renk, P. Lichard and J. I. Kapusta, arXiv:0706.1934 [hep-ph].

[134] R. Chatterjee, D. K. Srivastava, U. W. Heinz and C. Gale, Phys. Rev. C 75, 054909 (2007).

[135] R. Arnaldi et al. [NA60 Collaboration], arXiv:0711.1816 [nucl-ex].

[136] T. Renk and J. Ruppert, arXiv:hep-ph/0612113.

[137] R. Rapp, H. van Hees and T. Strong, Braz. J. Phys. 37, 779 (2007) arXiv:hep-ph/0611339.

[138] E. Iancu and R. Venugopalan, arXiv:hep-ph/0303204;

J. Jalilian-Marian and Y.V. Kovchegov, Prog. Part. Nucl. Phys. 56, 104 (2006).

[139] F. Gelis and J. Jalilian-Marian, Phys. Rev. D 66, 014021 (2002); Phys. Rev.

D 66, 094014 (2002); Phys. Rev. D 67, 074019 (2003).

[140] B. Z. Kopeliovich, J. Raufeisen, A. V. Tarasov and M. B. Johnson, Phys. Rev. C 67, 014903 (2003);

M. B. Johnson et al., Phys. Rev. C 65, 025203 (2002).

[141] R. Baier, A. H. Mueller and D. Schiff, Nucl. Phys. A 741, 358 (2004);

M. A. Betemps and M. B. Gay Ducati, Phys. Rev. D 70, 116005 (2004). 
[142] J. Jalilian-Marian, Nucl. Phys. A 739, 319 (2004); Nucl. Phys. A 753, 307 (2005); Nucl. Phys. A 770, 210 (2006).

[143] A. Dumitru, A. Hayashigaki and J. Jalilian-Marian, Nucl. Phys. A 765, 464 (2006).

[144] R. D. Pisarski, Phys. Lett. B 100, 155 (1982).

[145] D. Miskowiec et al. [CERES Collaboration], Nucl. Phys. A 774 , 43 (2006).

[146] S. Afanasiev et al., arXiv:0706.3034v1 [nucl-ex].

[147] E. V. Shuryak and G. E. Brown, Nucl. Phys. A 717, 322 (2003).

[148] W. Broniowski, W. Florkowski and B. Hiller, Phys. Rev. C 68, 034911 (2003).

[149] S. Pratt and W. Bauer, Phys. Rev. C 68, 064905 (2003).

[150] P. Fachini, J. Phys. G 30, S735 (2004).

[151] L. Holt and K. Haglin, J. Phys. G 31, S245 (1995);

S. Pal, C. M. Ko and Z. Lin, Nucl. Phys. A 707, 525 (2002);

L. Alvarez-Ruso, V. Koch, Phys. Rev. C 65, 054901 (2002).

[152] A. Kozlov et al. [PHENIX Collaboration], Nucl. Phys. A 774, 739 (2006).

[153] X. Cai, et al. [STAR Collaboration], Nucl. Phys. A 774, 485 (2006) 485;

J. Adams et al. [STAR Collaboration], Phys. Lett. B 612, 181 (2005).

[154] J. Rafelski, J. Letessier, G. Torrieri, Phys. Rev. C 72, 024905 (2005).

[155] D. Adamova et al. [CERES Collaboration], Phys. Rev. Lett. 96, 152301 (2006).

[156] E. Scomparin et al. [NA60 Collaboration], Nucl. Phys. A 774, 67 (2006).

[157] S. Afanasiev et al. [PHENIX Collaboration], Phys. Rev. Lett. 99, 052301 (2007).

[158] A. Adare et al. [PHENIX Collaboration], Phys. Rev. D 76, 051106 (2007).

[159] T. Isobe [PHENIX Collaboration], J. Phys. G 34, S1015 (2007).

[160] F. Arleo, JHEP 0609, 015 (2006).

[161] S. Turbide, C. Gale and R. J. Fries, Phys. Rev. Lett. 96, 032303 (2006).

[162] R. Chatterjee, E. S. Frodermann, U. W. Heinz and D. K. Srivastava, Phys.

Rev. Lett. 96, 202302 (2006).

[163] S. S. Adler et al. [PHENIX Collaboration], Phys. Rev. Lett. 96, 032302 (2006).

[164] S. S. Adler et al. [PHENIX Collaboration], Phys. Rev. Lett. 96, 032301 (2006).

[165] A. Adare et al. [PHENIX Collaboration], Phys. Rev. Lett. 98, 172301 (2007).

[166] B. I. Abelev et al. [STAR Collaboration], Phys. Rev. Lett. 98, 192301 (2007). 
[167] E. Shuryak, Phys. Rev. C 55, 961 (1997).

[168] Yu. L. Dokshitzer, D. E. Kharzeev, Phys. Lett. B 519, 199 (2001).

[169] N. M. Kroll and W. Wada, Phys. Rev. 98, 1355 (1955).

[170] M. Shao et al. Nucl. Instrum. Meth. A 558, 419 (2006).

[171] Z. Xu et al. LBNL-PUB-5509, "A heavy flavor tracker for STAR", http://www-library.lbl.gov/docs/PUB/5509/PDF/PUB-5509.pdf;

K. Schweda et al., Nucl. Phys. A 774, 907 (2006).

[172] Z. Frankel et al., Nucl. Instrum. Meth. A 546, 466 (2005).

[173] H. Satz, J. Phys. G 32, R25 (2006).

[174] R. Rapp, D. Cabrera and H. van Hees, arXiv:nucl-th/0608033.

[175] S. A. Bass, B. Müller and D. K. Srivastava, Phys. Rev. Lett. 93, 162301 (2004).

[176] J. Sandweiss et al., in preparation.

[177] M. A. Lisa, S. Pratt, R. Soltz, U. Wiedemann, Ann. Rev. Nucl. Part. Sci. 55, 357 (2005).

[178] R. Rapp, Eur. Phys. J. A 18, 459 (2003).

[179] J. Adams et al. [STAR Collaboration], Phys. Rev. Lett. 92, 092301 (2004).

[180] P. Fachini, private communication (2006).

[181] S. Vogel and M. Bleicher, arXiv:hep-ph/0607242.

[182] J. Adams et al. [STAR Collaboration], Phys. Rev. C 70, 044902 (2004). 